\begin{tabular}{|c|c|c|c|}
\hline $\begin{array}{l}\text { 1. Identification Number: } \\
\text { DE-FG26-0NT08854 }\end{array}$ & $\begin{array}{l}\text { 2. Program } / \mathrm{Pr} \\
\text { Nanopart } \\
\text { Non-Fooc }\end{array}$ & $\begin{array}{l}\text { ct Title: } \\
\text { le Technology } \\
\text { Cource Feedsto }\end{array}$ & $\begin{array}{l}\text { or Biorefining of } \\
\text { ks }\end{array}$ \\
\hline $\begin{array}{l}\text { 3. Recipient: } \\
\text { Ames Laboratory }\end{array}$ & & & \\
\hline 4. Reporting Requirements: & Frequency & No. of Copies & Addressees \\
\hline $\begin{array}{l}\text { A. MANAGEMENT REPORTING } \\
\text { 冈 Progress Report } \\
\bigotimes \text { Special Status Report }\end{array}$ & Q & $\begin{array}{l}\text { Upload only } 1 \text { copy } \\
\text { to the address in the } \\
\text { next column at the } \\
\text { interval specified in } \\
\text { the previous column. }\end{array}$ & $\begin{array}{l}\text { https://www.ere- } \\
\text { pmc.energy.gov/SubmitReports.aspx }\end{array}$ \\
\hline $\begin{array}{l}\text { B. SCIENTIFIC/TECHNICAL REPORTING } \\
\text { (Reports/Products must be submitted with appropriate DOE F 241. The } \\
241 \text { forms are available at www.osti.gov/elink) }\end{array}$ & & & \\
\hline $\begin{array}{lc}\quad \text { Report/Product } & \text { Form } \\
\text { Q Final Scientific/Technical Report } & \text { DOE F 241.3 } \\
\square \text { Conference papers/proceedings* } & \text { DOE F 241.3 } \\
\square \text { Software/Manual } & \text { DOE F 241.4 } \\
\square \text { Other (see Special Instructions) } & \text { DOE F 241.3 } \\
{ }^{*} \text { Scientific and technical conferences only } & \end{array}$ & & & $\begin{array}{l}\text { http://www.osti.gov/elink-2413 } \\
\text { http://www.osti.gov/elink-2413 } \\
\text { http://www.osti.gov/estsc/241-4pre.jsp }\end{array}$ \\
\hline $\begin{array}{l}\text { C. FINANCIAL REPORTING } \\
\text { \SF-269, Financial Status Report } \\
\square \text { SF-269A, Financial Status Report (Short Form) } \\
\square \text { SF-272, Federal Cash Transactions Report }\end{array}$ & & & $\begin{array}{l}\text { https:///www.ere- } \\
\text { pmc.energy.gov/SubmitReports.aspx }\end{array}$ \\
\hline $\begin{array}{l}\text { D. CLOSEOUT REPORTING } \\
\otimes \text { Patent Certification } \\
\square \text { Property Certification } \\
\square \text { Other (see Special Instructions) }\end{array}$ & & & $\begin{array}{l}\text { https:///www.ere- } \\
\text { pmc.energy.gov/SubmitReports.aspx }\end{array}$ \\
\hline $\begin{array}{l}\text { E. OTHER REPORTING } \\
\bigotimes \text { Annual Indirect Cost Proposal } \\
\bigotimes \text { Annual Inventory Report of Federally Owned Property, if any } \\
\square \text { Other }\end{array}$ & $\begin{array}{l}\mathrm{A} \\
\mathrm{A}\end{array}$ & & $\begin{array}{l}\text { https://www.eere- } \\
\text { pmc.energy.gov/SubmitReports.aspx }\end{array}$ \\
\hline FREQUENCY CODES AND DUE DATES: & & & \\
\hline $\begin{array}{l}\text { A - Within } 5 \text { calendar days after events or as specified. } \\
\text { F - Final; } 90 \text { calendar days after expiration or } \\
\text { termination of the award. } \\
\text { Y - Yearly; } 90 \text { days after the end of the reporting } \\
\text { period. }\end{array}$ & $\begin{array}{l}S-\mathrm{Se} \\
\text { reportir } \\
\text { Q- Quart }\end{array}$ & $\begin{array}{l}\text { iannually; with } \\
\text { period. } \\
\text { y; within } 30 \text { days afte }\end{array}$ & 30 days after end of \\
\hline 5. Special Instructions: Forms are available at https://www.eere-pmc.energ & /forms.asp. & & \\
\hline
\end{tabular}


Nanoparticle Technology for Biorefinery of Non-Food Source Feedstocks

Award Number:

DE-FG26-0NT08854

Final Technical Progress Report

Reporting Period:

May 30, 2008 to September 30, 2012

Submitted By:

lowa State University

1138 Pearson Hall

Ames, IA 50011-2207

Principal Investigator:

Dr. Marek Pruski

Senior Scientist, U.S. DOE Ames Laboratory

Adjunct Professor, Department of Chemistry

lowa State University

230 Spedding Hall

Ames, IA 50011-3111

515-294-2017 / 515-294-4709 (fax)

mpruski@iastate.edu

Date Report Issued:

January 22, 2013 


\section{DISCLAIMER}

This report was prepared as an account of work sponsored by an agency of the United States Government. Neither the United States Government nor any agency thereof, nor any of their employees, makes any warranty, express of implied, or assumes any legal liability or responsibility for the accuracy, completeness, or usefulness of any information, apparatus, product, or process disclosed, or represents that its use would not infringe privately owned rights. Reference herein to any specific commercial product, process, or service by trade name, trademark, manufacturer, or otherwise does not necessarily constitute or imply its endorsement, recommendation, or favoring by the United States Government or any agency thereof. The views and opinions of authors expressed herein do not necessarily state or reflect those of the United States Government or any agency thereof. 
DE-FG26-0NT08854

\title{
Nanoparticle Technology for Biorefinery of Non-Food Source Feedstocks
}

\author{
Dr. Marek Pruski \\ Dr. Brian G. Trewyn \\ Dr. Young-Jin Lee \\ Dr. Victor S.-Y. Lin \\ Ames Laboratory \\ lowa State University
}

\begin{abstract}
The goal of this proposed work is to develop and optimize the synthesis of mesoporous nanoparticle materials that are able to selectively sequester fatty acids from hexane extracts from algae, and to catalyze their transformation, as well as waste oils, into biodiesel. The project involves studies of the interactions between the functionalized MSN surface and the sequestering molecules. We investigate the mechanisms of selective extraction of fatty acids and conversion of triglycerides and fatty acids into biodiesel by the produced nanoparticles. This knowledge is used to further improve the properties of the mesoporous nanoparticle materials for both tasks. Furthermore, we investigate the strategies for scaling the synthesis of the catalytic nanomaterials up from the current pilot plant scale to industrial level, such that the biodiesel obtained with this technology can successfully compete with food crop-based biodiesel and petroleum diesel.
\end{abstract}

Keywords: nanocatalysts, biodiesel, fatty acids, mesoporous materials, heterogeneous catalysis, material characterization, solid-state NMR, mass spectrometry 


\section{TABLE OF CONTENTS}

Disclaimer

. i

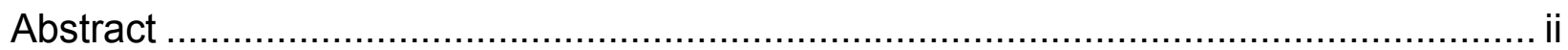

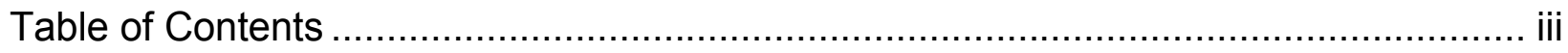

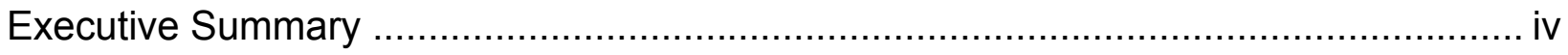

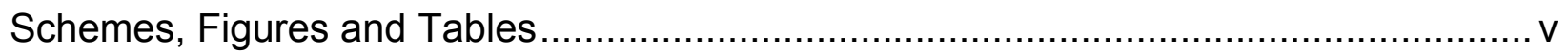

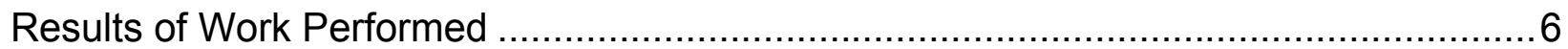

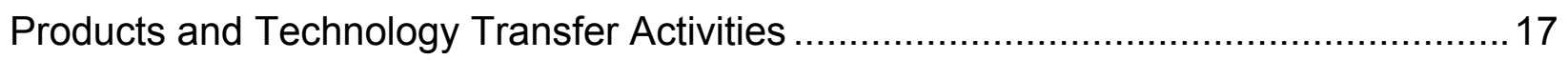

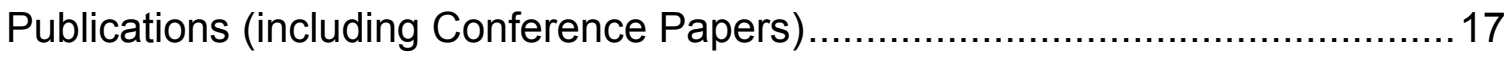

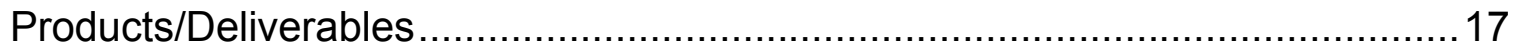

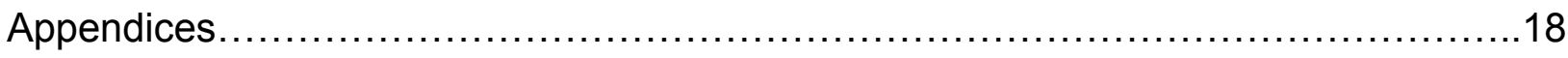




\section{EXECUTIVE SUMMARY}

The objective is to develop a process that will selectively sequester fatty acids from hexane extracts from algae and to catalyze their transformation to biodiesel. This effort involved close collaboration between scientists at lowa State University, Ames Laboratory, and scientists and engineers at Catilin, which became AlbemarleCatilin during the reporting period.

This investigation was divided into two phases. Phase 1 was the synthesis and characterization of mesoporous silica nanoparticles (MSN) and mesoporous carbon nanoparticles (MCN) followed by quantifying the capture and sequestration of free fatty acids from mixed hydrocarbon solutions. Phase 2 was the continued characterization and improvement of MSN-based heterogeneous catalysts for conversion of nonfood feedstocks to biodiesel, and their implementation by Catilin, Inc. into pilot plant scale operation.

This report is a summary of the work and the final results that we achieved. Complete results of our studies have been submitted in quarterly reports and can be found in the appendices in forms of published papers, a thesis chapter and intellectual property disclosure. 


\section{SCHEMES, FIGURES, AND TABLES}

Figure 1. TEM images of ordered mesoporous a) $M S N$ and b) MCN samples.

Figure 2. The effect of functionalization on the capacity for FFA sequestration.

Figure 3. Quantitative solid-state ${ }^{29}$ Si NMR spectra of a) MSN functionalized with ionic liquid by co-condensation and b) MSN functionalized with ionic liquid by grafting. $\mathrm{T}^{2}$ and $\mathrm{T}^{3}$ sites represent $\mathrm{Si}$ atoms directly bound to carbon.

Figure 4. ${ }^{29} \mathrm{Si}$ solid-state NMR spectra of MSNs functionalized with aminopropyltrimethoxysilane for (a) 6 hours and (b) 12 hours at reflux temperature in methanol.

Figure 5. MALDI mass spectrum of simulated algae oil solution with a known concentration of $200 \mathrm{mM}$ for each fatty acid and the internal standard, $\mathrm{C}_{19}$.

Figure 6. Orbitrap MS spectra of algal crude oils ionized with ESI, APCI, APPI, and MALDI. Most ions are detected as protonated, except potatiation of TAG and DAG in ESI. Chlorophyll $a$ is detected as [chlorophyll a- $\left.\mathrm{Mg}^{2+}+2 \mathrm{H}\right]^{+}(\mathrm{ChIA})$ or as an oxidation product, [chlorophyll $\left.a+O-\mathrm{Mg}^{2+}+2 \mathrm{H}\right]^{+}\left(\mathrm{ChIA}^{*}\right)$. Sterols are detected with a water-loss, $\left[\mathrm{M}-\mathrm{H}_{2} \mathrm{O}+\mathrm{H}\right]^{+}$, labeled as $\mathrm{C}$, E, and St for cholesterol, ergosterol, and stigmasterol, respectively. Squalene and $\alpha-$ tocopherol are labeled as $\mathrm{Sq}$ and $\mathrm{T}$, respectively, and chlorophyll $\mathrm{a}$ fragments are labeled as ChIAF. $\alpha$-tocopherol is detected as a protonated ion in $\mathrm{APCl},[\mathrm{M}+\mathrm{H}]^{+}$, and as a radical ion in $\mathrm{APPI}, \mathrm{M}^{+\cdot}$.

Figure 7. Scanning electron micrograph (a) and transmission electron micrograph (b) of nonfunctionalized LPMSN.

Figure 8. SEM images of SA-SBA-15 (a) and SA-LPMSN (b). Scale bar $=2 \mu \mathrm{m}$ and 3 $\mu \mathrm{m}$, respectively.

Figure 9. Kinetic studies of the esterification reaction catalyzed by SA-LPMSN-100C (black) and SA-SBA15 (red).

Figure 10. X-ray diffraction (XRD) spectra of mesoporous calcia silicate catalysts for transesterification reactions: MCS- $1-1.0 \mathrm{~g} \mathrm{CaO}, \mathrm{MCS}-2-0.5 \mathrm{~g} \mathrm{CaO}$, and MCS-3 - $0.25 \mathrm{~g} \mathrm{CaO}$.

Table 1. Semi-quantitative comparison of each algal lipid after various nanoparticle treatment

Table 2. Quantification of surface acid sites on a series of MCS catalytic materials via ammonia TPD analysis. 
DE-FG26-0NT08854

\section{RESULTS OF WORK PERFORMED}

Heterogeneous catalysts have been developed for the conversion of non-food source feedstocks to biodiesel. The specific objectives of this project were to develop an array of porous heterogeneous catalysts to selectively adsorb free fatty acids (FFAs), and to efficiently catalyze their conversion into biodiesel. To this end, we have made significant research progress on two major tasks: (1) identify the most favorable surface functional group and pore size of mesoporous nanoparticle material for optimal sequestration of FFAs, and (2) determine optimal catalyst properties and reaction conditions for the conversion of nonfood feedstocks to biodiesel. In this final report, we describe the research challenges and progress in the synthesis of mesoporous silica and carbon based nanomaterials, along with efficiency and selectivity results toward sequestration of FFAs. The synthetic effort is accompanied by extensive material characterizations. We are gratified with the accomplishments of this project, and believe that our results will provide a solid foundation for the future development of heterogeneous catalysts for the conversion of nonfood-based feedstocks to biodiesel and for addressing the challenges that come with commercialization of this process. Appendices 1-4 include three publications, a thesis chapter (to be published); an intellectual property disclosure resulting from this project has not been included in this report.

\section{Material development}

\subsection{Synthesis and characterization of mesoporous materials for selective sequestration of free fatty acids (FFAs).}

We began our research activities by optimizing the synthesis of mesoporous silica nanoparticle (MSN) materials for the selective sequestration of FFAs. The detailed synthesis of the FFA sequestering MSN can be found in the paper referenced in Appendix 1. Utilizing the high surface area, tunable pore size, and ability to functionalize the MSN surface, we synthesized a series of these materials by varying the synthesis conditions and additives to optimize the capture and sequestration of FFAs. Once the synthesis of MSNs is well established and understood, these parameters can be varied with relative ease to yield the desired product.

\section{Synthesis of mesoporous silica nanoparticle (MSN) materials:}

Typically, $480 \mathrm{ml}$ aqueous solution of cetyltrimethylammonium bromide (CTAB, $2.0 \mathrm{~g}$, $5.5 \mathrm{mmol}), \mathrm{NaOH}(7.0 \mathrm{~mL} \times 2.0 \mathrm{M}, 14.0 \mathrm{mmol})$ was heated to $80{ }^{\circ} \mathrm{C}$ and the hydrolysis of tetraethylorthosilicate (TEOS, $10.0 \mathrm{~mL}, 44.8 \mathrm{mmol}$ ) occurred over $2 \mathrm{~h}$ at $80{ }^{\circ} \mathrm{C}$. After the hydrolysis of TEOS, the white solid was filtrated and dried under vacuum overnight. This MSN material was either calcined at $600{ }^{\circ} \mathrm{C}$ for $5 \mathrm{~h}$ (when no organic surface functional groups were incorporated) or heated in an acidic methanol solution (when surface organic moieties were included) to remove the pore templating surfactant. We performed a complete material characterization of each MSN material using transmission electron microscopy (TEM), scanning electron microscopy (SEM), low 
angle powder X-ray diffraction (XRD), and nitrogen sorption analysis prior to and, in most cases, after testing the sequestration capacity of the individual MSN. All these analytical techniques were available in Dr. Victor Lin/Dr. Brian Trewyn laboratory. Surface functional groups are incorporated into the MSN materials by two methods: 1) post-synthesis grafting and 2) in situ co-condensation. Incorporation of the organic moieties onto the surface of MSN via post synthesis grafting involves refluxing the functional group (i.e. 3-aminopropyltrimethoxysilane) in toluene with a suspension of MSN for $12 \mathrm{~h}$. Functionalization via co-condensation involves mixing the functional group with the silica monomer (TEOS) during the hydrolysis and condensation step at $80{ }^{\circ} \mathrm{C}$ (Figure 1a). Additionally, various synthetic conditions can be adjusted to control the particle morphology, pore size, and specific surface area; the detailed synthetic method reported here is a common procedure for MSN.

\section{Synthesis of mesoporous carbon nanoparticle (MCN) materials:}

Mesoporous carbon nanoparticle (MCN) materials were synthesized using a mesoporous aluminosilicate hard-template procedure. The MSN was converted to mesoporous aluminosilicate by adding a solution of $\mathrm{AlCl}_{3}$ to the material. Specifically, the calcined MSN was mixed with distilled water to form silanol groups on the surface of the material, and then completely dried at $150^{\circ} \mathrm{C}$. The dried sample was suspended in an ethanol solution of anhydrous $\mathrm{AlCl}_{3}(\mathrm{Si} / \mathrm{Al}=20)$. The ethanol solvent was removed under reduced pressure. The dried sample was calcined at $550{ }^{\circ} \mathrm{C}$ to produce the final mesoporous aluminosilicate template.

Ordered MCN materials were prepared using furfuryl alcohol as the carbon source. Mesoporous aluminosilicate nanoparticles $(1 \mathrm{~g})$ were infiltrated with $0.91 \mathrm{~mL}$ of furfuryl alcohol by an impregnation method. The mixture was kept under vacuum at $35^{\circ} \mathrm{C}$ for 1 h. The mixture was heated for $6 \mathrm{~h}$ at $100^{\circ} \mathrm{C}$ for polymerization of furfuryl alcohol and partially carbonized at $350{ }^{\circ} \mathrm{C}$ for $3 \mathrm{~h}$ under vacuum. After cooling to room temperature, the sample was charged with an additional $0.58 \mathrm{~mL}$ of furfuryl alcohol, and repeated freezevacuum-thaw and polymerization. Further
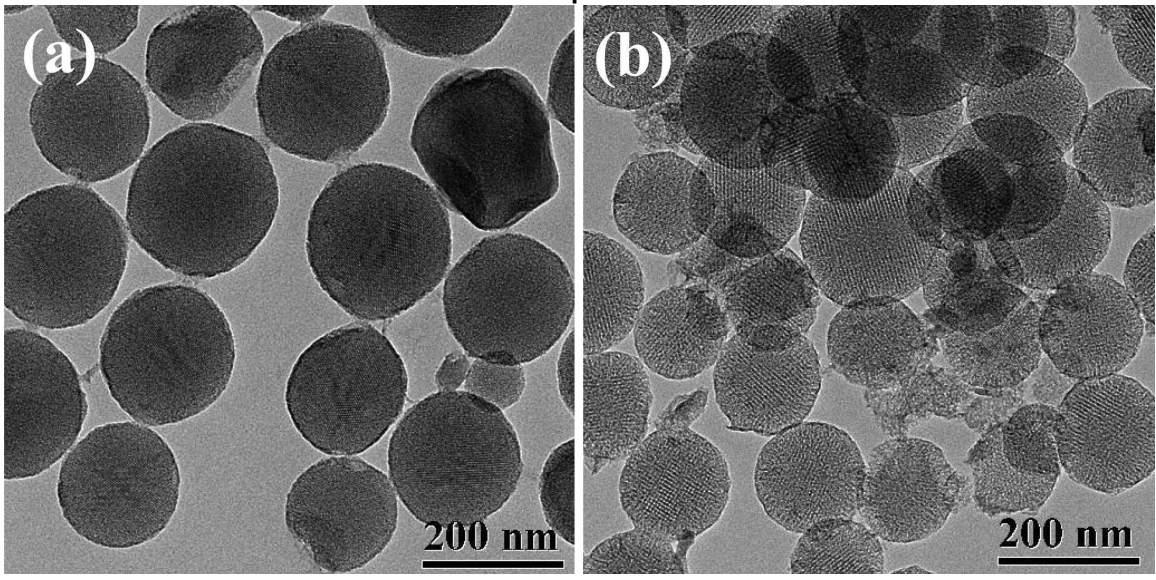

Figure 1. TEM images of ordered mesoporous a) MSN and b) MCN samples.

carbonization was accomplished by heating to $900{ }^{\circ} \mathrm{C}$ under vacuum conditions. The pure MCN product (Figure 1b) was collected by removing the aluminosilicate template via treatment with $\mathrm{HF}\left(10 \mathrm{wt} \% \mathrm{HF}\right.$ in $\mathrm{EtOH} / \mathrm{H}_{2} \mathrm{O}$ solution). 
Through the sequestration studies, we determined that silica was superior to carbon as our core material for the synthesis of the mesoporous nanoparticle materials.

\section{Characterization and optimization of pore size and surface functionalization of MSN:}

\section{The effect of MSN Pore Size on FFA Sequestration:}

Initially, two MSN materials with different pore sizes were synthesized and tested to determine the dependence of FFA sequestration on pore diameter. Mesoporous silica nanoparticle materials with a pore diameter of 2.5-nm (MSN-2.5) were synthesized following the synthesis procedure described above and MSNs with 10-nm pores (MSN10) were synthesized by using the procedure reported in Appendix 1. The FFA sequestration capacity of MSN-2.5 and MSN-10 were 0.40 and 0.65 mmol FFA g ${ }^{-1}$, respectively. Since MSN-10 was shown to sequester $63 \%$ more FFAs, it was chosen as the base material for continued studies.

\section{Optimizing surface functional group for FFA sequestration via MSN-10:}

In another series of experiments, we used the post-synthesis grafting method to incorporate eight different functional groups into the MSNs with $10 \mathrm{~nm}$ pores (MSN-10), and analyzed their capacity for FFA capture and sequestration. The functional groups included:

aminoethyl)3-

aminopropyl

aminopropyl

$\mathrm{N}-(2-$ benzenesulfonic acid, $\beta$ cyclodextrin, hexadecyl, nonfunctional, ionic liquid $(\mathrm{PMImCl})$ and thiol. The GC-MS results (see Figure 2) show that the AP (aminopropyl) functional group was most efficient for sequestering the FFAs. Indeed, under the conditions used in our measurements, nearly $98 \%$ of the FFA in the simulated solution was

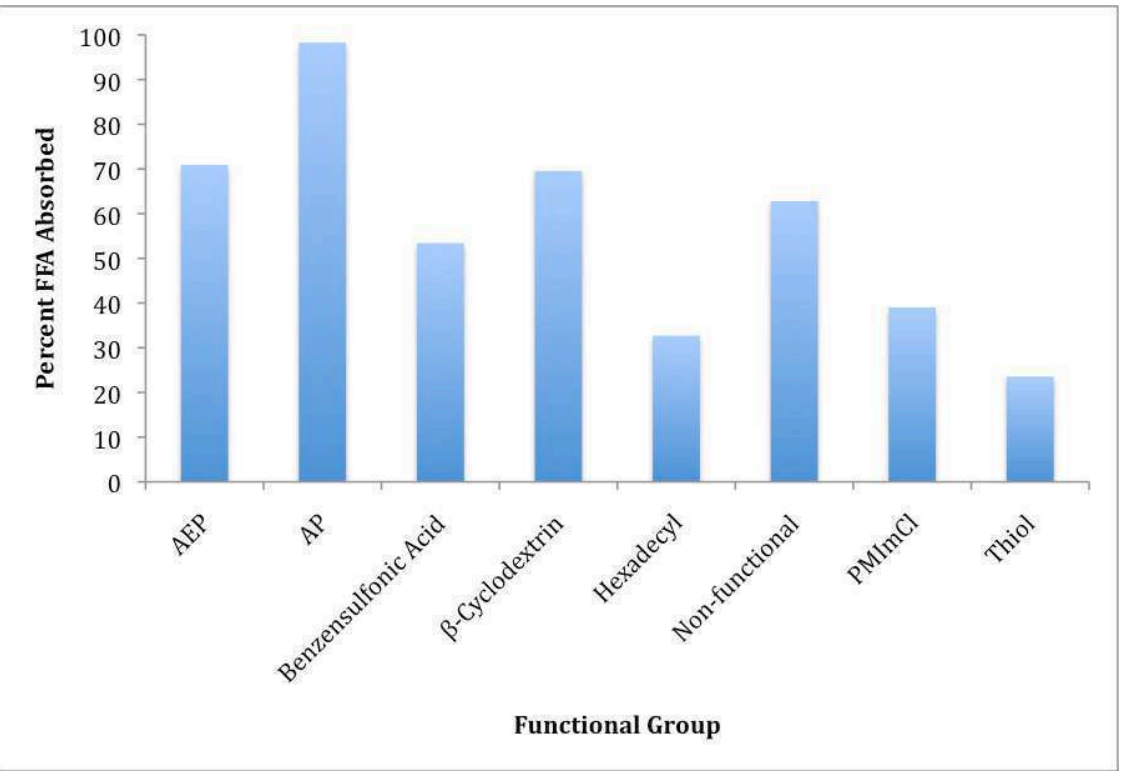

Figure 2. The effect of functionalization on the capacity for FFA sequestration. adsorbed by the MSN10 functionalized with this amine. Based on the findings of this study, we selected the AP functional group on MSN-10 to target the removal of FFAs from biofuel feedstocks.

The characterizations of the functionalized MSNs were carried out using powder X-ray diffraction, TEM, SEM, nitrogen adsorption/desorption and TGA analysis. In addition, we used extensive solid-state NMR characterization, in the laboratory of Dr. Marek Pruski. Without going into specifics, we have determined, through ${ }^{13} \mathrm{C}$ and ${ }^{29} \mathrm{Si}$ solid-state NMR, that the desired functional groups were indeed bound to the surface, and that the 
efficiency of surface functionalization strongly depends on the method of synthesis (grafting vs co-condensation, see Figure 3). These results demonstrated the advantage of post-synthesis grafting approach, which was consequently used in further studies.

a)

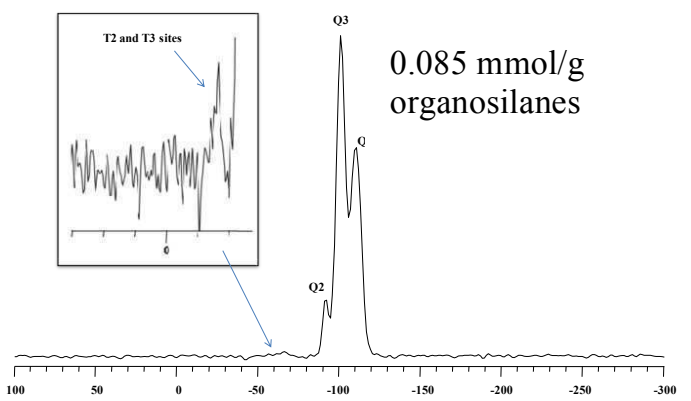

b)

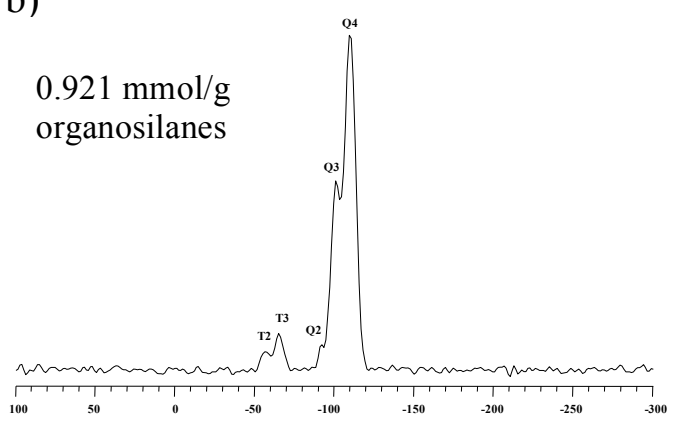

Figure 3. Quantitative solid-state ${ }^{29} \mathrm{Si}$ NMR spectra of a) MSN functionalized with ionic liquid by co-condensation and b) MSN functionalized with ionic liquid by grafting. $\mathrm{T}^{2}$ and $\mathrm{T}^{3}$ sites represent $\mathrm{Si}$ atoms directly bound to carbon.

To further improve the materials' performance, Dr. Pruski's group analyzed the efficiency of functionalization by solid-state NMR. The integration of ${ }^{29} \mathrm{Si}$ NMR resonances representing the carbon-bound T-sites in a series of samples indicated that 12-hour reaction times yielded optimal coverage of grafted AP functional groups (Figure 4). In addition to investigating the reaction times, and after consultations with our industrial partner, we also examined different reaction solvents, specifically toluene, ethanol, and methanol. We determined that as the reflexing temperature of the solvent increased, so did the amount of the MSN-bound functional groups (i.e. toluene resulted in the greatest amount of MSN-bound functional groups).

(a)

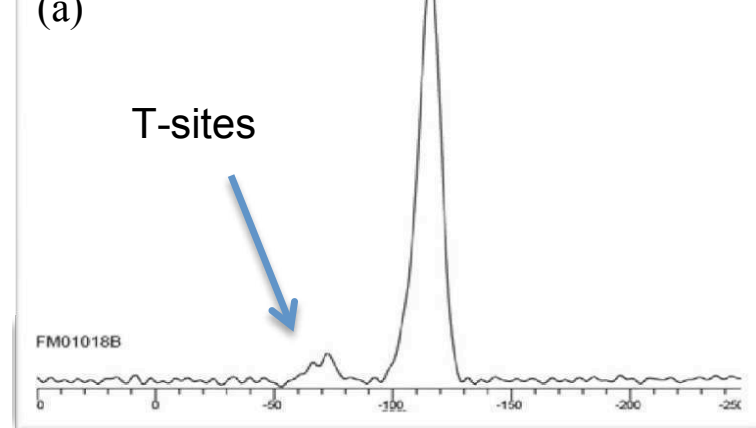

(b)

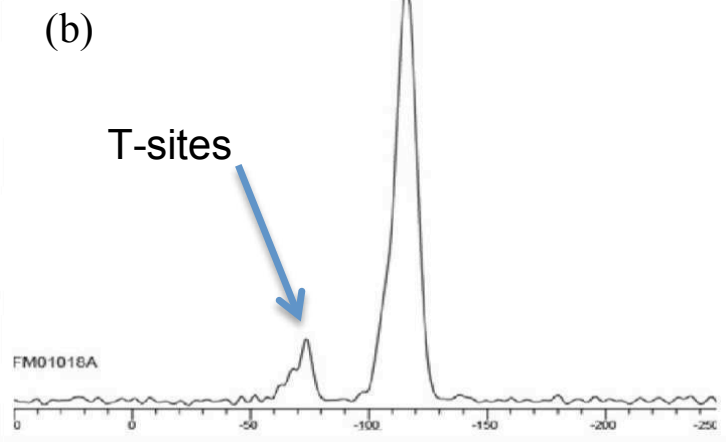

Figure 4. ${ }^{29} \mathrm{Si}$ solid-state NMR spectra of MSNs functionalized with aminopropyltrimethoxysilane for (a) 6 hours and (b) 12 hours at reflux temperature in methanol.

Figures 1-4 exemplify the synthesis and characterization strategies used in our quest to maximize fatty acid uptake and sequestration. More detailed descriptions of results that resulted from these efforts can be found in the papers referenced in the appendices. As part of this investigation, we carried out fundamental solid-state NMR investigations of the conformations of MSN-bound functional groups in the presence of solvents. This 
study, which is detailed in Appendix 2, recently led to the discovery of a very efficient catalyst for the esterification reaction.

\section{Mass spectrometry analysis of algal oil and sequestered bioproducts:}

The synthesis, characterization and optimization of MSNs were followed by quantitative studies of FFA sequestration. Dr. Young-Jin Lee's research group analyzed the algae oils by MALDI-MS, initially using their previously established GC-MS protocol. Figure 5, below, illustrates some of the initial results from MALDI-MS of simulated algal oil after adsorption, sequestration, and subsequent removal from nonfunctionalized MSN to

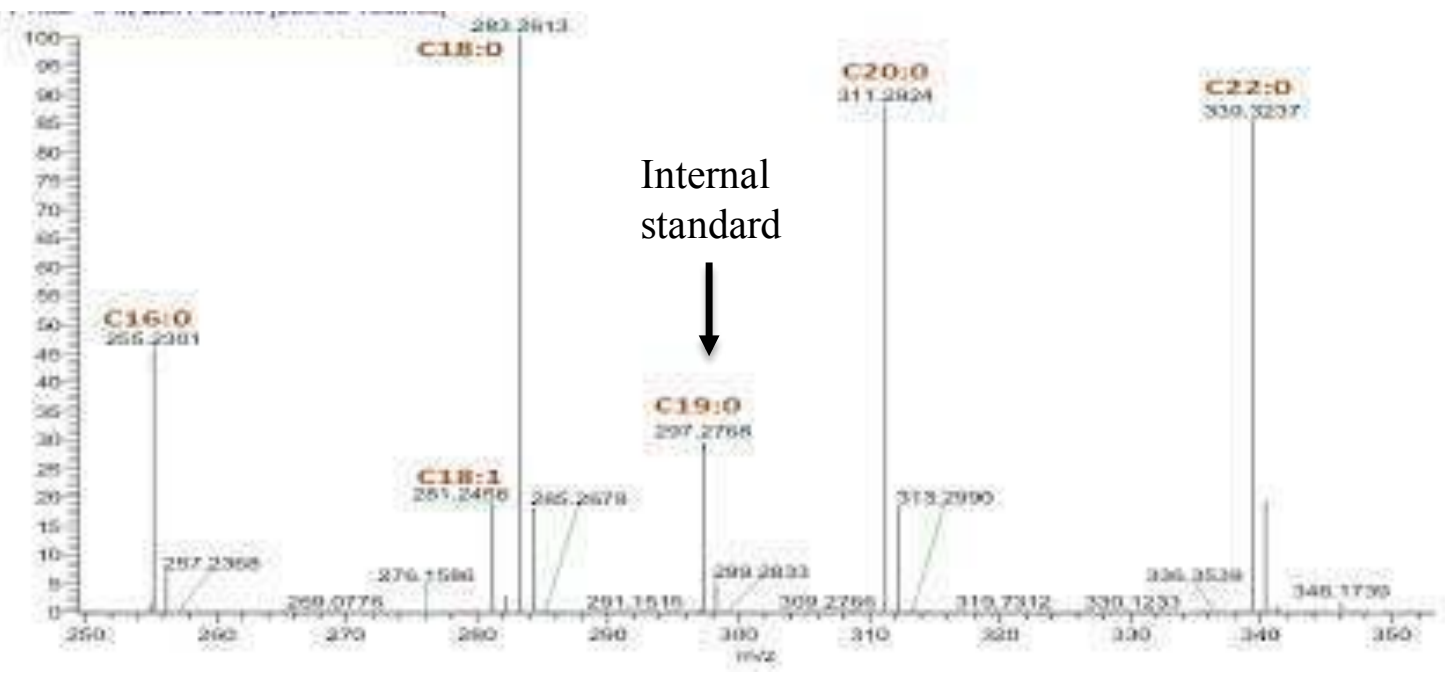

Figure 5. MALDI mass spectrum of simulated algae oil solution with a known concentration of $200 \mathrm{mM}$ for each fatty acid and the internal standard, C19.

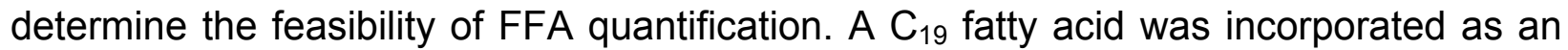
internal standard because algae do not produce this fatty acid. We discovered that all fatty acids of interest in the algae oil have similar ionization efficiencies; however, in the standard MALDI mode the vapor pressure of the FFAs caused inconsistencies with quantification. Due to these results achieved early in this study, Dr. Lee's group was able to develop protocols for high-throughput assay of naturally produced molecules from microalgae.

\section{High-throughput assay for monitoring non-volatile lipids of algal oil:}

Although the GC-MS based method has been well-proven in many applications and is being used in hundreds of laboratories, this approach has a few limitations for other compounds in that 1) it needs multiple sample preparation steps that may affect quantification reliability, 2) the derivatization step, to make them volatile and protected from reaction with column stationary phase, is often problematic, 3) some non-volatile major compounds, such as TAG (triacylglycerides), could not be analyzed with GC-MS, and 4) the whole process takes too long, not appropriate for the high-throughput analysis. In this regard, the Lee group has developed a high-throughput assay for nonvolatile compound analysis using high-resolution mass spectrometry, which will be more suitable for algae extracts. 


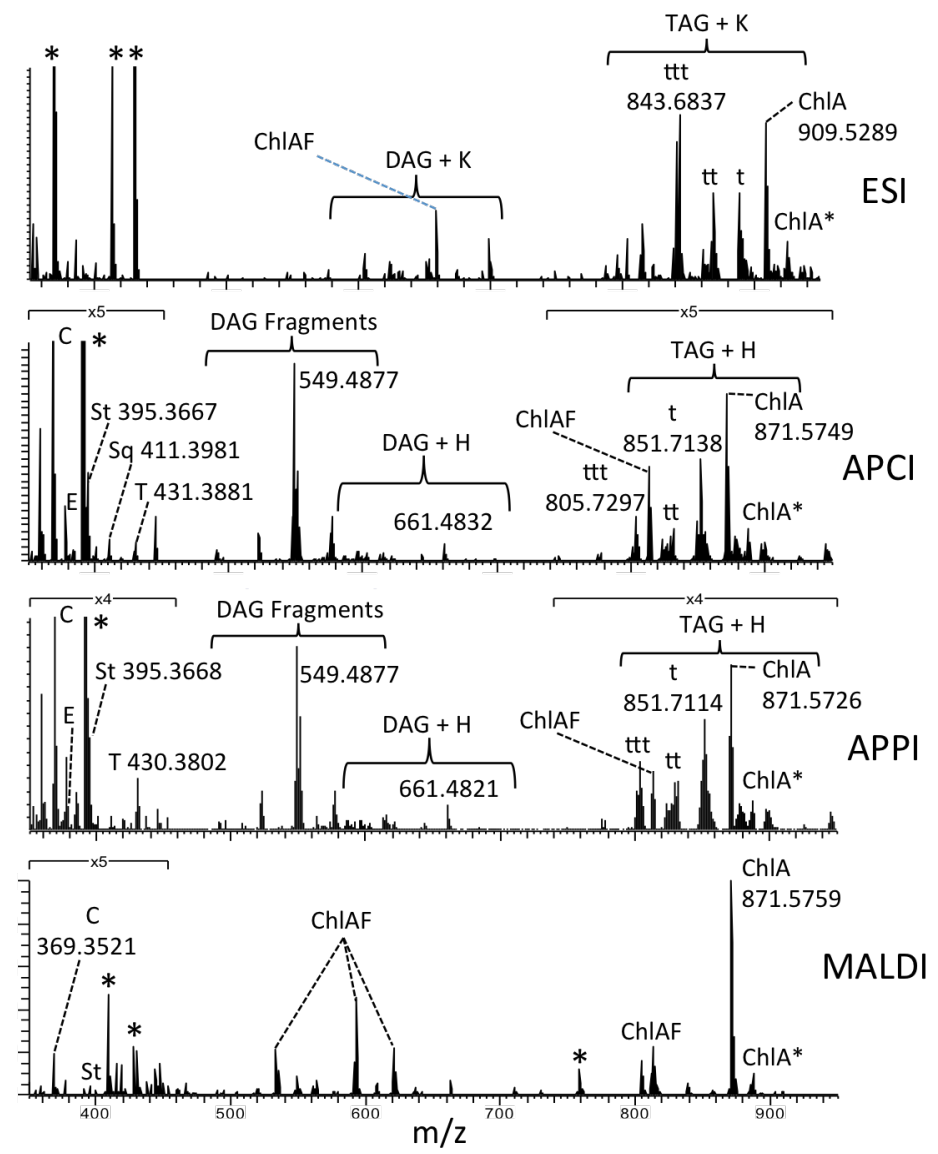

Figure 6. Orbitrap MS spectra of algal crude oils ionized with ESI, APCI, APPI, and MALDI. Most ions are detected as protonated, except potatiation of TAG and DAG in ESI. Chlorophyll $a$ is detected as [chlorophyll a$\left.\mathrm{Mg}^{2+}+2 \mathrm{H}\right]^{+} \quad$ (ChIA) or as an oxidation product, [chlorophyll $\left.\mathrm{a}+\mathrm{O}-\mathrm{Mg}^{2+}+2 \mathrm{H}\right]^{+}\left(\mathrm{ChIA}^{*}\right)$. Sterols are detected with a water-loss, $\left[\mathrm{M}-\mathrm{H}_{2} \mathrm{O}+\mathrm{H}\right]^{+}$, labeled as $\mathrm{C}, \mathrm{E}$, and St for cholesterol, ergosterol, and stigmasterol, respectively. Squalene and a-tocopherol are labeled as Sq and T, respectively, and chlorophyll a fragments are labeled as ChIAF. $\alpha$-tocopherol is detected as a protonated ion in $\mathrm{APCl},[\mathrm{M}+\mathrm{H}]^{+}$, and as a radical ion in APPI, $\mathrm{M}^{+}$.

Table 1. Semi-quantitative comparison of each algal lipid after various nanoparticle treatment.

\begin{tabular}{|c|c|c|c|c|c|}
\cline { 2 - 6 } \multicolumn{1}{c|}{} & Control & AP-MSN-3 & AP-MSN-5 & AP-MSN-10 & MSN-10 \\
\hline TAG & $100(8.2)$ & $103(13.5)$ & $106(12.7)$ & $117(14.3)$ & $122(11.9)$ \\
\hline DAG & $100(8.6)$ & $28(12.0)$ & $79(14.2)$ & $59(13.7)$ & $35(14.3)$ \\
\hline Cholesterol & $100(8.0)$ & $75(10.0)$ & $96(11.8)$ & $100(16.5)$ & $94(11.7)$ \\
\hline Ergosterol & $100(3.3)$ & $58(14.5)$ & $88(14.7)$ & $85(15.6)$ & $74(13.5)$ \\
\hline Stigmasterol & $100(2.9)$ & $84(4.4)$ & $102(13.1)$ & $101(3.1)$ & $93(4.8)$ \\
\hline Squalene & $100(72.6)$ & $56(55.8)$ & $79(35.6)$ & $70(43.1)$ & $74(33.4)$ \\
\hline a-tocopherol & $100(4.0)$ & $89(13.2)$ & $94(12.9)$ & $106(11.7)$ & $111(22.5)$ \\
\hline Chlorophyll A & $100(2.5)$ & $18(27.3)$ & $85(11.2)$ & $85(8.0)$ & $22(22.5)$ \\
\hline
\end{tabular}

Note: TAG and DAG are sum of ion intensities of all TAG and DAG compounds; relative quantification was made in normalization to the corresponding ion signals in the control; standard deviation is given in parenthesis. 
An initial step of this study is to find the most appropriate ionization method. As shown in Figure 6 obtained for algal crude oils from Solarix, electrospray ionization (ESI) ionizes only polar compounds, i.e., TAG, DAG, and chlorophyll a. In MALDI, chlorophyll suppresses most of the compounds including TAG. However, APPI and APCl could effectively ionize most of the lipids of interest, including TAG, DAG, chlorophyll a, sterols, and terpenes. Between the two, APCl was chosen because of the better sensitivity, especially for squalene. When $\mathrm{APCl}$ was used with orbitrap high-resolution mass spectrometer, total of 48 lipid species (32 TAG, 10 DAG, 3 sterols, squalene, $\alpha-$ tocopherol, chlorophyll a) could be characterized in less than one-minute of data acquisition time. This assay could also be successfully demonstrated in application to mesoporous nanoparticle treatment of algal crude oils, enabling to study its effect on sequestration of various lipid species (Table 1). Further details can be found in the recently published paper on these MS advances (Appendix 3).

\section{Synthesis, characterization, and catalytic esterification activity of LPMSNs functionalized with sulfonic acid:}

Another important aspect of this study, in addition to the development of FFA sequestration nanotechnology, was to investigate novel heterogeneous catalysts for biodiesel applications. As part of this effort, we developed an original method for the cocondensed synthesis of MSNs with high surface area, large pore size (9-12 nm), and uniform morphology. The synthesis utilized a triblock copolymer as the pore templating agent, dissolved in a low $\mathrm{pH}$ aqueous acid solution along with tetramethylorthosilicate (TMOS), followed by surface functionalization with e.g., thiol groups. The full description of synthesis and characterization can be found in Appendix 4. The SEM and TEM micrographs of the nonfunctional large-pore MSN (LPMSN) are shown in Figure 7.
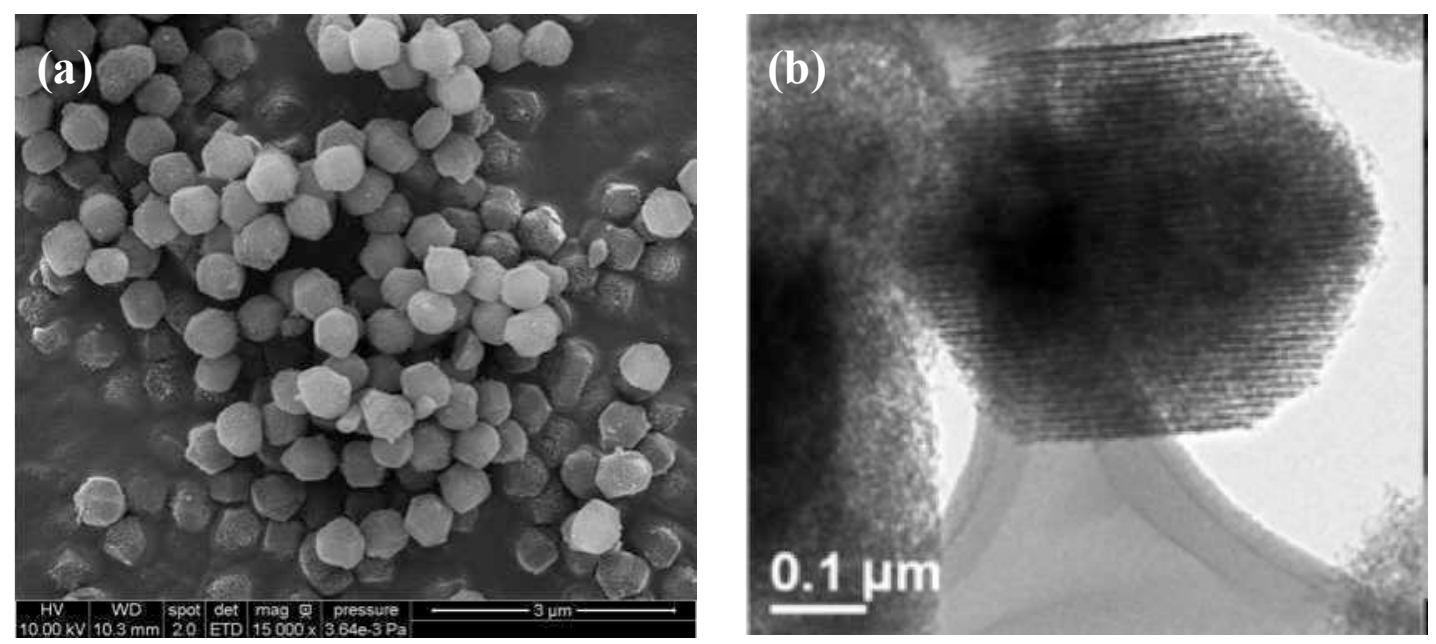

Figure 7. Scanning electron micrograph (a) and transmission electron micrograph (b) of nonfunctionalized LPMSN.

Once we demonstrated the ability to control the morphology of the nonfunctional LPMSNs, we focused on functionalizing the surface with organic acids for potential applications in esterification. Figure 8 shows the SEM images of previously published 

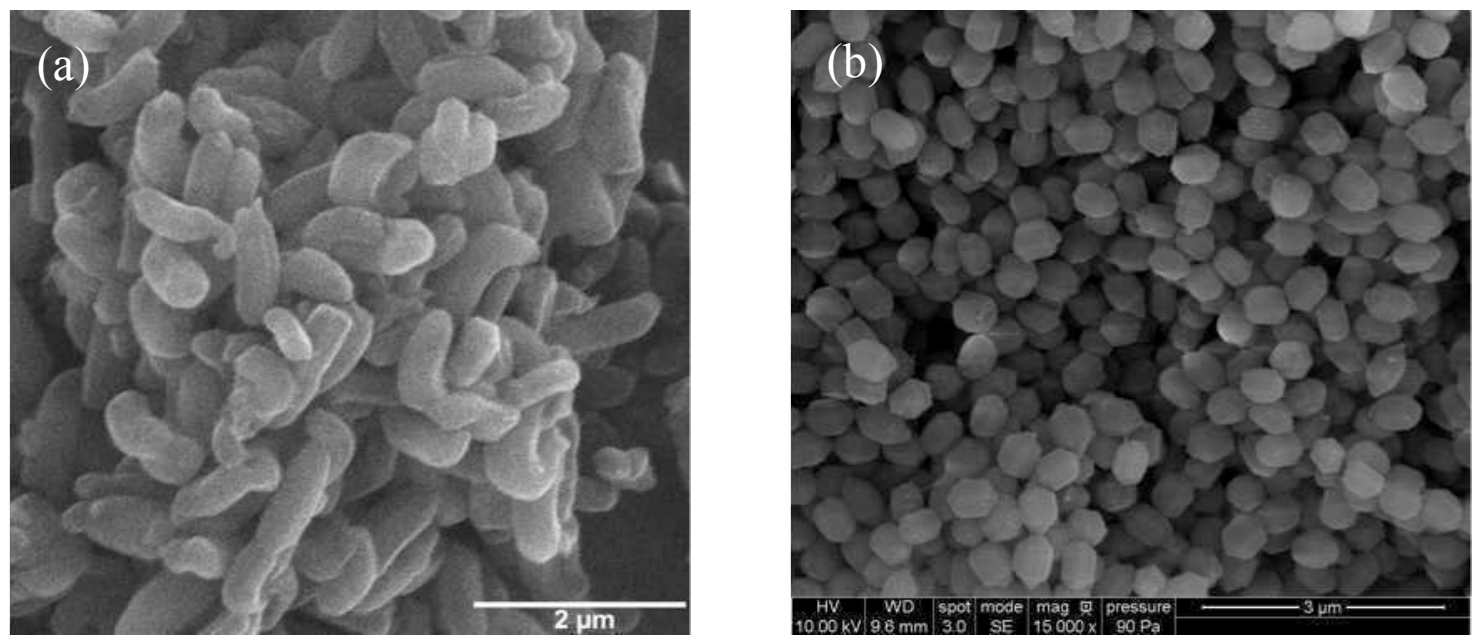

Figure 8. SEM images of SA-SBA-15 (a) and SA-LPMSN (b). Scale bar $=$ $2 \mu \mathrm{m}$ and $3 \mu \mathrm{m}$, respectively.

porous heterogeneous sulfonic acid catalysts (SA-SBA-15, (a)) and the sulfonic acid catalysts synthesized in our laboratory (SA-LPMSN-100, (b)). All characterization data and results can be found in Appendix 4. We tested the catalytic activity of SALPMSN for esterification of FFA to fatty acid methyl ester (FAME) and compared it to the catalytic esterification of SA-SBA-15. As can be seen in Figure 9, the catalyst we developed outperformed its predecessor. This project also involved extensive characterization of the surface bound functional groups by solid-state NMR. The description of solid-state

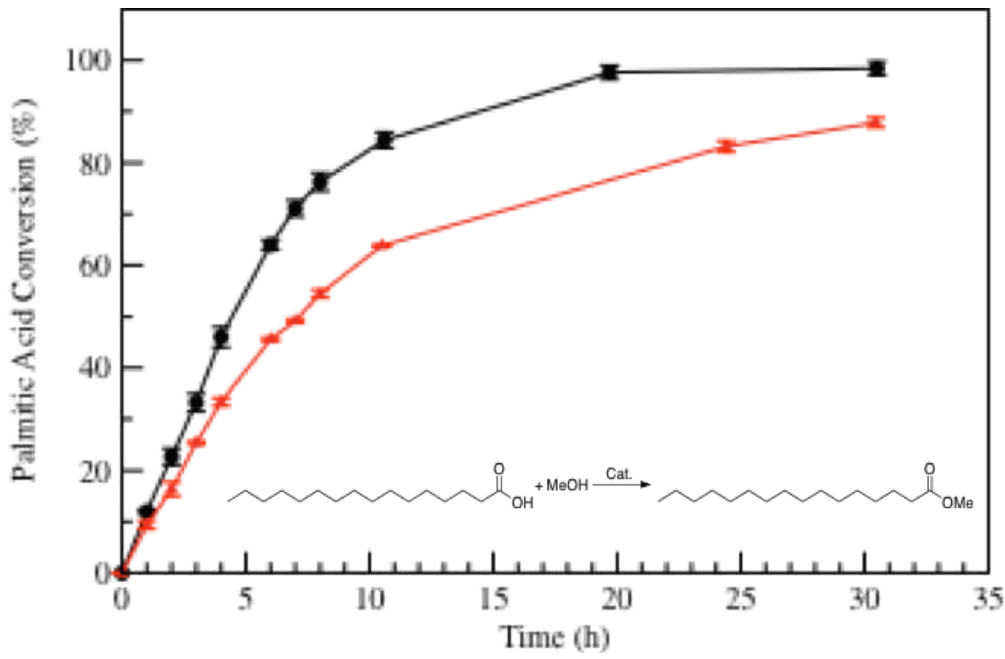

Figure 9. Kinetic studies of the esterification reaction catalyzed by SA-LPMSN-100 ${ }^{\circ} \mathrm{C}$ (black) and SA-SBA-15 (red). NMR analyses can be found in Appendices 2 and 4 .

\section{Characterization of Catilin's heterogeneous mesoporous calcia silicate (MCS) biodiesel catalyst:}

Although the characterization of MCS materials was advanced upon submitting the original research proposal, one important property of these catalysts was investigated as part of this grant. We set out to correlate the quantity of acid sites on the surface of MCS with the measured activity for conversion of triacylglycerides to FAMEs (biodiesel). We anticipated that measuring the concentration of acid sites could afford quick and reliable screening of the best transesterification/esterification catalysts. Utilizing a newly 
acquired chemisorption analyzer, we used gaseous ammonia to quantify the number of acid sites on the surface. In addition to quantifying the surface acid sites, we were able to measure the relative acid strength. In Table 2, the measured surface acid sites are reported. Each sample is unique by the amount of $\mathrm{CaO}$ that is added to the synthesis of the catalyst; specifically, MCS- 1 used $1 \mathrm{~g}$ of $\mathrm{CaO}$, MCS-2 used $0.5 \mathrm{~g}$ of $\mathrm{CaO}$, and MCS3 used $0.25 \mathrm{~g} \mathrm{CaO}$. In addition to quantifying the number and strength of the surface acid sites, we studied the chemical nature of the MCS transesterification catalysts. We
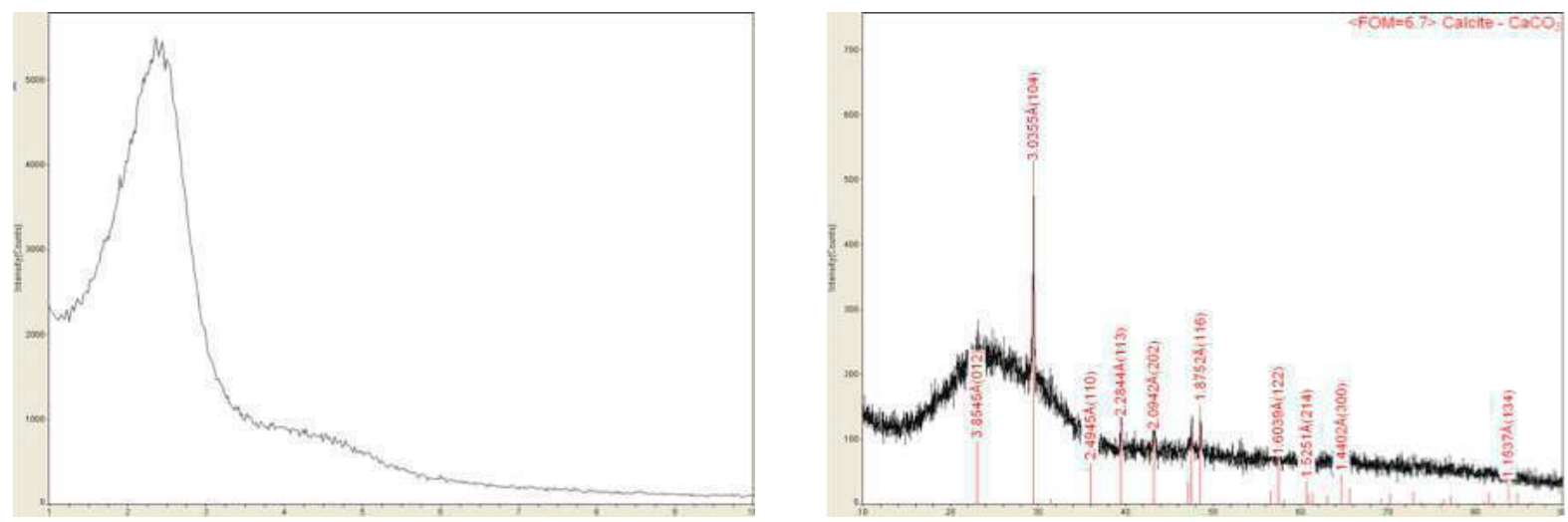

Calcined MCS-3
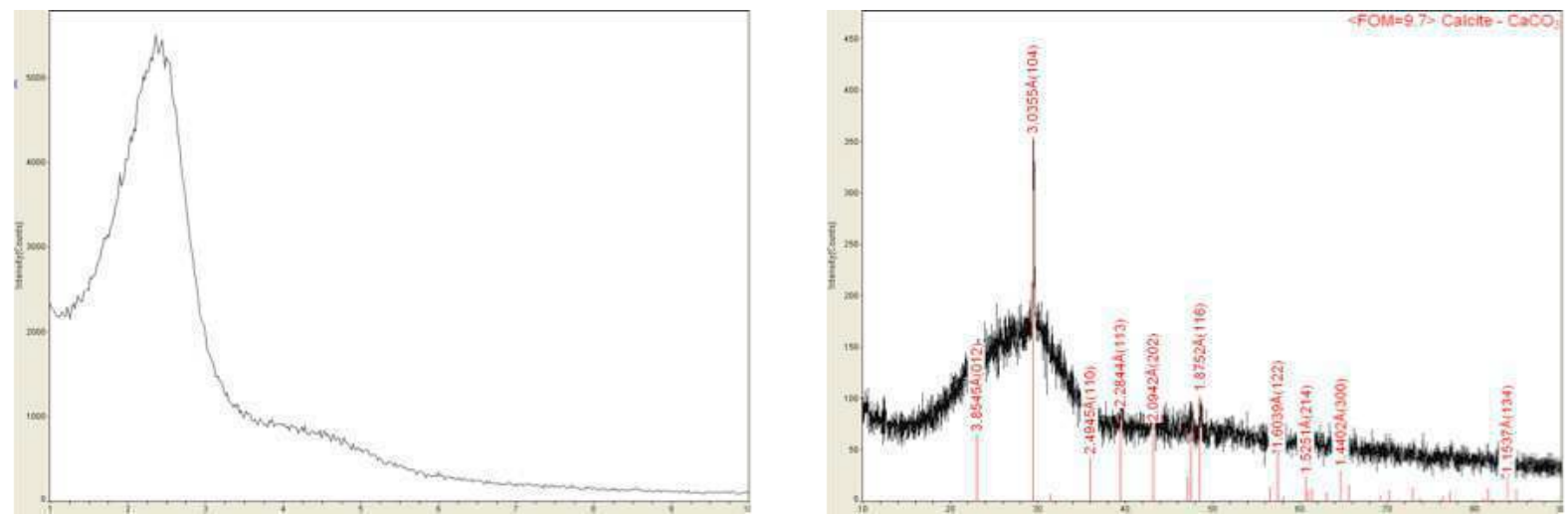

Calcined MCS-2
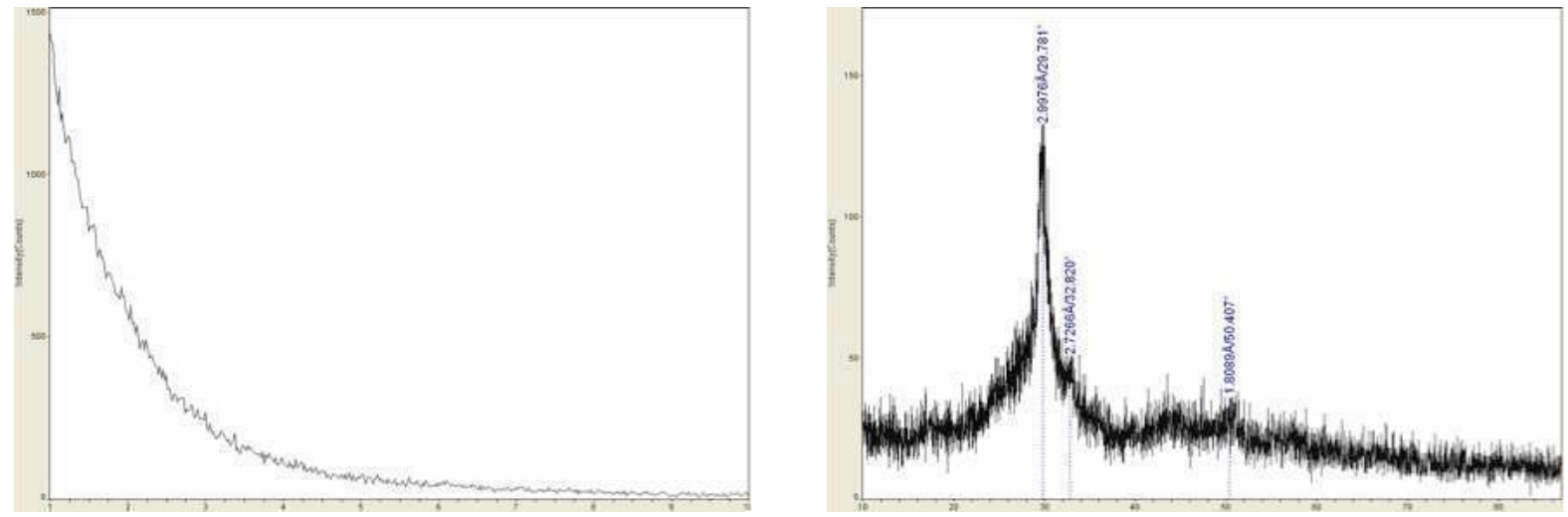

Calcined MCS-1

Figure 10. X-ray diffraction (XRD) spectra of mesoporous calcia silicate catalysts for transesterification reactions: MCS-1 - $1.0 \mathrm{~g} \mathrm{CaO}, \mathrm{MCS}-2-0.5 \mathrm{~g} \mathrm{CaO}$, and MCS-3 - $0.25 \mathrm{~g} \mathrm{CaO}$. 
have made several low and high angle $X$ ray diffraction (XRD) measurements of three different catalysts synthesized with different amounts of $\mathrm{Ca}$ (Figure 10). The $\mathrm{XRD}$ analysis revealed the presence of calcite (calcium carbonate) in the catalysts with decreased amounts of calcium. Development of surface chemisorption analysis for these catalysts has yet to come to fruition but we have made progress in understanding their surface chemistry.

\section{Development, commercialization and pilot plant maturation of heterogeneous catalyst by Catilin:}

At the onset of this project, Catilin was a small company with approximately eight employees working at a pilot-scale biodiesel plant with a 1 million gallon per year capacity at the Biomass Energy Conversion (BECON) facility of the lowa Energy Center, Nevada, lowa. This pilot plant was converted from an existing traditional homogeneous base catalyzed transesterification plant to one that utilizes a heterogeneous mesoporous calcia silicate catalyst, first in a batch mode and then as a continuous flow plant. The conversion from batch to continuous flow operation took the better part of the first year of this study. Following the successful demonstration of new technology, Catilin spent a significant amount of effort searching for biodiesel producers that utilize homogeneous catalysts to convert their systems to Catilin's heterogeneous technology. In the beginning, Catilin produced MCS catalyst in $0.5 \mathrm{~kg}$ batches with the goal of developing methods to produce catalyst in batches of at least $5 \mathrm{~kg}$. Subsequently, Catilin identified and implemented another catalyst, proprietarily named T300, which is an inexpensive byproduct of an existing industry and had higher activity and much lower capital cost than MCS. Because of this development, Catilin shifted their focus toward understanding the properties of T300. The partnership with Catilin was highly valuable to us, as we developed our sequestration nanotechnology, especially in identifying potential areas of cost reduction for large-scale production of the most promising MSN materials. Shortly after the sudden death of Dr. Victor Lin (Catilin founder), the managing group and coowner of Catilin, Mohr Davidow Ventures, began the search for opportunities of merging Catilin with an existing corporation to continue further development and commercialization of the catalyst. Nearly a year after the passing of Dr. Lin and extensive rearrangement of the technical and management personnel, Catilin was acquired by Albemarle Corp. to form Albemarle Catilin. Albemarle Catilin continues to operate the pilot plant in Nevada, IA, while actively pursuing customers for their proprietary catalyst technology. The local employment grew to over 20 employees in the four years from the inception of this study. 


\section{Conclusions:}

As a result of this project we gained considerable knowledge about the design, synthesis, and characterization of MSN materials for sequestration and catalytic transformation of extracts from algae into biodiesel. This investigation has resulted in three peer-reviewed publications, one thesis chapter (to be published), and one intellectual property document. This final report summarizes the results of synergistic collaboration between several research groups at the Ames Laboratory, lowa State University, and Catilin, Inc. Specifically, material synthesis and characterization were carried out by the Victor Lin/Brian Trewyn research group, while Marek Pruski's solidstate NMR research provided atomic-level understanding of the structure and functioning of these materials. Dr. Lee's group made significant advances in analyzing these oils by MS techniques, by overcoming severe limitations of the previously established GC-MS methods. Finally, having an industrial partner in this study gave us invaluable guidance for the synthesis of most useful materials at the lowest cost and in a safe manner. The strong collaborative relationships, specifically among the groups at ISU and Ames Laboratory developed during this study will continue to serve us well in the future.

In summary, we determined that optimal performance in sequestration was achieved in MSN materials with $10 \mathrm{~nm}$ pore diameter, functionalized with primary amines via a postsynthesis grafting method. The best method to identify the chemically different molecules produced by algae utilizes various mass spectrometry techniques strongly dependent on physical properties of the individual molecules. Finally, our industrial partner identified a commercially available product that showed improved heterogeneous transesterification activity over the original MCS type catalyst. 
DE-FG26-0NT08854

\section{PRODUCTS AND TECHNOLOGY TRANSFER ACTIVITIES}

\section{Publications (including Conference Papers)}

1. Justin S. Valenstein, Kapil Kandel, Forrest Melcher, Igor I. Slowing, Victor S.-Y. Lin, and Brian G. Trewyn, "Functional Mesoporous Silica Nanoparticles for the Selective Sequestration of Free Fatty Acids from Microalgal Oil", ACS Appl. Mater. Interfaces, 2012, 4, 1003-1009. (Appendix 1)

2. Kanmi Mao, Takeshi Kobayashi, Jerzy W. Wiench, Hung-Ting Chen, ChihHsiang Tsai, Victor S.-Y. Lin, and Marek Pruski, "Conformations of Silica-Bound (Pentafluorophenyl)propyl Groups Determined by Solid-State NMR Spectroscopy and Theoretical Calculations", J. Am. Chem. Soc., 2010, 132, 12452-12457. (Appendix 2)

3. Young Jin Lee, Rachael C. Leverence, Erica A. Smith, Justin S. Valenstein, Kapil Kandel, and Brian G. Trewyn, "High-Throughput Analysis of Algal Crude Oils Using High Resolution Mass Spectrometry", Lipids, 2013, DOI 10.1007/s11745-013-3757-7. (Appendix 3)

4. Wei Huang, Chih-Hsiang Tsai, Takeshi Kobayashi, Marek Pruski, Hung-Ting Chen, ${ }^{*}$ Brain G. Trewyn, ${ }^{*}$ and Victor S.-Y. Lin, "Organic functionalized mesoporous silica nanoparticles with large pore diameters as efficient heterogeneous catalysts", Wei Huang's Ph.D. thesis chapter, to be published. (Appendix 4)

\section{Products/Deliverables}

Intellectual Property Disclosure to the Office of Intellectual Property and Technology Transfer and the lowa State University Research Foundation submitted on "Magnetic Mesoporous Material for the Accumulation and Removal of Microalgae Cells from Concentrated and Dilute Microalgae Cultures."

\section{REFERENCES}

See references in Appendix 1. 


\title{
Functional Mesoporous Silica Nanoparticles for the Selective Sequestration of Free Fatty Acids from Microalgal Oil
}

\author{
Justin S. Valenstein, Kapil Kandel, Forrest Melcher, Igor I. Slowing, Victor S.-Y. Lin, ${ }^{\dagger}$ \\ and Brian G. Trewyn*
}

Department of Chemistry and U.S. Department of Energy-Ames Laboratory, Iowa State University, Ames, Iowa 50011, United States

ABSTRACT: A series of 2d-hexagonally packed mesoporous silica nanoparticle material with $10 \mathrm{~nm}$ pore diameter (MSN-10) covalently functionalized with organic surface modifiers have been synthesized via a post-synthesis grafting method. The material structure has been characterized by powder X-ray diffraction, electron microscopy, and nitrogen sorption analyses, and the free fatty acid (FFA) sequestration capacity and selectivity was investigated and quantified by thermogravimetric and GC/MS analysis. We discovered that aminopropyl functionalized $10 \mathrm{~nm}$ pore mesoporous silica nanoparticle material (AP-MSN-10) sequestered all available FFAs and left nearly all other molecules in solution from a simulated microalgal extract containing FFAs, sterols, terpenes, and triacylglycerides. We also demonstrated selective FFA sequestration from commercially available microalgal oil.

KEYWORDS: mesoporous silica, free fatty acids, selective sequestration, microalgal oil

\section{INTRODUCTION}

The U.S. and the European Union produce nearly 1.5 billion liters of biodiesel annually. ${ }^{1}$ The high cost of biodiesel can be greatly attributed to the high costs of the oil feedstock and are slowing the global commercialization. One solution is to use waste oils ${ }^{2,3}$ and nonfood sources ${ }^{4}$ as feedstocks. Algae present themselves as a potential feedstock source because many species grow in oceanwater or wastewater and produce more energy than other biofuel crops. ${ }^{5}$ The large-scale production of biodiesel was recently demonstrated by $\mathrm{Li}$ and co-workers when they published the requirements to successfully grow algae to a density of $14.2 \mathrm{~g} \mathrm{~L}^{-1}$ in an $11000 \mathrm{~L}$ bioreactor with a lipid content of $44.3 \%$ dry weight and converted the oil to fatty acid methyl esters (FAMEs or biodiesel) through transesterification using immobilized lipase. ${ }^{6}$

Because algae are known to produce a wide variety of highvalue and value-added hydrocarbons and lipids, ${ }^{7,8}$ it is necessary to develop technology to separate the algae-produced molecules to optimize the economics of converting algae oil to energy. An example of a high-value hydrocarbon found in algae is free fatty acids (FFAs), where some have considerable value to the pharmaceutical, neutraceutical, and cosmetic industries, particularly the omega-3 and other polyunsaturated FFAs. Another reason to selectively sequester FFAs from feedstocks is the negative effects they have on the conversion of oils to biodiesel. Biodiesel is currently produced from very low FFA percentage $(<1 \%)$ crop oils by the conversion of triacylglycerides (TAGs) through a base-catalyzed transesterification. When the concentration of acid in the feedstock is too high, as they are often found in algal oil, the basic catalyst is neutralized and additional catalyst is required to maintain the reaction kinetics.

Current separation and purification methods for organic acids are challenging and energy intensive, with extraction and distillation the most common techniques, either using organic solvents or supercritical fluids. ${ }^{9-11}$

The discovery and development of surfactant micelle-templated mesoporous silica nanoparticle (MSN) materials with high surface area and pore volume have advanced the utilization of these materials for applications in catalysis, ${ }^{12,13}$ sensors, ${ }^{14}$ delivery vessels, ${ }^{15-20}$ and adsorbents. ${ }^{21}$ One unique property of MSN is the ease and control at which the surfaces can be functionalized with organic moieties. The performance of the MSN in these sequestration applications is strongly dependent upon the surface functionalization; the interaction between the MSN and specific molecules will vary depending on the nature of the surface chemistry.

Herein, we report the synthesis of a series of mesoporous silica nanoparticles of similar pore diameters with various surface functional groups as sequestration nanomaterials for FFAs. The structure of the resulting materials were investigated via standard material characterization methods including powder $\mathrm{X}$-ray diffraction (XRD), transmission electron microscopy (TEM), scanning electron microscopy (SEM), and nitrogen sorption analysis. The sequestration capacity and FFA selectivity was demonstrated in a simulated feedstock oil with the optimized MSN material to determine the feasibility of using this material

Received: November 23, 2011

Accepted: January 11, 2012

Published: January 11, 2012 
for the isolation and purification of FFA from microalgal feedstocks. We discovered that aminopropyl functionalized MSN, with $10 \mathrm{~nm}$ pore diameter (AP-MSN-10), selectively sequestered FFAs. The high affinity of amines as adsorbents for organic acids ${ }^{21}$ coupled with the high surface area and uniform pore structure of the mesoporous material ${ }^{22}$ makes the aminefunctionalized mesoporous material an ideal candidate for FFA adsorption. Finally, we demonstrated the selective FFA sequestration ability of AP-MSN-10 from a crude, commercially produced algae oil feedstock.

\section{EXPERIMENTAL SECTION}

MSN-10. ${ }^{23}$ The nonionic surfactant Pluronic P104 (7.0 g) was added to $1.6 \mathrm{M} \mathrm{HCl}(273.0 \mathrm{~g})$. After stirring for $1 \mathrm{~h}$ at $55{ }^{\circ} \mathrm{C}$, tetramethylorthosilicate (TMOS, $10.64 \mathrm{~g}$ ) was added and stirred for an additional $24 \mathrm{~h}$. The resulting mixture was further posthydrothermally treated for $24 \mathrm{~h}$ at $150{ }^{\circ} \mathrm{C}$ in a high-pressure reactor. Upon cooling to room temperature, the white solid was collected by filtration, washed with copious amounts of methanol and dried in air. To remove the surfactant $\mathrm{P} 104$ by calcination, the MSN material was heated at a ramp rate of $1.5{ }^{\circ} \mathrm{C} \mathrm{min}{ }^{-1}$ and maintained at $550{ }^{\circ} \mathrm{C}$ for $6 \mathrm{~h}$.

$\mathbf{x}-\mathrm{MSN}-10$. Functionalized MSN-10 will be denoted as $\mathrm{x}-\mathrm{MSN}-10$, where $\mathrm{x}$ represents the organoalkoxysilane. Organoalkoxysilane $(3 \mathrm{mmol})$ was added to a toluene suspension $(100 \mathrm{~mL})$ of MSN-10 $(1.0 \mathrm{~g})$. The suspension was refluxed for $20 \mathrm{~h}$ under nitrogen. The resulting material was filtered, washed with toluene and methanol, and dried under vacuum overnight. The silanes used to functionalize the MSN10 were 3 -aminopropyltrimethoxysilane (AP), benzyltrimethoxysilane $(\mathrm{Bz})$, hexadecyltrimethoxysilane (Hex), 1-propyl-3-methyl imidazolium bromide trimethoxysilane (PMIm), and 3-mercaptopropyltrimethoxysilane (MP).

AP-Silica Gel. 3-aminopropyltrimethoxysilane $(3 \mathrm{mmol})$ was added to a toluene suspension $(100 \mathrm{~mL})$ of silica gel $(1.0 \mathrm{~g})$. The suspension was refluxed for $20 \mathrm{~h}$ under nitrogen. The resulting material was filtered, washed with toluene and methanol, and dried under a vacuum overnight.

Material Characterization. Powder X-ray diffraction experiments were performed on a Rigaku Ultima IV diffractometer using a $\mathrm{Cu} \mathrm{K} \alpha$ radiation source. Low angle diffraction with a $2 \theta$ range of $0.8-6^{\circ}$ was used to investigate the long-range order of the porous materials. TEM studies were done by placing a small aliquot of an aqueous suspension on a lacey carbon film coated 400 mesh copper grid and drying it in air. The TEM images were obtained on a Tecnai $\mathrm{F}^{2}$ microscope. Particle morphology was determined by SEM using a Hitachi S4700 FE-SEM system with $10 \mathrm{kV}$ accelerating voltage. The surface area and average pore diameter were measured using $\mathrm{N}_{2}$ adsorption/desorption measurements in a Micromeritics ASAP 2020 BET surface analyzer system. The data were evaluated using Brunauer-Emmett-Teller (BET) and Barrett-Joyner-Halenda (BJH) methods to calculate surface area and pore distributions, respectively. Samples were prepared by degassing at $100{ }^{\circ} \mathrm{C}$ overnight before analysis. Surface functional group loadings were determined by TGA. For TGA, $10 \mathrm{mg}$ samples were heated from 25 to $650{ }^{\circ} \mathrm{C}$ in air at a heating rate of $2{ }^{\circ} \mathrm{C} \mathrm{min}-1$ using a TA Instruments model 2950 thermogravimetric analyzer. The subsequent data were analyzed by Universal Analysis 2000 software. Zeta-potential measurements were carried out by sonicating $5 \mathrm{mg}$ of sample in $5 \mathrm{~mL}$ of PBS for $30 \mathrm{~min}$. The samples were then analyzed on a Malvern Instruments Zetasizer. All adsorption studies were done using GC-MS analysis. Samples were dissolved in hexanes and analyzed using an Agilent Technologies 7890A gas chromatograph equipped with a HP-5 ms column in-line with a 5975C mass detector.

Adsorption Experiments. Adsorption experiments were conducted using $16 \times 125 \mathrm{~mm}$ culture tubes. The adsorbent MSN material $(25 \mathrm{mg})$ was suspended in a $10 \mathrm{~mL}$ hexane solution containing either a single component or a mixture of components that make up the microalgal simulated solution for $6 \mathrm{~h}$. The adsorbate was separated from the solution using centrifugation and the supernatant was analyzed by GC-MS.

Microalgal Simulated Solution. A mixture of naturally occurring microalgal products ${ }^{7,8}$ was prepared to investigate the adsorption selectivity. Palmitic acid $(57 \mu \mathrm{M})$, glyceryl tristearate $(400 \mu \mathrm{M})$, squalene $(114 \mu \mathrm{M})$, and ergosterol $(57 \mu \mathrm{M})$ were all dissolved in a single solution of hexanes.

Microalgal Oil Adsorption. Crude microalgal oil obtained from Solix Biofuels, Inc., was used for testing the sequestration abilities of amine functionalized MSN-10 and amine functionalized silica gel. Silica material (100 or $300 \mathrm{mg}$ ) was added to $200 \mathrm{mg}$ of microalgal oil in $10 \mathrm{~mL}$ hexanes and mixed for $6 \mathrm{~h}$.

Methyl Esterification of Free Fatty Acids. Esterification of the MSN adsorbed FFAs was necessary for quantitative analysis via GC/ MS. The hexanes were removed under reduced pressure. To the resulting residue was added $0.01 \mathrm{M} \mathrm{HCl}$ in methanol $(4 \mathrm{~mL})$ and nonadecanoic acid, as an internal standard, and the mixture was stirred for $1 \mathrm{~h}$ at $80^{\circ} \mathrm{C}$. After the mixture was cooled to room temperature, $1 \% \mathrm{NaCl}(1 \mathrm{~mL})$ was added to the reaction mixture to increase the recovery of fatty acid methyl esters (FAMEs) from the reaction mixture by solvent extraction. The FAMEs were extracted with subsequent portions of hexane $(3 \times 3 \mathrm{~mL})$. The combined portions were diluted to a final volume of $10 \mathrm{~mL}$ with hexane and transferred to a GC vial for analysis by GC/MS.

Silylation of Sterols. ${ }^{24}$ Derivatization of sterols is necessary for quantification via GC/MS. The hexanes were removed under reduced pressure. Hexanes $(2 \mathrm{~mL})$ and $1 \mathrm{~mL}$ Sylon (BSTFA $(\mathrm{N}, \mathrm{O}-$ bis(trimethylsilyl)trifluoroacetamide) and TMCS (trimethylchlorosilane), 99:1) were added to the residue and heated to $70{ }^{\circ} \mathrm{C}$ for $1 \mathrm{~h}$ while stirring. The products were diluted to a final volume of $10 \mathrm{~mL}$ with hexane and transferred to GC vials for analysis by GC/MS.

Transesterification of Triacylglycerides. Transesterification of triacylglycerides are necessary for quantification via GC/MS. The hexanes were removed under reduced pressure and the resulting residue was dissolved in $4 \mathrm{~mL}$ of $0.04 \mathrm{M}$ methanolic $\mathrm{NaOH}$. The mixture was heated to $75{ }^{\circ} \mathrm{C}$ for $1 \mathrm{~h}$ while stirring. After the mixture was cooled to room temperature, $1 \% \mathrm{NaCl}(1 \mathrm{~mL})$ was added to the reaction mixture. The FAMEs were extracted with subsequent portions of hexane $(3 \times 3 \mathrm{~mL})$. The combined portions were diluted to a final volume of $10 \mathrm{~mL}$ with hexane and transferred to a $\mathrm{GC}$ vial for analysis by GC/MS.

Titration of Free Fatty Acids. The free fatty acids remaining in solution from the algal oil are quantified by titration with $\mathrm{KOH}$. The hexanes were removed under reduced pressure and resulting residue was dissolved in $125 \mathrm{~mL}$ isopropyl alcohol that was previously neutralized with $\mathrm{KOH}$. A few drops of phenolphthalein solution were added to the alcohol mixture and titrated with $0.05 \mathrm{M} \mathrm{KOH}$ until a color change was observed. The titration of FFAs was repeated to produce a triplicate of experiments.

\section{RESULTS AND DISCUSSION}

Screening of MSN Pore Size Dependence on FFA Sequestration. Initially, two mesoporous silica nanoparticle (MSN) materials with different pore sizes were synthesized and tested to determine the FFA sequestration dependence on pore diameter. Mesoporous silica nanoparticle material with a pore diameter of $2.5 \mathrm{~nm}$ (MSN-2.5) were synthesized following a previously reported method ${ }^{25}$ and MSN with $10 \mathrm{~nm}$ pores (MSN-10) were synthesized by modifying Linton's procedure. ${ }^{26}$ The two MSN materials were individually mixed with a hexane solution of FFAs for $6 \mathrm{~h}$ and the FFAs remaining in the supernatant were analyzed by GC-MS. The FFA sequestration capacity of MSN-2.5 and MSN-10 were 0.40 and $0.65 \mathrm{mmol}$ FFA $\mathrm{g}^{-1}$, respectively. Because MSN-10 was shown to sequester $63 \%$ more FFAs, it was chosen as the base material for continued studies.

Characterization of the $\mathrm{x}-\mathrm{MSN}-10 \mathrm{~s}$. The organoalkoxysilanes that were utilized to functionalize the MSN by postsynthetic 
Table 1. Structure Properties of MSN-10 and x-MSN-10

\begin{tabular}{|c|c|c|c|c|c|c|c|}
\hline sample & $d_{100}(\AA)$ & $a_{0}(\AA)$ & $W_{\mathrm{BJH}}{ }^{a}(\AA)$ & $S_{\mathrm{BET}}{ }^{a}\left(\mathrm{~m}^{2} \mathrm{~g}^{-1}\right)$ & $\mathrm{PV}^{a}\left(\mathrm{~cm}^{3} \mathrm{~g}^{-1}\right)$ & loading $^{b}\left(\mathrm{mmol} \mathrm{g}^{-1}\right)$ & zeta potential $(\mathrm{mV})$ \\
\hline MSN-10 & 100.38 & 115.91 & 104.7 & 375 & 1.02 & & -28.1 \\
\hline AP-MSN-10 & 92.02 & 106.25 & 100.7 & 290 & 0.78 & 1.54 & +19.3 \\
\hline Bz-MSN-10 & 90.09 & 104.03 & 101.0 & 266 & 0.72 & 1.25 & -19.8 \\
\hline Hex-MSN-10 & 86.63 & 100.03 & 98.3 & 265 & 0.94 & 0.68 & -21.7 \\
\hline PMIm-MSN-10 & 90.14 & 104.08 & 101.2 & 281 & 0.76 & 1.34 & +28.7 \\
\hline MP-MSN-10 & 91.85 & 106.06 & 100.3 & 233 & 0.86 & 1.20 & -22.4 \\
\hline
\end{tabular}

${ }^{a} W_{\mathrm{BJH}}$ represents the pore diameter; $S_{\mathrm{BET}}$ represents the surface area; PV represents the pore volume ${ }^{b}$ Loading represents the amount of functional group grafted on to the materials
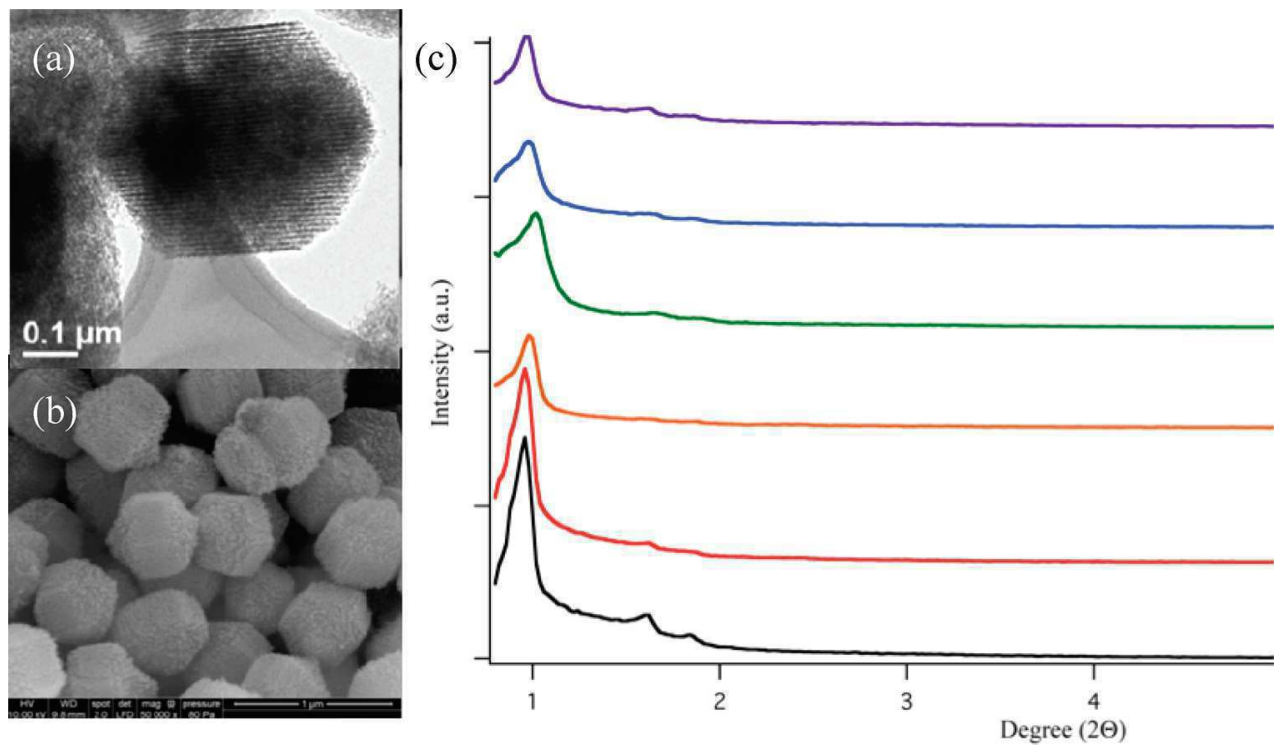

Figure 1. (a) Transmission electron micrograph, (b) scanning electron micrograph, and (c) XRD spectra of the surfactant removed unfunctionalized MSN-10 (black), AP-MSN-10 (red), Bz-MSN-10 (orange), Hex-MSN-10 (green), PMIm-MSN-10 (blue), and MP-MSN-10 (purple).

grafting were 3-aminopropyltrimethoxysilane (AP), benzyltrimethoxysilane $(\mathrm{Bz})$, hexadecyltrimethoxysilane (Hex), 1-propyl-3-methyl imidazolium bromide trimethoxysilane (PMIm), and 3-mercaptopropyltrimethoxysilane (MP). To functionalize the MSN-10, we added $3 \mathrm{mmol} \mathrm{g}^{-1}$ of each organoalkoxysilane to a refluxing toluene suspension of the mesoporous material. The amount of each functional group loaded on the surface of MSN-10 can be found in Table 1 as determined by TGA analysis. The X-ray diffraction patterns of $\mathrm{x}-\mathrm{MSN}-10$ are shown in Figure 1c. The observed diffraction patterns with intense $\left(d_{100}\right)$ peaks are characteristic of highly ordered two-dimensional (2D) hexagonal mesostructures with uniform channels. ${ }^{27}$ To directly visualize the pore structure, we analyzed the $\mathrm{x}$-MSN-10 materials by transmission electron microscopy (TEM). As shown in Figure 1a, the parallel stripes indicate that the postsynthetic grafting did not destroy the cylindrical mesoporous channels, ${ }^{17}$ which is consistent with the XRD measurements. The scanning electron micrograph (SEM) in Figure $1 \mathrm{~b}$ shows the particles have a spherical structure and not a flat, disk-like shape. ${ }^{26}$ The maintenance of the pore structure is also confirmed by nitrogen sorption analysis using $\mathrm{BET}$ and $\mathrm{BJH}$ calculations, see Figure 2 for isotherms. A small decrease in the pore diameter of appoximately $0.5 \mathrm{~nm}$ is observed after functionalization, along with a drop in surface area $\left(375 \mathrm{~m}^{2} \mathrm{~g}^{-1}\right.$ to $\left.290 \mathrm{~m}^{2} \mathrm{~g}^{-1}\right)$. Thermogravimetric analysis (TGA)
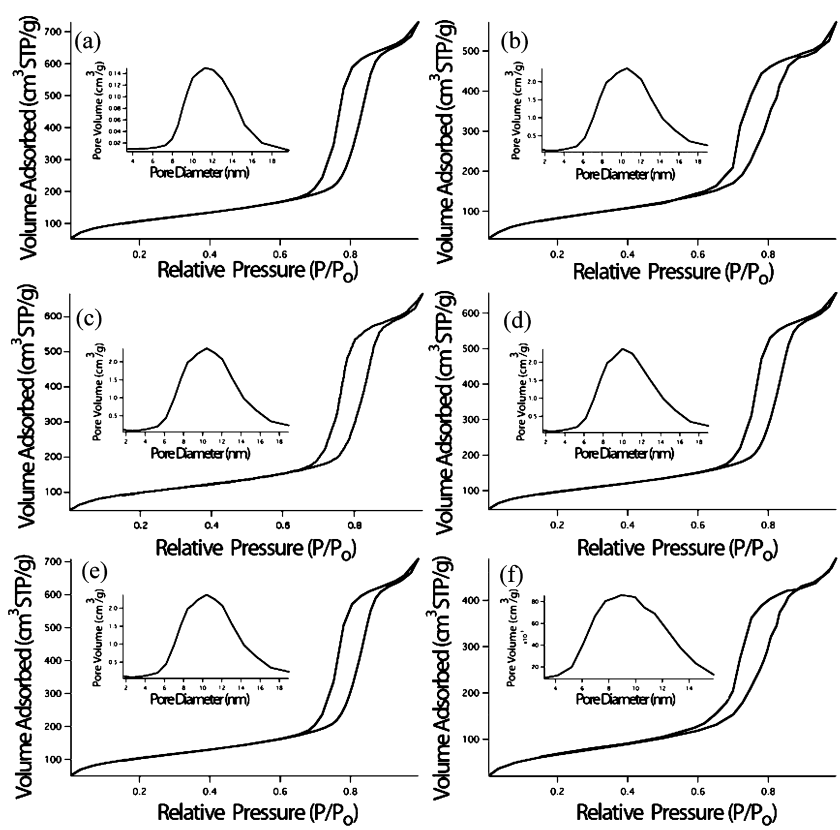

Figure 2. BET isotherms and $\mathrm{BJH}$ pore size distributions (insets) of MSN-10 (a), AP-MSN-10 (b), Bz-MSN-10 (c), Hex-MSN-10 (d), PMIm-MSN-10 (e), and MP-MSN-10 (f). 
of $\mathrm{x}-\mathrm{MSN}-10 \mathrm{~s}$ was utilized to determine the loading amounts of each organoalkoxysilane. A summary of all of the characterization measurements can be found in Table 1 .

Selection of the Optimal Functional Group for FFA Sequestration. To determine the organic functional group that optimized the effectiveness of our adsorbent material, the resulting $\mathrm{x}$-MSN-10 materials were mixed with a hexane solution of FFAs and the FFAs remaining in solution were analyzed. The results varied but AP-MSN-10 sequestered $98 \%$ of available FFA (0.98 mmol FFA g $\mathrm{MSN}^{-1}$ from a $1.0 \mathrm{mM}$ FFA solution), followed by Bz-MSN-10, which sequestered $0.50 \mathrm{mmol} \mathrm{FFA} \mathrm{g} \mathrm{MSN}^{-1}$, see Table 2 for a complete list of the

Table 2. Adsorption Dependence of FFAs on MSN Surface Grafted Functional Group

$\begin{array}{lcc}\begin{array}{c}\text { functional group } \\ \text { (x-trialkoxysilane) }\end{array} & \begin{array}{c}\text { amount adsorbed (mmol } \\ \left.\text { FFA g MSN }^{-1}\right)\end{array} & \begin{array}{c}\text { amount adsorbed } \\ \left(\mathrm{mmol} \mathrm{FFA} \mathrm{m}^{-2}\right)\end{array} \\ \text { none } & 0.65 & 0.0017 \\ \text { 3-aminopropyl } & 0.98 & 0.0034 \\ \text { benzyl } & 0.50 & 0.0019 \\ \text { hexadecyl } & 0.30 & 0.0011 \\ \text { 1-propyl-3-methyl } \\ \text { imidazolium bromide }\end{array}$

sequestration results by mass and surface area of MSN. The high sequestration capacity per unit of surface area of the APMSN-10 lends itself to be used as an adsorbent for FFAs.

Adsorption Isotherms. The kinetics of palmitic acid adsorption on AP-MSN-10 was measured (Figure 3) to

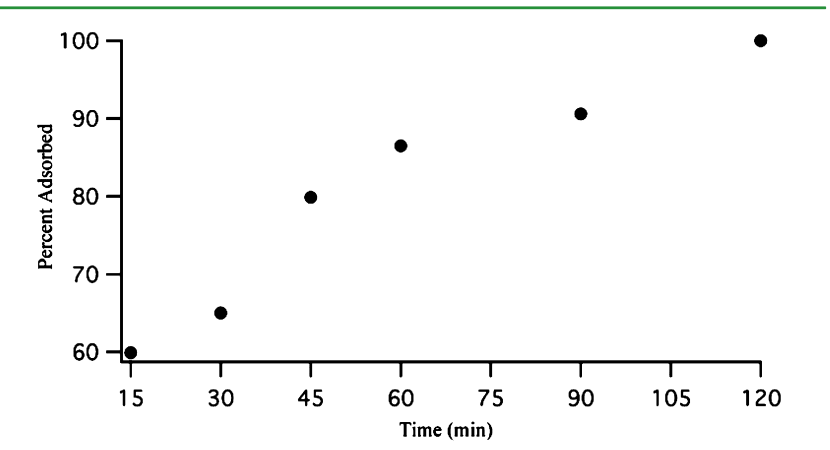

Figure 3. Amount of palmitic acid adsorbed on AP-MSN-10 as a function of contact time (initial [palmitic acid] $=2.1 \mathrm{mM}$ ).

determine the time required for equilibrium to be reached between the adsorbent and adsorbate. The amount of acid adsorbed on the AP-MSN-10 reached $100 \%$ at $120 \mathrm{~min}$. The adsorbent capacities of the sequestration materials were determined by treating unfunctionalized MSN-10 and AP-MSN-10 materials with different analytes in varying concentrations that are commonly found in microalgal solutions. The components of the simulated microalgal solution were palmitic acid (FFA), glyceryl tristearate (triacylglyceride), squalene (terpene), and ergosterol (sterol). Data found in Table 3 and the adsorption isotherms indicate AP-MSN-10 has a higher adsorption capacity for FFAs than unfunctionalized MSN-10. Interestingly, an adsorption capacity of $1.50 \mathrm{mmol} \mathrm{g}^{-1}$ is equal
Table 3. $Q_{\max }$ Values $\left(\mathrm{mmol}^{-1}\right)$ for Adsorbates on MSN-10 and AP-MSN-10

\begin{tabular}{lcc} 
& MSN-10 & AP-MSN-10 \\
palmitic acid & 0.81 & 1.50 \\
glyceryl tristearate & 0.44 & 0.22 \\
ergosterol & 0.48 & 0.18 \\
squalene & 0.0089 & 0.0063 \\
\hline
\end{tabular}

to the number of surface amine moieties on the surface of AP-MSN-10 (1.53 mmol $\left.\mathrm{g} \mathrm{MSN}^{-1}\right)$ as determined by TGA, indicating a 1:1 complexation between the surface amines and free fatty acids and monolayer coverage of the surface. All of the adsorption isotherms that were measured were fitted to the Langmuir equation

$$
Q=\frac{k_{1} C}{k_{2}+C}
$$

where $Q\left(\mathrm{mmol} \mathrm{g}^{-1}\right)$ is the amount of adsorbate adsorbed per gram of adsorbent, $C$ is the adsorbate concentration, and $k_{1}$ and $k_{2}$ are Langmuir adsorption equilibrium constants. The data was fitted to the equation with the coefficient of determination values exceeding 0.95 , indicating a statistical goodness of fit. The adsorption isotherms and Langmuir adsorption model curve fits are shown in Figures 4 and 5. In addition to improved FFA adsorption over unfunctionalized MSN-10, AP-MSN-10 had a significantly higher adsorption capacity for FFAs compared to the other classes of adsorbates. The nonfunctionalized MSN-10 reached an adsorption capacity of $0.81 \mathrm{mmol} \mathrm{g}^{-1}$ for palmitic acid compared to the maximum adsorption capacity of $1.50 \mathrm{mmol} \mathrm{g}^{-1}$ for palmitic acid on the AP-MSN-10. In addition, the adsorption capacity of $1.50 \mathrm{mmol} \mathrm{g}^{-1}$ is much higher than previous reports for porous silica materials ranging from 0.18 to $0.6 \mathrm{mmol} \mathrm{g}^{-1}$. $^{28-30}$

Selectivity of AP-MSN-10. To demonstrate the selectivity of AP-MSN-10 for FFAs, $25 \mathrm{mg}$ of AP-MSN-10 was mixed with $10 \mathrm{~mL}$ of a simulated solution closely resembling microalgal oil containing a mixture of palmitic acid $(57 \mu \mathrm{M})$, glyceryl tristearate $(400 \mu \mathrm{M})$, squalene $(114 \mu \mathrm{M})$, and ergosterol $(57 \mu \mathrm{M})$. After removal of the AP-MSN-10 from the solution, the hydrocarbons remaining in solution were quantified. The results of the selectivity experiments are listed in Table 4. AP-MSN-10 exhibited a dramatic preference for adsorbing palmitic acid over all the other substances, while MSN-10 adsorbed all substances equally except for squalene. This phenomenon can be explained by the surface amines of AP-MSN-10 acting as specific binding sites for FFAs. According to Bruckenstein and Untereker, an uncharged complex will form in hexane resulting from the exchange of the acidic proton from the FFA to the primary amine. $^{31}$ This phenomenon is used to explain the high selectivity of AP-MSN-10 toward FFAs. The other three classes of adsorbates do not contain an acidic proton or in the case of ergosterol, the $\mathrm{p} K_{\mathrm{a}}$ is too high to exchange with the surface amine. Although unfunctionalized MSN-10 did not demonstrate any selectivity, the incorporation of the amine group greatly shifted the selectivity of the mesoporous material toward free fatty acids.

Recyclability of AP-MSN-10. The recyclability of APMSN-10 was tested by repeated experiments of palmitic acid 


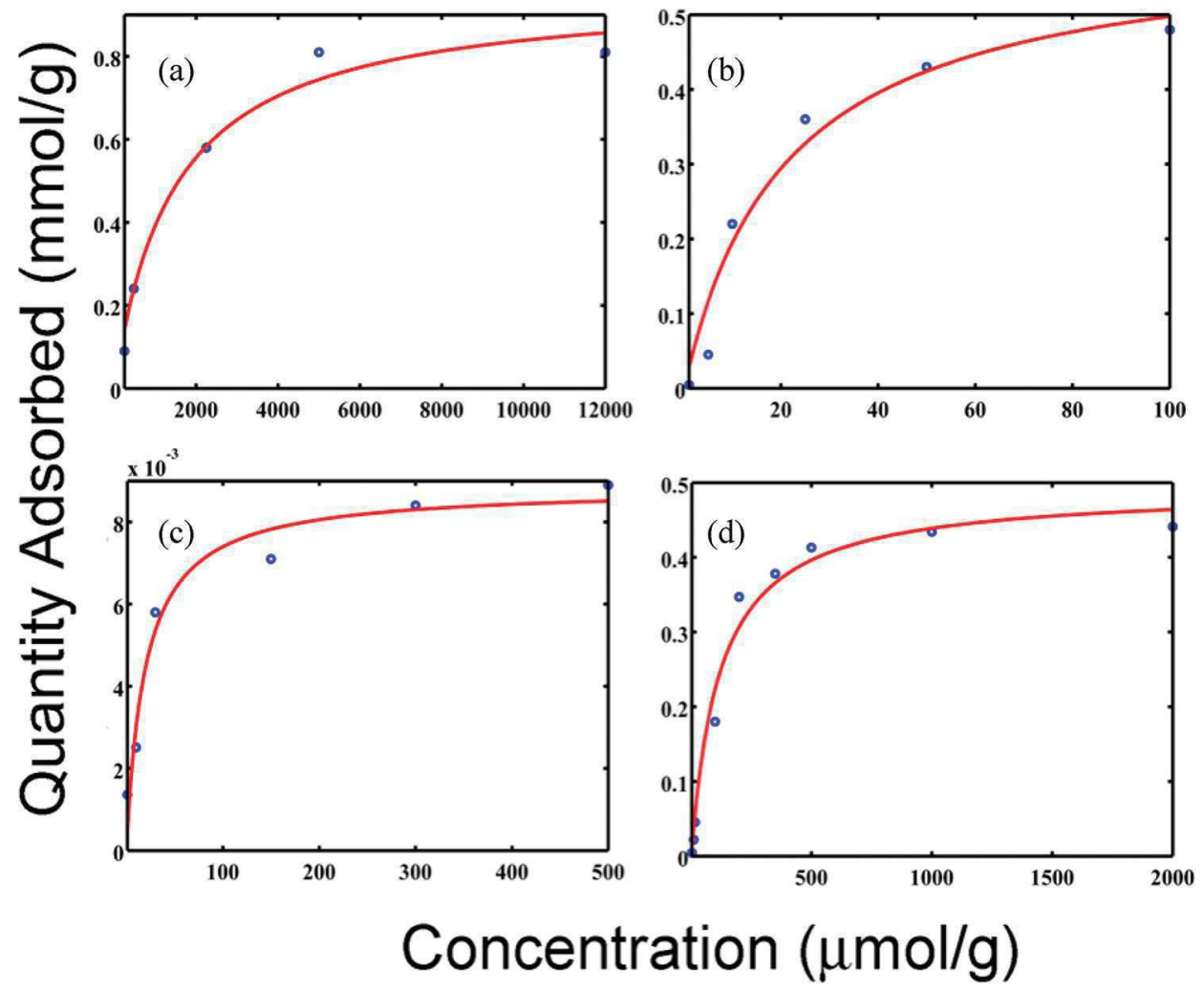

Figure 4. Adsorption isotherms of (a) palmitic acid, (b) ergosterol, (c) squalene, and (d) glyceryl tristearate adsorbed on MSN-10.

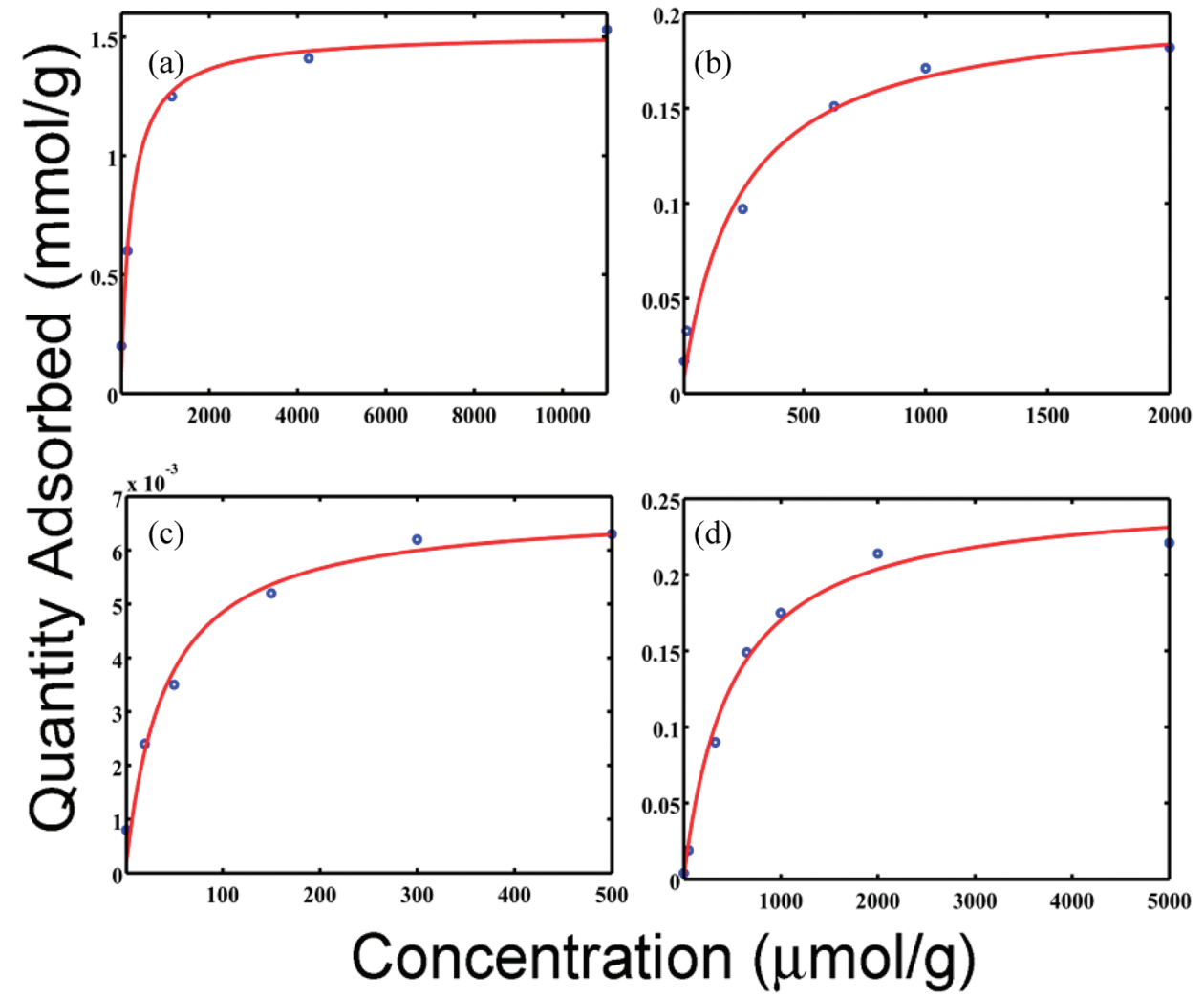

Figure 5. Adsorption isotherms of (a) palmitic acid, (b) ergosterol, (c) squalene, and (d) glyceryl tristearate adsorbed on AP-MSN-10.

adsorption. The AP-MSN-10 with adsorbed palmitic acid was added to the esterification reaction mixture to transform the acids to the methyl esters. By reacting the acidic proton involved in the complexation responsible for the acid selectivity, 
Table 4. Adsorption Values $\left(\mathrm{mmol} \mathrm{g}^{-1}\right)$ from Simulated Microalgal Oil for MSN-10 and AP-MSN-10

\begin{tabular}{lcccc} 
& MSN-10 & $\begin{array}{c}\text { MSN-10 } \\
\text { \% sequestered }\end{array}$ & AP-MSN-10 & $\begin{array}{c}\text { AP-MSN-10 } \\
\% \text { sequestered }\end{array}$ \\
$\begin{array}{l}\text { palmitic acid } \\
\text { glyceryl }\end{array}$ & 0.0228 & 100 & 0.0228 & 100 \\
tristearate & 0.150 & 93.8 & 0.012 & 7.5 \\
ergosterol & 0.0228 & 100 & 0.0039 & 17.1 \\
squalene & 0.0082 & 17.9 & 0.0048 & 10.5 \\
\hline
\end{tabular}

we can desorb the sequestered molecules from the surface of the AP-MSN-10. After the first cycle of adsorption, we are able to recover $92 \%$ of the adsorbed palmitic acid; however, the adsorption efficiency of the second and third cycles dropped to $73 \%$ and $59 \%$ of the first cycle, respectively. The decrease in adsorption efficiency is likely due to the remaining acid still adsorbed to the silica surface after desorption is attempted and the harsh conditions of the acid-catalyzed esterification deteriating the silica material. Active research is currently underway to identify less harsh, more efficient methods to recycle the sequestration MSN material.

Adsorption of FFAs by AP-MSN-10 from Commercial Microalgal Oil. To demonstrate the selective adsorption ability of the AP-MSN-10 with commercially available microalgal oil, $100 \mathrm{mg}$ AP-MSN-10 and $300 \mathrm{mg} \mathrm{AP}$-silica gel, as a control, were mixed with microalgal oil obtained from Solix, Inc. (Fort Collins, CO). Once the materials were separated from the oil, the supernatant was analyzed for remaining FFA content. Using varying amounts of material, AP-MSN-10 adsorbed $1.50 \mathrm{mmol}$ FFA $\mathrm{g}^{-1}$ compared to the $0.38 \mathrm{mmol} \mathrm{FFA} \mathrm{g}^{-1}$ for the AP-silica gel. The increase in surface area and pore volume by the incorporation of mesopores into the structure of the silica adsorbent plays a vital role in increasing the adsorption capacity. Both adsorption quantities closely match the amount of amine groups grafted on the surface of each material. The AP-MSN-10 is able to maintain the adsorption capabilities in a complex mixture applicable to current processes.

Further experiments were conducted using AP-MSN-10 for the adsorption of FFAs from commercial microalgal oil. By using the calculated value for amine moieties on the surface of AP-MSN-10, we determined the amount of material necessary to introduce a 1:1 ratio of amine groups to FFA in the microalgal oil. A $100 \mathrm{mg}$ aliquot of microalgal oil was dissolved in hexanes and mixed with $25 \mathrm{mg}$ of AP-MSN-10 for $2 \mathrm{~h}$. After separating the silica materials from the supernatant, the supernatant was added to a second vial for a second adsorption cycle. The remaining FFAs were analyzed by titration. The FFA content of the microalgal oil was reduced from $11.5 \%$ to $3.2 \%$ after the first cycle and $0.70 \%$ after the second cycle, calculated by $\mathrm{KOH}$ titration. Reducing the FFA content to less than $1 \%$ will increase the feasibility of using current industrial transesterification catalysts for microalgal biodiesel.

\section{CONCLUSIONS}

In summary, the successful synthesis and characterization of aminopropyl-functionalized $10 \mathrm{~nm}$ pore mesoporous silica nanoparticle material has been demonstrated. We showed that functionalizing $\mathrm{MSN}-10$ was necessary to optimize the sequestration capacity and selectivity for FFAs (see Scheme 1). The addition of the primary amine group to the surface of MSN10 increased the adsorption capacity from 0.8 to $1.50 \mathrm{mmol}$ FFA $\mathrm{g}^{-1}$ and improved the selectivity from 11 to $53 \mathrm{~mol} \%$ uptake by utilizing proton exchange to create specific binding sites for FFAs. We have also demonstrated the ability to reduce the FFA content of microalgal oil to less than $1 \%$ using AP-MSN-10, which makes it more favorable to be used as a biodiesel source. We are currently establishing a series of mesoporous nanomaterials that are specific for different biomolecules produced by microorganisms.

Scheme 1. Schematic Illustrating the Selective Uptake and Sequestration of the Free Fatty Acids from a Solution of Lipids and Hydrocarbons Found in Microalgal Oil

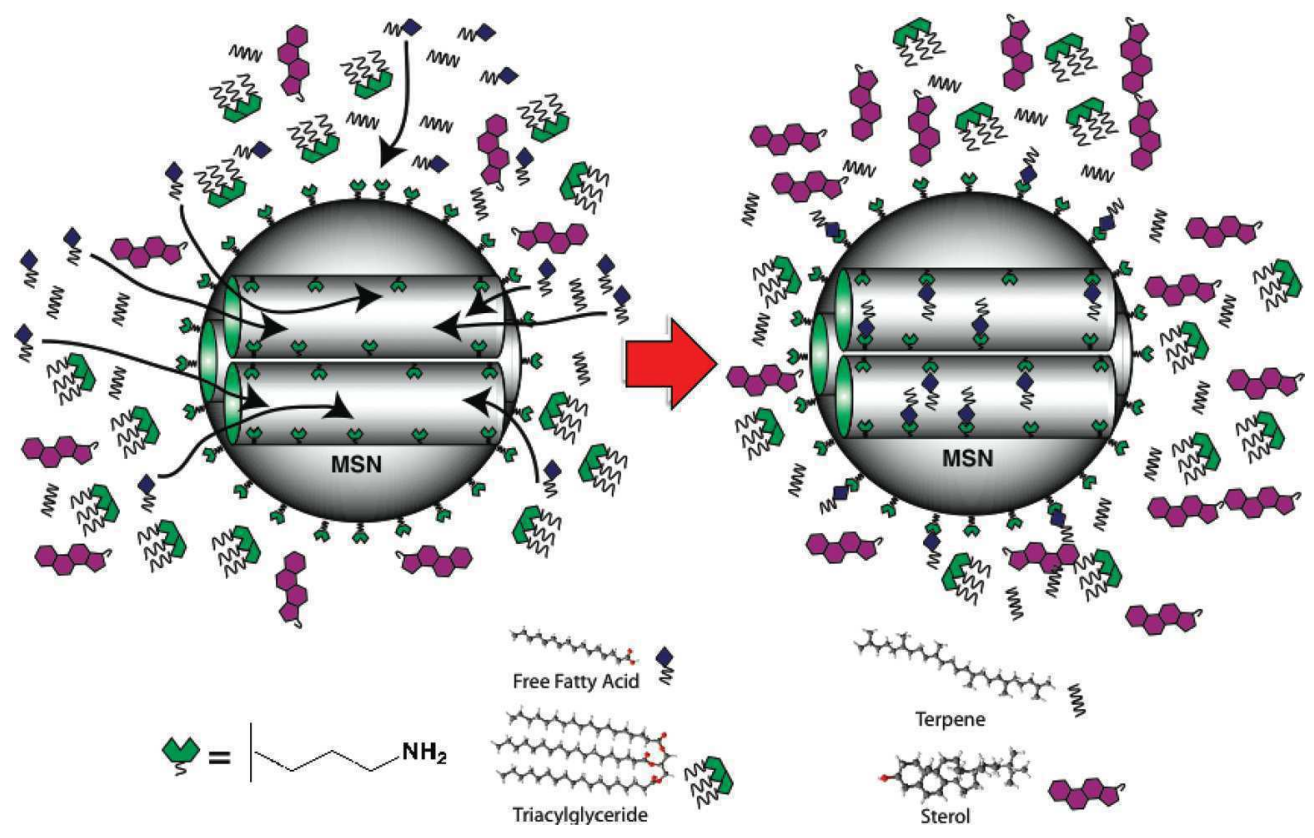




\section{AUTHOR INFORMATION}

\section{Corresponding Author}

*E-mail: bgtrewyn@iastate.edu.

\section{Notes}

${ }^{\dagger}$ Deceased May 4, 2010

\section{ACKNOWLEDGMENTS}

B.G.T., I.I.S., and J.S.V. thank the U.S. Department of Energy, Office of Energy Efficiency and Renewable Energy (Grant DE-FG26-0NT08854), for financial support. B.G.T., K.K., and F.M. also acknowledge funding of this work by the U.S. Department of Energy under Contract DE-EE0003046 awarded to the National Alliance for Advanced Biofuels and Bioproducts.

\section{REFERENCES}

(1) Gerpan, J. V. Fuel Process. Technol. 2005, 86 (10), 10.

(2) Yoshimura, T. Petrotech (Tokyo) 1998, 21 (10), 967-972.

(3) Zhang, Y.; Dube, M. A.; McLean, D. D.; Kates, M. Bioresour. Technol. 2003, 90 (3), 229-240.

(4) Cheng, Y.; Lu, Y.; Gao, C.; Wu, Q. Energy Fuels 2009, 23 (8), 4166-4173.

(5) Chisti, Y. Biotechnol. Adv. 2007, 25 (3), 294-306.

(6) Li, X.; Xu, H.; Wu, Q. Biotechnol. Bioeng. 2007, 98 (4), 764-771.

(7) Metzger, P.; Largeau, C. Appl. Microbiol. Biotechnol. 2005, 66 (5), 486-496.

(8) Zhila, N. O.; Kalacheva, G. S.; Volova, T. G. Russ. J. Plant Physiol. 2005, 52 (3), 311-319.

(9) Angenent, L. T.; Karim, K.; Al-Dahhan, M. H.; Wrenn, B. A.; Domiguez-Espinosa, R. Trends Biotechnol. 2004, 22 (9), 477-485.

(10) Bungay, H. R. Trends Biotechnol. 2004, 22 (2), 67-71.

(11) Wu, Z.; Yang, S.-T. Biotechnol. Bioeng. 2003, 82 (1), 93-102.

(12) Chen, H.-T.; Huh, S.; Wiench, J. W.; Pruski, M.; Lin, V. S. Y. J. Am. Chem. Soc. 2005, 127 (38), 13305-13311.

(13) Nieweg, J. A.; Lemma, K.; Trewyn, B. G.; Lin, V. S. Y.; Bakac, A. Inorg. Chem. 2005, 44 (16), 5641-5648.

(14) Zhao, Y.; Trewyn, B. G.; Slowing, I. I.; Lin, V. S. Y. J. Am. Chem. Soc. 2009, 131 (24), 8398-8400.

(15) Giri, S.; Trewyn, B. G.; Stellmaker, M. P.; Lin, V. S. Y. Angew. Chem., Int. Ed. 2005, 44 (32), 5038-5044.

(16) Lai, C.-Y.; Trewyn, B. G.; Jeftinija, D. M.; Jeftinija, K.; Xu, S.; Jeftinija, S.; Lin, V. S. Y. J. Am. Chem. Soc. 2003, 125 (15), 4451-4459.

(17) Radu, D. R.; Lai, C.-Y.; Jeftinija, K.; Rowe, E. W.; Jeftinija, S.; Lin, V. S. Y. J. Am. Chem. Soc. 2004, 126 (41), 13216-13217.

(18) Slowing, I. I.; Trewyn, B. G.; Lin, V. S. Y. J. Am. Chem. Soc. 2007, 129 (28), 8845-8849.

(19) Torney, F.; Trewyn Brian, G.; Lin Victor, S. Y.; Wang, K. Nat. Nanotechnol. 2007, 2 (5), 295-300.

(20) Trewyn, B. G.; Whitman, C. M.; Lin, V. S. Y. Nano Lett. 2004, 4

(11), 2139-2143.

(21) Jun, Y.-S.; Huh, Y. S.; Park, H. S.; Thomas, A.; Jeon, S. J.; Lee, E. Z.; Won, H. J.; Hong, W. H.; Lee, S. Y.; Hong, Y. K. J. Phys. Chem. C 2007, 111 (35), 13076-13086.

(22) Lin, V. S. Y.; Lai, C.-Y.; Huang, J.; Song, S.-A.; Xu, S. J. Am. Chem. Soc. 2001, 123 (46), 11510-11511.

(23) Kim, T.-W.; Slowing, I. I.; Chung, P.-W.; Lin, V. S.-Y. ACS Nano 2011, 5 (1), 360-366.

(24) Blau, K., Halket, J. Handbook of Derivatives for Chromatography, 2nd ed; John Wiley \& Sons: New York, 1993.

(25) Huh, S.; Wiench, J. W.; Yoo, J.-C.; Pruski, M.; Lin, V. S. Y. Chem. Mater. 2003, 15 (22), 4247-4256.

(26) Linton, P.; Alfredsson, V. Chem. Mater. 2008, 20 (9), 28782880.

(27) Zhao, D.; Huo, Q.; Feng, J.; Chmelka, B. F.; Stucky, G. D. J. Am. Chem. Soc. 1998, 120 (24), 6024-6036.

(28) Gao, Q.; Xu, W.; Xu, Y.; Wu, D.; Sun, Y.; Deng, F.; Shen, W. J. Phys. Chem. B 2008, 112 (7), 2261-2267.
(29) O’Connor, A. J.; Hokura, A.; Kisler, J. M.; Shimazu, S.; Stevens G. W.; Komatsu, Y. Sep. Purif. Technol. 2006, 48 (2), 197-201.

(30) Topallar, H.; Bayrak, Y. Turk. J. Chem. 1999, 23 (2), 193-198.

(31) Bruckenstein, S.; Untereker, D. F. J. Am. Chem. Soc. 1969, 91 (21), 5741-5. 


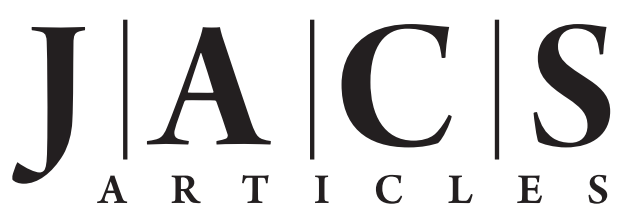

Published on Web 08/13/2010

\title{
Conformations of Silica-Bound (Pentafluorophenyl)propyl Groups Determined by Solid-State NMR Spectroscopy and Theoretical Calculations
}

\author{
Kanmi Mao, ${ }^{\dagger}$ Takeshi Kobayashi, ${ }^{\dagger}$ Jerzy W. Wiench, ${ }^{\dagger}$ Hung-Ting Chen, ${ }^{\dagger, \neq}$ \\ Chih-Hsiang Tsai, ${ }^{\ddagger}$ Victor S.-Y. Lin, ${ }^{\dagger, \ddagger}$ and Marek Pruski ${ }^{\star,+, \neq}$ \\ U.S. DOE Ames Laboratory, and Department of Chemistry, Iowa State University, \\ Ames, Iowa 50011
}

Received June 8, 2010; E-mail: mpruski@iastate.edu

\begin{abstract}
The conformations of (pentafluorophenyl)propyl groups $\left(-\mathrm{CH}_{2}-\mathrm{CH}_{2}-\mathrm{CH}_{2}-\mathrm{C}_{6} \mathrm{~F}_{5}\right.$, abbreviated as PFP), covalently bound to the surface of mesoporous silica nanoparticles (MSNs), were determined by solid-state NMR spectroscopy and further refined by theoretical modeling. Two types of PFP groups were described, including molecules in the prone position with the perfluorinated aromatic rings located above the siloxane bridges (PFP-p) and the PFP groups denoted as upright (PFP-u), whose aromatic rings do not interact with the silica surface. Two-dimensional (2D) ${ }^{13} \mathrm{C}-{ }^{1} \mathrm{H},{ }^{13} \mathrm{C}-{ }^{19} \mathrm{~F}$ and ${ }^{19} \mathrm{~F}-{ }^{29} \mathrm{Si}$ heteronuclear correlation (HETCOR) spectra were obtained with high sensitivity on natural abundance samples using fast magic angle spinning (MAS), indirect detection of low- $\gamma$ nuclei and signal enhancement by Carr-Purcell-Meiboom-Gill (CPMG) spin-echo sequence. 2D double-quantum (DQ) ${ }^{19} \mathrm{~F}$ MAS NMR spectra and spin-echo measurements provided additional information about the structure and mobility of the pentafluorophenyl rings. Optimization of the PFP geometry, as well as calculations of the interaction energies and ${ }^{19} \mathrm{~F}$ chemical shifts, proved very useful in refining the structural features of PFP-p and PFP-u functional groups on the silica surface. The prospects of using the PFP-functionalized surface to modify its properties (e.g., the interaction with solvents, especially water) and design new types of the heterogeneous catalytic system are discussed.
\end{abstract}

\section{Introduction}

In spite of a long history of organofluorine chemistry, fluorocarbons are of growing interest in academic studies and chemical industry. ${ }^{1-7}$ Due to high electronegativity and low electron polarizability of fluorine, the physical and chemical properties of these compounds differ from those of their hydrocarbon analogues. They exhibit extremely weak intermolecular van der Waals interactions, which gives rise to high volatility, low refractive index and small surface tension. ${ }^{8,9}$ Accordingly, modification of surface by fluorocarbons can influence the surface wettability. Indeed, a monolayer of $\mathrm{CF}_{3}\left(\mathrm{CF}_{2}\right)_{10} \mathrm{CO}_{2} \mathrm{H}$ molecules on platinum produced a nonwettable

${ }^{\dagger}$ Ames Laboratory, Iowa State University.

* Department of Chemistry, Iowa State University.

(1) Curran, D. P. Angew. Chem., Int. Ed. 1998, 37, 1175-1196

(2) Zhang, W.; Curran, D. P. Tetrahedron 2006, 62, 11837-11865.

(3) Dolbier, W. R., Jr. J. Fluorine Chem 2005, 126, 157-163.

(4) Lemal, D. M. J. Org. Chem. 2004, 69, 1-11.

(5) Uneyama, K.; Organofluorine Chemistry; Wiley-Blackwell: Oxford, U.K., 2006.

(6) Banks, R. E.; Smart, B. E.; Tatlow, J. C., Eds.; Organofluorine Chemistry: Principles and Commercial Applications; Springer: New York, 1994

(7) Hiyama, T. Organofluorine Compounds: Chemistry and Applications; Springer: New York, 2000.

(8) Persico, D. F.; Lagow, R. J.; Clark, L. C., Jr.; Huang, H.-N. J. Org Chem. 1985, 50, 5156-5159.

(9) Slinn, D. S. L.; Green, S. W. In Properties and Industrial Applications of Organofluorine Compounds; Banks, R. E., Ed.; Ellis Horwood: Chichester, 1982; pp 45-82. surface with the lowest tension value $(6 \mathrm{dyn} / \mathrm{cm})$ ever reported. ${ }^{10}$ Similarly, an "ultrahydrophobic" surface was formed on the silicon wafer via layer-by-layer sequential adsorption of perfluorinated polyelectrolytes. ${ }^{11}$ Several mesoporous silica materials with fluorocarbon-modified surfaces have been recently reported. ${ }^{12-14}$ For example, Corma and colleagues synthesized a mesoporous silica material with immobilized perfluorosulfonic acid and used it as a catalyst for esterification and acylation. The studies have shown that the surface properties of mesoporous channels can be strongly influenced by the presence of fluorocarbons, even when the surface coverage is only a fraction of a monolayer.

The goal of the present study is to demonstrate that the fundamental properties of surface-bound fluorocarbon molecules can be investigated in unprecedented detail by using the advanced two-dimensional solid-state NMR experiments and theoretical calculations. Specifically, we focus on understanding the molecular structure and conformation of the surface-bound PFP groups in the mesoporous silica nanoparticles (MSNs). The

(10) Hare, E. F.; Shafrin, E. G.; Zisman, W. A. J. Phys. Chem. 1954, 58, 236-239.

(11) Jisr, R. M.; Rmaile, H. H.; Schlenoff, J. B. Angew. Chem., Int. Ed. 2005, 44, 782-785.

(12) Kumar, R.; Chen, H.-T.; Escoto, J. L. V.; Lin, V. S. Y.; Pruski, M. Chem. Mater. 2006, 18, 4319-4327.

(13) Alvaro, M.; Corma, A.; Das, D.; Fornes, V.; Garcia, H. J. Catal. 2005, $231,48-55$

(14) Alvaro, M.; Corma, A.; Das, D.; Fornes, V.; Garcia, H. Chem. Commun. 2004, 956-957. 
NMR studies used newly developed schemes for enhancement of sensitivity under fast magic angle spinning (MAS), which enabled the detection of heteronuclear correlation (HETCOR) spectra of naturally abundant ${ }^{13} \mathrm{C}$ and ${ }^{29} \mathrm{Si}$ nuclei on the silica surface with exceptional sensitivity. The theoretical investigations provided invaluable assistance in refining the conformational details of two types of PFP species described by NMR.

\section{Materials and Methods}

PFP-MSN Material. The sample containing (pentafluorophenyl)propyl groups ( $-\mathrm{CH}_{2}-\mathrm{CH}_{2}-\mathrm{CH}_{2}-\mathrm{C}_{6} \mathrm{~F}_{5}$ ) covalently bound to MSNs, referred to as PFP-MSN, was synthesized via the previously reported cocondensation method. ${ }^{12,15-18}$ A mixture of cetyltrimethylammonium bromide ( $1.0 \mathrm{~g}, 2.75 \mathrm{mmol}), 2 \mathrm{M} \mathrm{NaOH}(\mathrm{aq})(3.5$ $\mathrm{mL}, 7.0 \mathrm{mmol}$ ), and $480 \mathrm{~mL}$ of $\mathrm{H}_{2} \mathrm{O}$ was stirred at $80{ }^{\circ} \mathrm{C}$ for 10 $\mathrm{min}$, followed by the addition of tetraethoxysilane (TEOS, $5 \mathrm{~mL}$, $22.4 \mathrm{mmol})$ and [(pentafluorophenyl)propyl]trimethoxysilane $(0.58$ $\mathrm{mL}, 2.24 \mathrm{mmol})$. The solution was maintained at $80{ }^{\circ} \mathrm{C}$ for $2 \mathrm{~h}$ and separated by filtration. The resulting product was sequentially washed with water, methanol, and dried under vacuum yielding $1.0 \mathrm{~g}$ of as-made PFP-MSN. The surfactant was removed using acid extraction, by refluxing the as-made PFP-MSN in methanolic solution of hydrochloric acid $(1.0 \mathrm{~mL}$ of concentrated $\mathrm{HCl}$ in 100 $\mathrm{mL}$ of $\mathrm{MeOH}$ ) for $6 \mathrm{~h}$. After filtration and extensive washing with water and methanol, $0.7 \mathrm{~g}$ of the surfactant-free PFP-MSN was obtained in the form of white fine powder. ${ }^{12}$

The TEM micrograph of PFP-MSN (not shown) indicated that the particles had elliptical shape with an average length of $300 \mathrm{~nm}$ and an aspect ratio of 1.5-2. The parallel, cylindrical pores formed a hexagonal mesostructure, as further confirmed by powder XRD experiments. The measured BET surface area and the pore diameter were $844 \pm 10 \mathrm{~m}^{2} / \mathrm{g}$ and $2.2 \pm 0.2 \mathrm{~nm}$, respectively. Based on the analysis of nitrogen adsorption, the mesopore volume was estimated at $0.75 \mathrm{~cm}^{3} / \mathrm{g}$.

Solid-State NMR. Solid-state NMR experiments were performed on a Varian NMR System spectrometer, equipped with a $1.6 \mathrm{~mm}$ triple resonance FastMAS probe and operated at $599.6 \mathrm{MHz}$ for ${ }^{1} \mathrm{H}, 564.2$ $\mathrm{MHz}$ for ${ }^{19} \mathrm{~F}, 150.8 \mathrm{MHz}$ for ${ }^{13} \mathrm{C}$ and $119.1 \mathrm{MHz}$ for ${ }^{29} \mathrm{Si}$ nuclei. The sample was packed in the MAS rotor after the exposure to ambient conditions in the laboratory. Several one-dimensional (1D) and twodimensional (2D) experiments were used, including 1D MAS with direct polarization (DPMAS), spin-echo, 2D through-bond and through-space HETCOR NMR utilizing the refocused INEPT (INEPTR) and cross-polarization (CP), respectively, and 2D doublequantum (DQ) MAS NMR. To increase the sensitivity, the ${ }^{1} \mathrm{H}-{ }^{13} \mathrm{C}$ and ${ }^{19} \mathrm{~F}-{ }^{13} \mathrm{C}$ HETCOR experiments were performed with indirect detection of heteronuclei $\left({ }^{13} \mathrm{C}\right)$ under fast MAS. ${ }^{19-21}$ The pulse sequences used in the indirectly detected through-space and throughbond HETCOR experiments are analogous to those reported elsewhere. ${ }^{20,21}$ The ${ }^{19} \mathrm{~F}-{ }^{29} \mathrm{Si}$ HETCOR spectra were acquired using cross-polarization and the Carr-Purcell-Meiboom-Gill (CPMG) refocusing of ${ }^{29} \mathrm{Si}$ magnetization. ${ }^{22,23}$

(15) Chen, H.-T.; Huh, S.; Lin, V. S. Y. In Catalyst Preparation; Regalbuto, J., Ed.; CRC Press: Boca Raton, 2007; pp 45-74.

(16) Chen, H. T.; Huh, S.; Wiench, J. W.; Pruski, M.; Lin, V. S. Y. J. Am Chem. Soc. 2005, 127, 13305-13311.

(17) Huh, S.; Chen, H. T.; Wiench, J. W.; Pruski, M.; Lin, V. S. Y. Angew. Chem., Int. Ed. 2005, 44, 1826-1830.

(18) Huh, S.; Chen, H. T.; Wiench, J. W.; Pruski, M.; Lin, V. S. Y. J. Am. Chem. Soc. 2004, 126, 1010-1011.

(19) Ishii, Y.; Tycko, R. J. Magn. Reson. 2000, 142, 199-204.

(20) Wiench, J. W.; Bronnimann, C. E.; Lin, V. S. Y.; Pruski, M. J. Am. Chem. Soc. 2007, 129, 12076-12077.

(21) Mao, K.; Wiench, J. W.; Lin, V. S. Y.; Pruski, M. J. Magn. Reson. 2009, 196, 92-95.

(22) Wiench, J. W.; Lin, V. S. Y.; Pruski, M. J. Magn. Reson. 2008, 193 , 233-242.

(23) Trebosc, J.; Wiench, J. W.; Huh, S.; Lin, V. S. Y.; Pruski, M. J. Am. Chem. Soc. 2005, 127, 7587-7593.

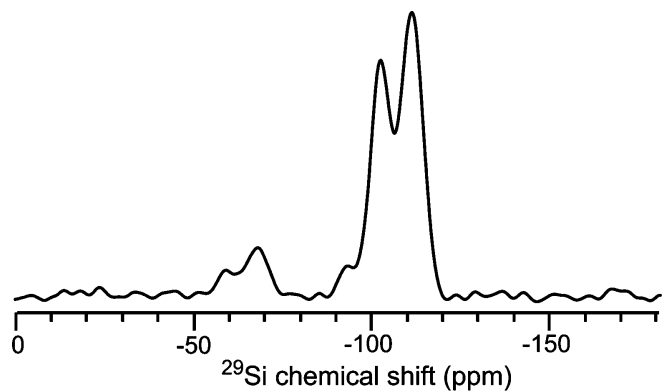

Figure 1. ${ }^{29} \mathrm{Si}$ DPMAS NMR spectrum of PFP-MSN taken under the following experimental conditions: $\nu_{\mathrm{R}}=10 \mathrm{kHz}, \nu_{\mathrm{RF}}^{\mathrm{Si}}=86 \mathrm{kHz}$ during short pulses, $\nu_{\mathrm{RF}}^{\mathrm{H}}=45 \mathrm{kHz}$ during TPPM decoupling, $N_{\mathrm{CPMG}}=25, \tau_{\mathrm{CPMG}}$ $=6 \mathrm{~ms}, \tau_{\mathrm{RD}}=300 \mathrm{~s}$ and $\mathrm{NS}=120$

The experimental parameters are given in figure captions, using the following symbols: $v_{\mathrm{R}}$ denotes the magic angle spinning (MAS) rate, $v_{\mathrm{RF}}^{\mathrm{X}}$ the magnitude of the RF magnetic field applied to $\mathrm{X}$ spins, $\tau_{\mathrm{CP}}$ the cross-polarization time, $\tau_{1}$ and $\tau_{2}$ the delays used during refocused INEPTR, $\tau_{\mathrm{RR}}$ the rotary resonance recoupling time, $N_{\mathrm{CPMG}}$ the number of echoes used during the CPMG acquisition, $\tau_{\mathrm{CPMG}}$ the time interval between $\pi$ pulses in the CPMG sequence, $\Delta t_{1}$ the increment of $t_{1}$ during 2D acquisition, NS the number of scans, $\tau_{\mathrm{RD}}$ the recycle delay, and AT the total acquisition time of a $2 \mathrm{D}$ spectrum. The ${ }^{1} \mathrm{H},{ }^{13} \mathrm{C}$ and ${ }^{29} \mathrm{Si}$ chemical shifts are reported using the $\delta$ scale and are referenced to TMS at $0 \mathrm{ppm}$. The ${ }^{19} \mathrm{~F}$ NMR spectra are referred to $\mathrm{CFCl}_{3}(\delta=0 \mathrm{ppm})$, based on substitution of the secondary reference of $\mathrm{NaF}$ aqueous solution $(-121.5$ ppm). ${ }^{24}$

Theoretical Calculations. The geometry of the PFP functional group inside the MSN was studied by theoretical calculations using $\mathrm{C}_{6} \mathrm{~F}_{6}$ and Si-containing cluster models for the surface oxygen sites of the MSN, as described in Results and Discussion. The geometries of the models were first approximated by the MM2 level calculations and further refined with the quantum mechanical theory using the Firefly program package. ${ }^{25,26}$ The quantum mechanical calculations were carried out at the Møller-Plesset second-order perturbation theory (MP2) level with the $6-311++\mathrm{G}(\mathrm{d}, \mathrm{p})$ basis set. The interaction energies in the optimized geometries were corrected for the basis set superposition error (BSSE) using the counterpoise method. ${ }^{27}$ Density functional theory (DFT) calculations of the NMR tensors were carried out at the B3LYP level using ORCA program package. ${ }^{28}$ An IGLO-II type basis set ${ }^{29}$ was employed in the DFT calculations. Theoretical shieldings were transformed to relative chemical shifts $\delta$ by subtracting the calculated chemical shift of $\mathrm{CFCl}_{3}$.

\section{Results and Discussion}

Solid-State NMR. The ${ }^{29}$ Si DPMAS spectrum of PFP-MSN (Figure 1) is dominated by resonance lines at around -113 and -104 ppm representing silicon sites $\mathrm{Q}^{4}\left((\equiv \mathrm{SiO}-)_{4} \mathrm{Si}\right)$ and $\mathrm{Q}^{3}$ $\left((\equiv \mathrm{SiO}-)_{3} \mathrm{Si}(-\mathrm{OH})\right)$, respectively. The presence of peaks centered at around -70 and $-60 \mathrm{ppm}$, which are assigned to silicon atoms in positions $(\mathrm{SiO})_{3} \mathrm{SiR}$ and $(\mathrm{SiO})_{2} \mathrm{Si}(\mathrm{OH}) \mathrm{R}$ (de-

(24) Chekmenev, E. Y.; Chow, S. K.; Tofan, D.; Weitekamp, D. P.; Ross, B. D.; Bhattacharya, P. J. Phys. Chem. B 2008, 112, 6285-6287.

(25) Granovsky, A. A. Firefly, version 7.1.G; http://classic.chem.msu.su/ gran/firefly/index.html.

(26) Schmidt, M. W.; Baldridge, K. K.; Boatz, J. A.; Elbert, S. T.; Gordon, M. S.; Jensen, J. H.; Koseki, S.; Matsunaga, N.; Nguyen, K. A.; Su, S. J.; Windus, T. L.; Dupuis, M.; Montgomery, J. A. J. Comput. Chem. 1993, 14, 1347-1363.

(27) Boys, S. F.; Bernardi, F. Mol. Phys. 1970, 19, 553-566.

(28) Nesse, F. ORCA-An Ab initio, DFT and Semiempirical Electronic Structure Package, Ver. 2.7.0; 2010.

(29) Kutzelnigg, W.; Fleischer, U.; Schindler, M. The IGLO-Method: Ab Initio Calculation and Interpretation of NMR Chemical Shifts and Magnetic Susceptibilities; Springer-Verlag: Heidelberg, 1990; Vol. 23. 

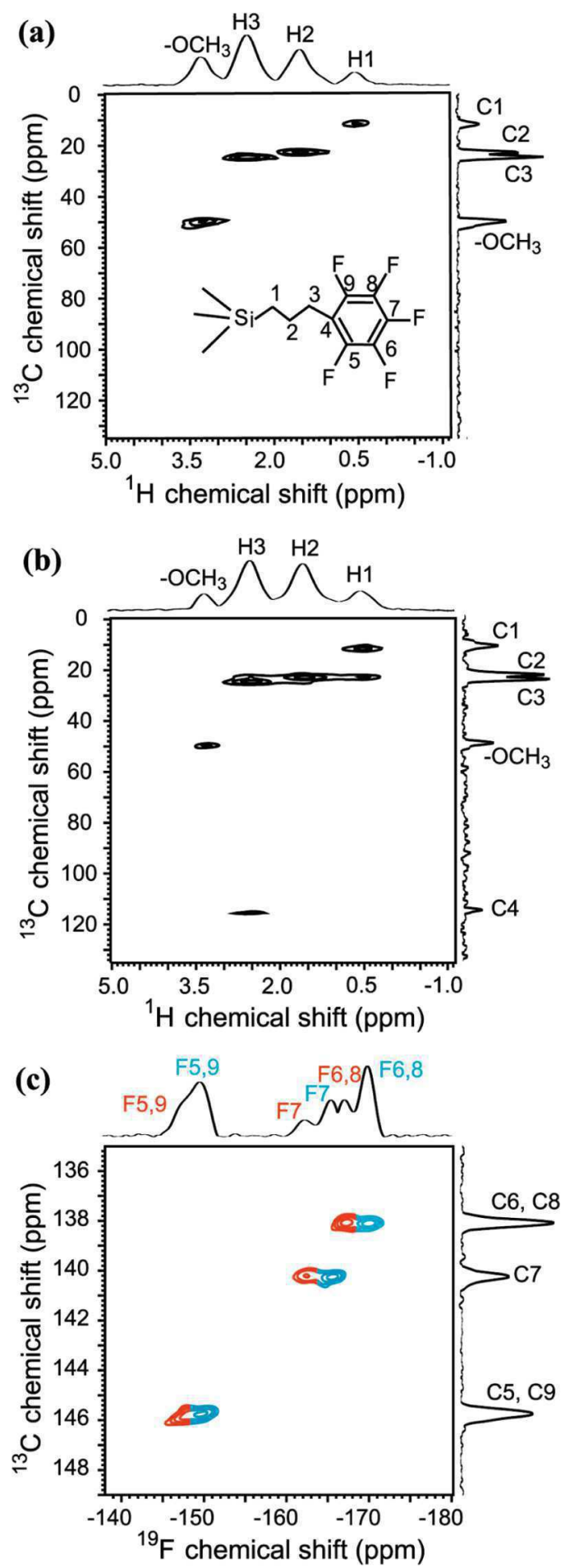

Figure 2. 2D indirectly detected ${ }^{13} \mathrm{C}-{ }^{1} \mathrm{H}(\mathrm{a}, \mathrm{b})$ and ${ }^{13} \mathrm{C}-{ }^{19} \mathrm{~F}(\mathrm{c})$ spectra of PFP-MSN. Spectra (a) and (b), obtained using through-bond (INEPTR) and through-space (CP) mixing, were used in our earlier report on indirect detection. ${ }^{21}$ They were recorded using $\nu_{\mathrm{R}}=40 \mathrm{kHz}, \nu_{\mathrm{RF}}^{\mathrm{H}}=110 \mathrm{kHz}$ during short pulses, $\nu_{\mathrm{RF}}^{\mathrm{H}}=60 \mathrm{kHz}$ during tangent ramp CP, $\nu_{\mathrm{RF}}^{\mathrm{H}}=20 \mathrm{kHz}$ during $\tau_{\mathrm{RR}}$, $\nu_{\mathrm{RF}}^{\mathrm{C}}=100 \mathrm{kHz}$ during short pulses and $\mathrm{CP}, \nu_{\mathrm{RF}}^{\mathrm{C}}=10 \mathrm{kHz}$ during SPINAL-64 decoupling, $\tau_{\mathrm{CP}}=4.5 \mathrm{~ms}, \tau_{1}=0.6 \mathrm{~ms}, \tau_{2}=0.8 \mathrm{~ms}, \tau_{\mathrm{RR}}=80 \mathrm{~ms}, 160$ rows with $\Delta t_{1}=25 \mu \mathrm{s}, 128$ (a) and 48 (b) scans per row, $\tau_{\mathrm{RD}}=1.5 \mathrm{~s}$, and $\mathrm{AT}=$ $12.5 \mathrm{~h} \mathrm{(a)}$ and $4.5 \mathrm{~h}$ (b). Spectrum (c) was obtained using INEPTR under similar conditions, exclusive of $\tau_{1}=1.0 \mathrm{~ms}, \tau_{2}=0.9 \mathrm{~ms}, 80$ rows, $\Delta t_{1}=200 \mu \mathrm{s}, 400$ scans per row, $\tau_{\mathrm{RD}}=0.8 \mathrm{~s}$, and $\mathrm{AT}=15.5 \mathrm{~h}$.

noted as $\mathrm{T}^{3}$ and $\mathrm{T}^{2}$ ), shows that the organic groups are indeed covalently bound to the surface. ${ }^{22}$ Based on integration of the ${ }^{29} \mathrm{Si}$ DPMAS spectrum we estimated that $13 \pm 2 \%$ of silicon

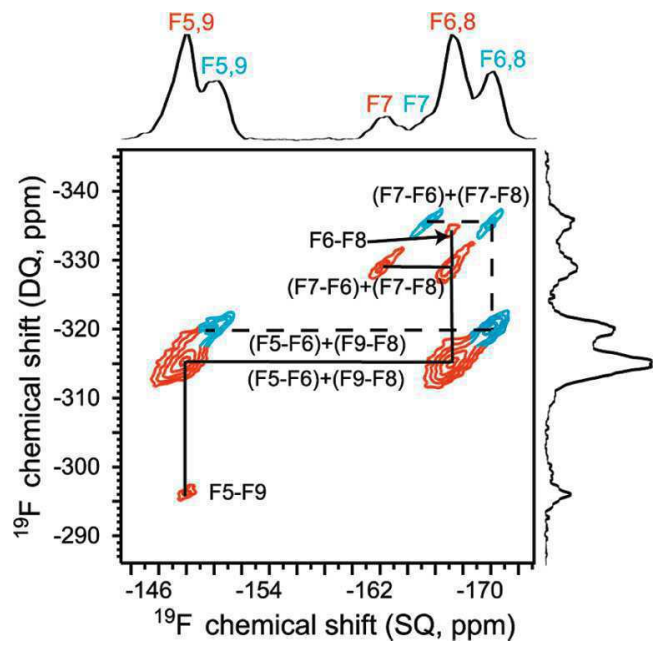

Figure 3. $2 \mathrm{D}{ }^{19} \mathrm{~F}-{ }^{19} \mathrm{~F}$ DQMAS spectrum of PFP-MSN. The DQ excitation and reconversion was achieved using back-to-back (BABA) pulse sequence, ${ }^{31} v_{\mathrm{R}}=40 \mathrm{kHz}, \nu_{\mathrm{RF}}^{\mathrm{F}}=110 \mathrm{kHz}$, and excitation/reconversion length $=0.4 \mathrm{~ms}$. The spectrum was acquired in 80 rows, with 128 scans per row, $\Delta t_{1}=25 \mu \mathrm{s}, \tau_{\mathrm{RD}}=1 \mathrm{~s}$, and $\mathrm{AT}=6 \mathrm{~h}$.

atoms in this sample are bound to carbon, which corresponds to the PFP concentration of $1.2 \mathrm{mmol} / \mathrm{g}$, or roughly 1 molecule per $\mathrm{nm}^{2}$. This last estimate was made by assuming that the inner pore surface area in PFP-MSN constitutes $90 \%$ of the total surface area of $844 \mathrm{~m}^{2} / \mathrm{g}$. Since the footprint area of the PFP complex is $\sim 0.6 \mathrm{~nm}^{2}$, this concentration would correspond to surface coverage of around $60 \%$, if all molecules assumed the prone position.

With the static ${ }^{1} \mathrm{H}$ and ${ }^{19} \mathrm{~F}$ linewidths being on the order of $10-20 \mathrm{kHz}$, the 2D HETCOR spectra of PFP-MSN could be best measured using the indirect detection of heteronuclei under fast MAS. ${ }^{19-21}$ The indirectly detected, INEPTR-based ${ }^{13} \mathrm{C}-{ }^{1} \mathrm{H}$ spectrum of PFP-MSN is shown in Figure 2a. The observed correlations between directly bound $\mathrm{C} 1-\mathrm{H} 1, \mathrm{C} 2-\mathrm{H} 2$ and C3-H3 pairs are consistent with the expected structure of the PFP groups covalently attached to the silica surface. Also observed is a resonance due to surface methoxy functionality, as shown in our earlier report. ${ }^{21}$ The same correlations, as well as those due to long-range, through-space interactions between $\mathrm{C} 2-\mathrm{H} 1, \mathrm{C} 2-\mathrm{H} 3$ and $\mathrm{C} 3-\mathrm{H} 2$ are observed in the spectrum derived thorough space with $\tau_{\mathrm{CP}}=4.5 \mathrm{~ms}$ (Figure $2 \mathrm{~b}$ ). Also observed in Figure $2 b$ is the correlation between $\mathrm{H} 3$ and the aromatic ring (carbon $\mathrm{C} 4$ ).

In the ${ }^{13} \mathrm{C}-{ }^{19} \mathrm{~F}$ HETCOR spectrum shown in Figure 2c, the aromatic carbons in PFP-MSN are observed through $J$ couplings with the directly bound fluorines. The observed ${ }^{13} \mathrm{C}$ shifts are consistent with those expected for carbons C5-C9. Surprisingly, each ${ }^{13} \mathrm{C}$ resonance is involved in double cross-peaks along the ${ }^{19} \mathrm{~F}$ dimension, labeled in red and blue in Figure 2c, representing fluorine sites with similar but discernible chemical shifts. This suggests the presence of two PFP functionalities, which reside on the silica surface in separate average environments and do not change on the time scale of $(\Delta v)^{-1} \cong 1 \mathrm{~ms}$ (where $\Delta v$ is the difference between the corresponding ${ }^{19} \mathrm{~F}$ resonance frequencies in both species). For reasons to be explained later, we denote the molecules represented by "blue" and "red" resonances as PFP-u and PFP-p.

The presence of two nonexchanging conformations of PFP is further verified by the $2 \mathrm{D}{ }^{19} \mathrm{~F}-{ }^{19} \mathrm{~F}$ DQMAS measurement (Figure 3), which under the excitation and refocusing conditions used here (see figure caption) found only intramolecular 


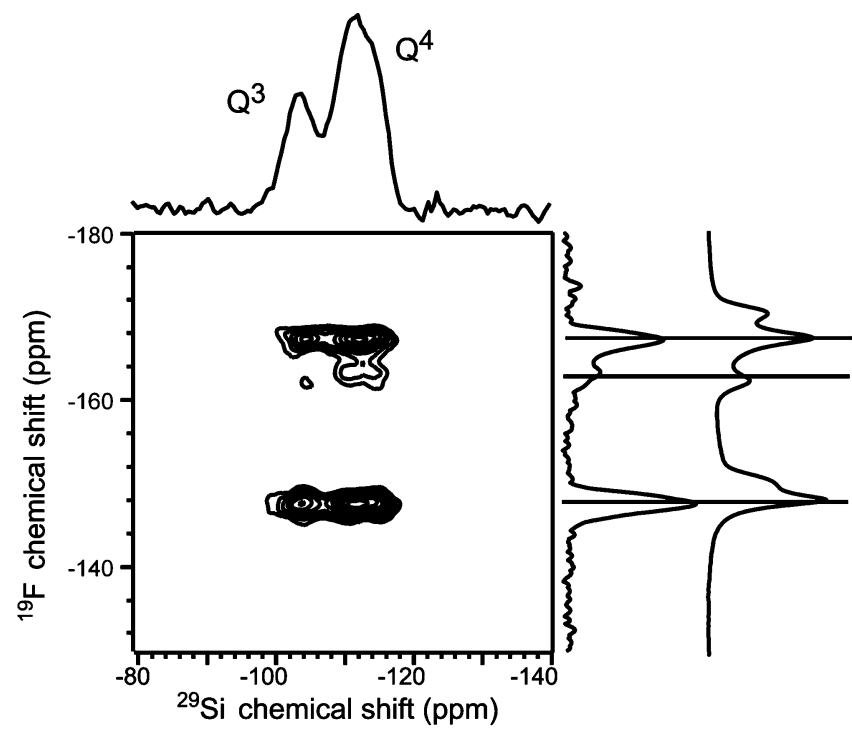

Figure 4. $2 \mathrm{D}{ }^{19} \mathrm{~F}-{ }^{29} \mathrm{Si}$ CP-based CPMG-HETCOR spectrum of PFP-MSN obtained using $v_{\mathrm{R}}=40 \mathrm{kHz}, \nu_{\mathrm{RF}}^{\mathrm{F}}=110 \mathrm{kHz}$ during short pulses, $\nu_{\mathrm{RF}}^{\mathrm{F}}=60$ $\mathrm{kHz}$ during tangent $\mathrm{CP}, \nu_{\mathrm{RF}}^{\mathrm{Si}}=100 \mathrm{kHz}$ during short pulses and $\mathrm{CP}, \tau_{\mathrm{CP}}=$ $20 \mathrm{~ms}, N_{\mathrm{CPMG}}=250$ and $\tau_{\mathrm{CPMG}}=4 \mathrm{~ms}$. The data were acquired in 40 rows, with $\Delta t_{1}=25 \mu \mathrm{s}, 2000$ scans per row, $\tau_{\mathrm{RD}}=0.8 \mathrm{~s}$, and AT $=47 \mathrm{~h}$ Also shown is the corresponding $1 \mathrm{D}{ }^{19} \mathrm{~F}$ MAS spectrum.

correlations between ${ }^{19} \mathrm{~F}$ nuclei, via the dipole-dipole coupling. In the DQ dimension, the signals representing two coupled nuclei resonating at $\omega_{1}$ and $\omega_{2}$ occur at the sum frequency $\omega_{1}$ $+\omega_{2}$, whereas the signals from non-interacting nuclei are suppressed by the DQ filtering. ${ }^{30}$ Thus, the spatial proximities between ${ }^{19} \mathrm{~F}$ nuclei can be identified by following the crosspeaks along the horizontal and vertical pathways, as shown in Figure 3 for PFP-p (solid lines) and PFP-u (dashed lines). In the PFP-p functional groups, correlations between all intramolecular neighbors are observed, including the diagonal crosspeaks $\mathrm{F} 5-\mathrm{F} 9$ and $\mathrm{F} 6-\mathrm{F} 8$, and the off-diagonal superpositions of $($ F5 -F6 $)+($ F8 $-F 9)$ and $($ F7 $-F 6)+($ F7-F8). Similar spectral features are observed for PFP-u, except for the diagonal peaks F5-F9 and F6-F8, which barely exceed the noise level. The differences between relative intensities in ${ }^{19} \mathrm{~F}$ projections in Figures $2 c$ and 3 are insignificant because they result from different spin dynamics involved in ${ }^{13} \mathrm{C}-{ }^{19} \mathrm{~F}$ HETCOR and ${ }^{19} \mathrm{~F}-{ }^{19} \mathrm{~F}$ DQ MAS experiments.

To delineate the nature of PFP-p and PFP-u species, we probed their interaction with the silica surface, by measuring a 2D ${ }^{19} \mathrm{~F}-{ }^{29} \mathrm{Si}$ HETCOR spectrum of PFP-MSN (Figure 4). The measurement was enabled by using the CPMG refocusing of ${ }^{29} \mathrm{Si}$ magnetization, ${ }^{22}$ which afforded a 6 -fold sensitivity gain (note that without the CPMG refocusing this spectrum would require two months of acquisition time). Notwithstanding the fact that both PFP-p and PFP-u were present in the sample, as evidenced by the ${ }^{19} \mathrm{~F}$ MAS spectrum shown along the $\omega_{1}$ dimension, the only observed ${ }^{19} \mathrm{~F}-{ }^{29} \mathrm{Si}$ correlations are associated with the PFP-p species. This demonstrates that the difference between PFP-p and PFP-u functional groups is in their interaction with the surface to which both are covalently bound. All ${ }^{19} \mathrm{~F}$ nuclei in PFP-p groups correlate with $\mathrm{Q}^{3}$ and $\mathrm{Q}^{4}$ silicon sites, which indicates that they reside in prone positions with respect to the silica surface and are less mobile. The lack

(30) Brown, S. P.; Spiess, H. W. Chem. Rev. 2001, 101, 4125-4155.

(31) Feike, M.; Demco, D. E.; Graf, R.; Gottwald, J.; Hafner, S.; Spiess, H. W. J. Magn. Reson. A 1996, 122, 214-221.

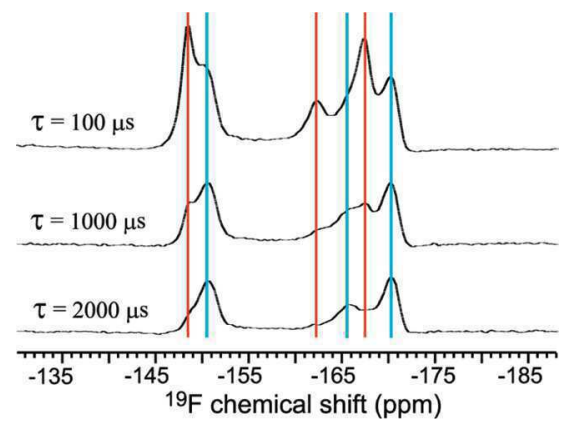

Figure 5. ${ }^{19} \mathrm{~F}$ spectra of dried PFP-MSN obtained under MAS at $40 \mathrm{kHz}$ using the spin-echo sequence with $\tau=0.1,1.0$, and $2.0 \mathrm{~ms}$.

of ${ }^{19} \mathrm{~F}-{ }^{29} \mathrm{Si}$ polarization transfer from the $\mathrm{PFP}-\mathrm{u}$ species suggests that their aromatic rings are not located near the surface. The increased mobility of the PFP-u species may further contribute to weakening of the ${ }^{19} \mathrm{~F}-{ }^{29} \mathrm{Si}$ dipole-dipole interactions (see the discussion of relaxation data and static NMR spectrum, below). No correlations involving the $\mathrm{T}$ sites and the aromatic ring were detected.

The ${ }^{13} \mathrm{C}-{ }^{19} \mathrm{~F}$ HETCOR spectrum of Figure $2 \mathrm{c}$ shows that the interaction with the surface shifts the ${ }^{19} \mathrm{~F}$ resonances in PFP-p by 2 to $3 \mathrm{ppm}$ in the direction of higher frequency (deshielded) with respect to PFP-u molecules, but has a very small effect $(<0.1 \mathrm{ppm})$ on the ${ }^{13} \mathrm{C}$ chemical shifts. An earlier study showed that adsorption of fluoroaromatic molecules (hexafluorobenzene, $\mathrm{C}_{6} \mathrm{~F}_{6}$ ) on the surface of alumina has a deshielding effect on the ${ }^{19} \mathrm{~F}$ nuclei compared to the same molecules in the gas phase. ${ }^{32}$

Further corroboration of these assignments was obtained by measuring the transverse relaxation time $\left(T_{2}{ }^{\prime}\right)$ of ${ }^{19} \mathrm{~F}$ nuclei under the spin-echo sequence $\pi / 2-\tau-\pi-\tau$-detect. The $T_{2}^{\prime}$ values obtained under fast MAS for PFP-u exceeded those for PFP-p by a factor of $\sim 8(12 \mathrm{~ms}$ versus $1.5 \mathrm{~ms})$. Indeed, the spectra in Figure 5 show that nuclei in PFP-u and PFP-p were refocused with $\tau=0.1 \mathrm{~ms}$, but only those in PFP-u were observed with $\tau=2 \mathrm{~ms}$, which is consistent with the increased mobility. The existence of two populations of PFP species with different mobilities can be also inferred from the analysis of static ${ }^{19} \mathrm{~F}$ NMR spectrum (not shown) consisting of two superimposed components with the linewidths of $16 \pm 2 \mathrm{kHz}$ and $6 \pm 1 \mathrm{kHz}$, which are mainly due to homonuclear ${ }^{19} \mathrm{~F}-{ }^{19} \mathrm{~F}$ dipolar interactions. This is a crude approximation, as each component is a superposition of 3 resonances representing F5,9, F6,8 and F7. Still, these values are close, respectively, to those expected for perfluotinated rings that are rigid (PFP-p) and undergo limited motion, such as librational rotation (PFP-u). Note, however, that such mobility alone should not prevent the PFP-u species from being observed in the $2 \mathrm{D}{ }^{19} \mathrm{~F}-{ }^{29} \mathrm{Si}$ HETCOR spectrum.

In short, solid-state NMR spectroscopy provided ample evidence for the existence of two distinct populations of PFP functional groups in the MSNs, both of which are covalently bound to the surface, have the same bond structure, but reside in positions described as "prone" (PFP-p) and "upright" (PFPu) inside the mesopores. The NMR data provide several additional insights about the physicochemical nature of these species. The existence of separate sets of ${ }^{19} \mathrm{~F}$ peaks representing both species shows that they do not change their conformations on the NMR time scale of $\sim 1 \mathrm{~ms}$. Based on the ${ }^{19} \mathrm{~F}-{ }^{29} \mathrm{Si} \mathrm{CP}$ efficiency, we can estimate that the PFP-p groups are located

(32) Budarin, V. L.; Clark, J. H.; Hale, S. E.; Tavener, S. J.; Mueller, K. T.; Washton, N. M. Langmuir 2007, 23, 5412-5418. 
within $\sim 0.45 \mathrm{~nm}$ from the nearest $\mathrm{Q}^{3}$ and $\mathrm{Q}^{4}$ silicon sites. This is a rough approximation, based on the maximum CP intensity being observed at $\tau_{\mathrm{CP}}=20 \mathrm{~ms}$ and the assumption that the $\mathrm{CP}$ dynamics for an rigid pair of spins reaches maximum at $\tau_{\mathrm{CP} \text {,opt }}$ $=1.7 / D$, where the dipolar coupling $D$ depends on the internuclear distance. $^{33}$

Theoretical Modeling. To propose a model of the geometry of the PFP-p species inside the MSNs, we support the solidstate NMR results with theoretical calculations. We point out that the surface of mesoporous silica comprises a complex network of Q and T sites with widely distributed ranges of ${ }^{29} \mathrm{Si}$ chemical shifts, which reflect their diverse local environments. Our aim is to put forth a simplified model that incorporates the basic chemical properties of the PFP-MSN surface and is consistent with both experimental and theoretical studies. Earlier $a b$ initio studies of the interaction between an isolated pair of water and $\mathrm{C}_{6} \mathrm{~F}_{6}$ molecules have shown that $\mathrm{C}_{6} \mathrm{~F}_{6}$ can act as a lone-pair acceptor and interact with the oxygen center of $\mathrm{H}_{2} \mathrm{O}$ forming a dimer with a binding energy of about $-8 \mathrm{~kJ} / \mathrm{mol}$. $^{34}$ Due to the electron-withdrawing effect of the fluorine atoms and the resulting electron deficiency of the $\mathrm{C}_{6} \mathrm{~F}_{6}$ aromatic $\pi$-system, the oxygen of water is located approximately 0.32 $\mathrm{nm}$ from the center of the molecule, with both hydrogen atoms pointing away from the ring. This is in contrast to water-benzene interaction, which leads to a different $\pi$-electron distribution and results in formation of a weak hydrogen bond with water, such that both hydrogen atoms point toward the ring. ${ }^{34}$ Following the Lewis acid and base concept, in the $\mathrm{C}_{6} \mathrm{~F}_{6}-\mathrm{H}_{2} \mathrm{O}$ system the oxygen atom acts as a Lewis base and the aromatic ring may exhibit Lewis acidity, whereas in the $\mathrm{C}_{6} \mathrm{H}_{6}-\mathrm{H}_{2} \mathrm{O}$ system water plays the role of an acid. These properties of the PFP functional groups are relevant to surface science and catalysis, as will be pointed out in the Conclusion.

The above-mentioned ab initio studies of the $\mathrm{C}_{6} \mathrm{~F}_{6}-\mathrm{H}_{2} \mathrm{O}$ system $^{34}$ evoke a possibility that pentafluorophenyl groups of the PFP functionalities act in a similar fashion as a lone-pair acceptor and interact with the oxygen atom of the MSN surface. We have optimized the geometries of $\mathrm{C}_{6} \mathrm{~F}_{6}$ interacting with two major oxygen species on the MSN surface: the oxygen atoms of silanol groups, $\mathrm{HO}-\mathrm{Si}(-\mathrm{OSi} \equiv)_{3}$, and siloxane bridges, $(\equiv \mathrm{SiO}-)_{3} \mathrm{Si}-\mathrm{O}-\mathrm{Si}(-\mathrm{OSi} \equiv)_{3}$. The surface models for these species were created based on $\beta$-cristobalite structure $(\mathrm{Si}-\mathrm{O}$ bond length $=0.161 \mathrm{~nm}$ and $\mathrm{Si}-\mathrm{O}-\mathrm{Si}$ bond angle $\left.=146.4^{\circ}\right),{ }^{35,36}$ because the local structure of MSN is similar to that of amorphous silica ${ }^{37,38}$ and X-ray work on amorphous silicas suggests that their structure most closely resembles that of $\beta$-cristobalite. ${ }^{39}$ The $\mathrm{Si}-\mathrm{O}-\mathrm{H}$ bond angle was initially set to $121^{\circ} .{ }^{40}$ The silicon atoms that do not carry the full complement of hydroxyl or siloxane bridges were terminated by hydrogen

(33) Amoureux, J. P.; Pruski, M. Mol. Phys. 2002, 100, 1595-1613.

(34) Danten, Y.; Tassaing, T.; Besnard, M. J. Phys. Chem. A 1999, 103, 3530-3534.

(35) Shimura, T.; Misaki, H.; Umeno, M.; Takahashi, I.; Harada, J. J. Cryst. Growth 1996, 166, 786-791.

(36) Peacor, D. R. Z. Kristallogr. 1973, 138, 274-298.

(37) Beck, J. S.; Vartuli, J. C.; Roth, W. J.; Leonowicz, M. E.; Kresge, C. T.; Schmitt, K. D.; Chu, C. T. W.; Olson, D. H.; Sheppard, E. W.; McCullen, S. B.; Higgins, J. B.; Schlenker, J. L. J. Am. Chem. Soc. 1992, 114, 10834-10843.

(38) Edler, K. J.; Reynolds, P. A.; White, J. W. J. Phys. Chem. B 1998, 102, 3676-3683.

(39) Frondel, C. Am. Mineral. 1979, 64, 799-804.

(40) Kobayashi, T.; DiVerdi, J. A.; Maciel, G. E. J. Phys. Chem. C 2008, $112,4315-4326$

(41) Howard, J. A. K.; Hoy, V. J.; Ohagan, D.; Smith, G. T. Tetrahedron 1996, 52, 12613-12622.

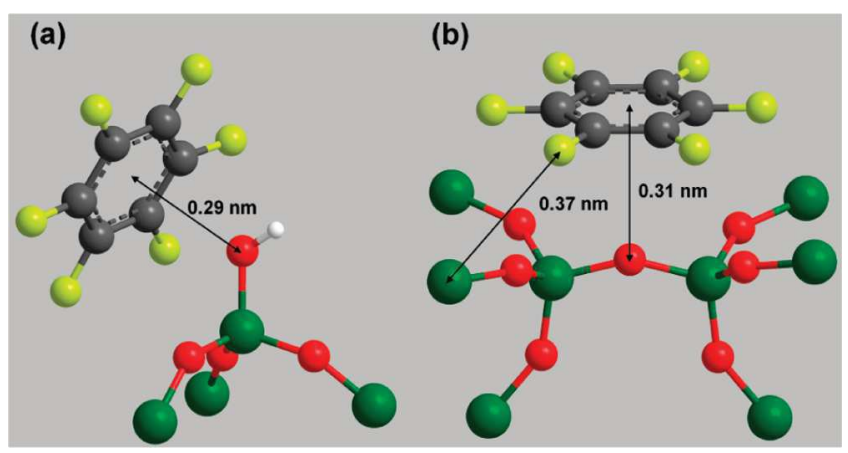

Figure 6. The optimized geometries of (a) $\mathrm{C}_{6} \mathrm{~F}_{6}-$ silanol and (b) $\mathrm{C}_{6} \mathrm{~F}_{6}-$ siloxane models. Silicon atoms are green, oxygen atoms are red, carbon atoms are gray, fluorine atoms are yellow, and hydrogen atom is white. Silanol hydrogen is the only one shown.

atoms. The structure of the Si-containing clusters was fixed during the optimization, except for the $\mathrm{OH}$ moieties of the silanol groups. The resulting geometries of the $\mathrm{C}_{6} \mathrm{~F}_{6}-$ silanol and $\mathrm{C}_{6} \mathrm{~F}_{6}$-siloxane environments are shown in Figure 6. In both structures, the center of the $\mathrm{C}_{6} \mathrm{~F}_{6}$ molecule appears to be located near the oxygen atom.

In the optimized $\mathrm{C}_{6} \mathrm{~F}_{6}-$ silanol geometry (Figure 6a), the distance between the oxygen atom and the center of mass of the aromatic ring and the interaction energy are calculated to be $d=0.29 \mathrm{~nm}$ and $\Delta E=-5.6 \mathrm{~kJ} / \mathrm{mol}$, respectively. Furthermore, five fluorine atoms of the $\mathrm{C}_{6} \mathrm{~F}_{6}$ are separated from the nearest silicon atom $\left(\mathrm{Q}^{3}\right.$ site) by more than the NMR estimate $(0.45 \mathrm{~nm})$. The hydrogen bonds between the surface silanols and the fluorine atoms do not seem to form on the silica surface, which is consistent with the previous studies of the $\mathrm{C}_{6} \mathrm{~F}_{6}-\mathrm{H}_{2} \mathrm{O}$ system. ${ }^{34}$ This result is not surprising, because fluorine that bonds to $\mathrm{sp}^{2}$ carbon is a poor hydrogen-bond acceptor. ${ }^{40,41}$ The geometry shown in Figure 6a suggests that on the "real" MSN surface the $\mathrm{C}_{6} \mathrm{~F}_{6}-$ silanol interaction can be affected by steric hindrance within the system. Furthermore, fast librational motions of the silanol $\mathrm{OH}$ moieties ${ }^{40}$ make it difficult for the aromatic ring and the silanol oxygen to interact with each other.

An optimized geometry of the $\mathrm{C}_{6} \mathrm{~F}_{6}$-siloxane system is shown in Figure 6b. In this case, the $\mathrm{C}_{6} \mathrm{~F}_{6}$ molecule lies flat above the siloxane oxygen at a distance of $d=0.31 \mathrm{~nm}$ with all fluorine atoms having one or more neighboring silicon atoms within $0.45 \mathrm{~nm}$. The computed interaction energy $(\Delta E=-11.0$ $\mathrm{kJ} / \mathrm{mol}$ ) is larger than that for the $\mathrm{C}_{6} \mathrm{~F}_{6}-$ silanol model. The mobility of the $\mathrm{C}_{6} \mathrm{~F}_{6}$ molecule under such conditions is expected to be limited.

The $\mathrm{C}_{6} \mathrm{~F}_{6}-$ siloxane system is further studied by theoretical calculation of the ${ }^{19} \mathrm{~F}$ chemical shift. The computation of the ${ }^{19} \mathrm{~F}$ chemical shift of an isolated $\mathrm{C}_{6} \mathrm{~F}_{6}$ molecule yielded -165.2 ppm, which is close to the reported value in the gas phase $(-167.75 \mathrm{ppm}){ }^{32}$ The ${ }^{19} \mathrm{~F}$ chemical shift of the $\mathrm{C}_{6} \mathrm{~F}_{6}$ molecule in the optimized $\mathrm{C}_{6} \mathrm{~F}_{6}$-siloxane system was calculated to be $-155.9 \mathrm{ppm}$ (average of all six fluorine atoms), which demonstrates that the interaction of $\mathrm{C}_{6} \mathrm{~F}_{6}$ molecules with the siloxane oxygen has a deshielding effect on the ${ }^{19} \mathrm{~F}$ nuclei compared to the $\mathrm{C}_{6} \mathrm{~F}_{6}$ in the gas phase. This deshielding effect is in agreement with ${ }^{19} \mathrm{~F}$ NMR data, although its size exceeds the experimental value of $\sim 3 \mathrm{ppm}$. However, on the "real" surface, the aromatic ring of the PFP-p species is not located in the geometry where it maximally interacts with the siloxane oxygen because of the constraints imposed by the propyl chain, as well as the physical topology and chemical structure (e.g., concentration of silanol 

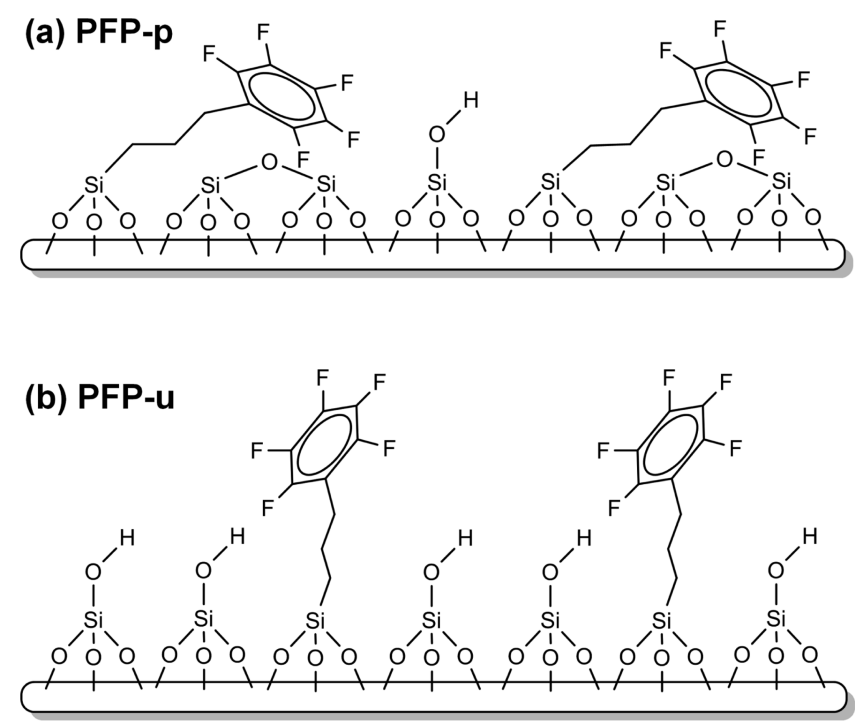

Figure 7. The schematic representation of (a) PFP-p and (b) PFP-u functional groups on the silica surface. The aromatic rings of PFP-p interact with the siloxane oxygen, whereas those in PFP-u are located further from the surface and are more mobile.

groups) of the MSN surface. Besides, even the PFP-u species are not truly isolated in the narrow pore of the MSN materials. Thus, the difference in ${ }^{19} \mathrm{~F}$ chemical shifts between PFP-p and PFP-u is likely to be smaller than that calculated for the $\mathrm{C}_{6} \mathrm{~F}_{6}$ - siloxane model.

The theoretical calculations suggest that a feasible model representing the PFP-p species is one in which the aromatic ring of the PFP group is located above the siloxane oxygen in parallel to the MSN surface (Figure 7a). This geometry is consistent with the following NMR results: (1) the ${ }^{19} \mathrm{~F}$ nuclei in PFP-p groups are located within $\sim 0.45 \mathrm{~nm}$ from the nearest $\mathrm{Q}^{3}$ and $\mathrm{Q}^{4}$ silicon sites, (2) the mobility of the ring is restricted compared to PFP-u, (3) the ${ }^{19} \mathrm{~F}$ nuclei of the PFP-p are deshielded by the interaction with silica. Due to the abovementioned steric constraints, only a fraction of the PPF groups can be located above the siloxane oxygen as PFP-p, whereas the others are confined to PFP-u positions. The most likely structure of PFP-u is shown in Figure 7b. We should emphasize that the designation of PFP-u molecules as "upright" does not imply that they assume rigid vertical positions on the surface. The NMR data showed reduced ${ }^{19} \mathrm{~F}$ line width and slower transverse relaxation in $\mathrm{PFP}-\mathrm{u}$, most likely as a result of librational flips or rotations. Whereas this mobility may slow down the ${ }^{19} \mathrm{~F}-{ }^{29} \mathrm{Si} \mathrm{CP}$ process, the absence of resonances representing PFP-u in the ${ }^{19} \mathrm{~F}-{ }^{29} \mathrm{Si}$ HETCOR spectrum and the change in ${ }^{19} \mathrm{~F}$ chemical shifts demonstrate that these species are more distant from the MSN surface than PFP-p.

\section{Conclusion}

The NMR results and the theoretical calculations presented in this study showed that the PFP-MSN material hosts two types of covalently bound PFP species with similar bond topologies but different conformations: the PFP-p groups with the aromatic rings located above the surface siloxanes and the PFP-u molecules in the roughly upright position. The fundamental understanding of the silica surface provided by this investigation can be used to enhance the catalytic performance in a predictable way. The interaction energy calculated for the $\mathrm{C}_{6} \mathrm{~F}_{6}-$ siloxane model system is larger than the adsorption energy of water molecules to dehydrated surface of a mesoporous silica material at low vapor pressure. ${ }^{42}$ Since surface siloxanes of MSN materials are easily hydrolyzed to form silanols by water adsorption even at room temperature, ${ }^{43,44}$ enhancing the hydrophobic character of these materials by protecting the siloxanes from water should be beneficial for their catalytic applications.

Indeed, in a forthcoming publication we will report on the design of a bifunctional MSN-based catalyst containing an acidic catalytic site and PFP functionalities for an esterification reaction. The presence of PFP improved considerably the catalytic performance, which is attributed to efficient expulsion of the byproduct (water) from the PFP-coated mesochannels. Further experimental and theoretical investigations are underway to describe the behavior of the PFP functional groups on the MSN surfaces in the presence of solvents.

Acknowledgment. This research was supported at the Ames Laboratory by the U.S. Department of Energy, Office of Basic Energy Sciences, under Contract No. DE-AC02-07CH11358. We would also like to thank the U.S. Department of Energy, Office of Energy Efficiency and Renewable Energy (Grant No. DE-FG260NT08854), for financial support.

\section{JA105007B}

(42) Cauvel, A.; Brunel, D.; DiRenzo, F.; Garrone, E.; Fubini, B. Langmuir 1997, 13, 2773-2778

(43) Matsumoto, A.; Sasaki, T.; Nishimiya, N.; Tsutsumi, K. Langmuir 2001, 17, 47-51.

(44) Matsumoto, A.; Tsutsumi, K.; Schumacher, K.; Unger, K. K. Langmuir 2002, 18, 4014-4019. 


\title{
High-Throughput Analysis of Algal Crude Oils Using High Resolution Mass Spectrometry
}

\author{
Young Jin Lee $\cdot$ Rachael C. Leverence . \\ Erica A. Smith • Justin S. Valenstein • \\ Kapil Kandel · Brian G. Trewyn
}

Received: 11 November 2012/ Accepted: 3 January 2013

(C) AOCS (outside the USA) 2013

\begin{abstract}
Lipid analysis often needs to be specifically optimized for each class of compounds due to its wide variety of chemical and physical properties. It becomes a serious bottleneck in the development of algae-based next generation biofuels when high-throughput analysis becomes essential for the optimization of various process conditions. We propose a high-resolution mass spectrometry-based high-throughput assay as a 'quick-and-dirty' protocol to monitor various lipid classes in algal crude oils. Atmospheric pressure chemical ionization was determined to be most effective for this purpose to cover a wide range of lipid classes. With an autosampler-LC pump set-up, we could analyze algal crude samples every one and half minutes, monitoring several lipid species such as TAG, DAG, squalene, sterols, and chlorophyll $a$. High-mass resolution and high-mass accuracy of the orbitrap mass analyzer provides confidence in the identification of these lipid compounds. MS/MS and MS3 analysis could be performed in parallel for further structural information, as demonstrated for TAG and

Electronic supplementary material The online version of this article (doi:10.1007/s11745-013-3757-7) contains supplementary material, which is available to authorized users.

Y. J. Lee $(\varangle)$ - R. C. Leverence - E. A. Smith .

J. S. Valenstein · K. Kandel · B. G. Trewyn

U.S.-DOE Ames Laboratory, Ames, IA 50011, USA

e-mail: yjlee@iastate.edu

Y. J. Lee · E. A. Smith · J. S. Valenstein · K. Kandel

B. G. Trewyn

Department of Chemistry, Iowa State University,

Ames, IA 50011, USA

Present Address:

B. G. Trewyn

Department of Chemistry and Geochemistry,

Colorado School of Mines, Golden, CO 80401, USA

DAG. This high-throughput method was successfully demonstrated for semi-quantitative analysis of algal oils after treatment with various nanoparticles.

Keywords Analytical chemistry - Analytical techniques · Mass spectrometry (MS)

\begin{tabular}{|c|c|}
\hline Abbreviation & \\
\hline APCI & Atmospheric pressure chemical ionization \\
\hline AP-MSN-3 & $\begin{array}{l}\text { Aminopropyl functionalized mesoporous } \\
\text { silica nanoparticles with } 3 \mathrm{~nm} \text { pore size }\end{array}$ \\
\hline AP-MSN-5 & $\begin{array}{l}\text { Aminopropyl functionalized mesoporous } \\
\text { silica nanoparticles with } 5 \mathrm{~nm} \text { pore size }\end{array}$ \\
\hline AP-MSN-10 & $\begin{array}{l}\text { Aminopropyl functionalized mesoporous } \\
\text { silica nanoparticles with } 10 \mathrm{~nm} \text { pore size }\end{array}$ \\
\hline APPI & Atmospheric pressure photoionization \\
\hline CHCA & a-Cyano-4-hydroxycinnamic acid \\
\hline CID & Collision-induced dissociation \\
\hline CTAB & Cetyl trimethylammonium bromide \\
\hline DAG & Diacylglycerols \\
\hline ESI & Electrospray ionization \\
\hline FFA & Free fatty acids \\
\hline FTICRMS & $\begin{array}{l}\text { Fourier transform ion cyclotron resonance } \\
\text { mass spectrometer }\end{array}$ \\
\hline GC & Gas chromatography \\
\hline HPLC & High performance liquid chromatography \\
\hline HRMS & High resolution mass spectrometry \\
\hline MALDI & Matrix-assisted laser desorption ionization \\
\hline MS & Mass spectrometry \\
\hline MS/MS & Tandem mass spectrometry \\
\hline MS3 & $\begin{array}{l}\text { MS/MS/MS, triple stage tandem mass } \\
\text { spectrometry }\end{array}$ \\
\hline MSN-10 & $\begin{array}{l}\text { Mesoporous silica nanoparticles with } \\
10 \mathrm{~nm} \text { pore size }\end{array}$ \\
\hline SPE & Solid phase extraction \\
\hline
\end{tabular}

\section{Abbreviations}

APCI Atmospheric pressure chemical ionization

AP-MSN-3 Aminopropyl functionalized mesoporous

AP-MSN-5 Aminopropyl functionalized mesoporous

AP-MSN-10 Aminopropyl functionalized mesoporous

APPI Atmospheric pressure photoionization

CHCA $\quad \alpha$-Cyano-4-hydroxycinnamic acid

CID Collision-induced dissociation

CTAB Cetyl trimethylammonium bromide

DAG Diacylglycerols

ESI

Electrospray ionization

FFA Free fatty acids

FTICRMS Fourier transform ion cyclotron resonance mass spectrometer

GC Gas chromatography

HPLC High performance liquid chromatography

HRMS High resolution mass spectrometry

MALDI Matrix-assisted laser desorption ionization

MS Mass spectrometry

MS/MS Tandem mass spectrometry

spectrometry

Solid phase extraction
\end{abstract}


TAG

TEOS

Triacylglycerols

Tetraethylorthosilicate

\section{Introduction}

Biofuel has been gaining tremendous interest in recent years due to the high cost of petroleum and environmental impact of burning fossil fuels as well as its role in energy security and domestic economy $[1,2]$. The current biodiesel and bioethanol, so-called first generation biofuels, are derived from sugars and oil seeds in crop plants. As such, they are not sustainable due to the use of a large area of farmland [3]. Second generation biofuels such as cellulosic bioethanol or pyrolysis bio-oils are gaining in popularity since they are derived from non-food feedstocks and use non-agricultural land. Microalgae are among the very attractive feedstock for third generation biofuels. They utilize less land and produce more biomass than seasonal plant crops due to efficient use of solar energy [1]. Additionally, they require much less water and nutrients from the soil [2].

Microalgae, however, have many obstacles on their path to large-scale biofuel production. Subsequently, there are urgent demands for scientific breakthroughs in the strategy for harvesting and processing of algal biomass. Developing a new technology also commands substantial efforts on the analytical chemistry side. Specifically, testing and optimizing new technology for next generation biofuels require high-throughput analysis because of the many variables that need to be optimized. This is especially the case for microalgae compared to other biomass feedstock because the optimization is often species and pretreatment dependent due to the significant differences in cell membranes and lipid compositions [2].

The analysis of microalgal lipids has been largely focused on triacylglycerols (TAG). The traditional analytical protocol to evaluate TAG is gas chromatography (GC) [4-7]. Major drawbacks of using GC or GC-MS include multiple sample preparation steps and no direct TAG analysis. Direct analysis of intact TAG species has been achieved with electrospray ionization mass spectrometry (ESI-MS) using lithium [8] or ammonium [9] as a cationization reagent as well as matrix-assisted laser desorption ionization (MALDI)-MS [10]. For better speciation of algal TAG, high performance liquid chromatography (HPLC) has been employed with ESI $[9,11]$ or atmospheric pressure chemical ionization (APCI) [12]. Many of these analyses, however, do not include the analysis of other lipid components that might be also critical in new process development.
High-resolution mass spectrometry (HRMS) has become an invaluable tool for the characterization of algal lipids. HRMS identified over 100 distinct TAG in six algae strains using a standalone bench-top orbitrap mass spectrometer with HPLC separation [9]. HRMS was also used to characterize algal polar lipids using HPLC-ESI-linear ion trap (LTQ) coupled with 14.5 T Fourier transform ion cyclotron resonance mass spectrometer (FTICR-MS) [11]. Ultrahigh mass resolution of FTICR-MS determined the lipid elemental composition from accurate mass and isotopic fine structure at baseline resolution [11].

Mesoporous silica nanoparticle (MSN) materials synthesized with surfactant micelle-template have a high surface area and pore volume that lead to a wide range of applications, such as catalysts [13], sensors [14], and delivery vessels [15]. MSN is particularly useful because the pore size can be easily controlled and the surface can be functionalized with organic groups. Recently, we utilized the versatility of MSN to synthesize various MSN materials and screen the materials that can specifically sequester free fatty acids (FFA) from algal crude oils [16]. However, the analytical protocol was limited to GC-MS analysis in the previous study, which is not only laborious but also incapable of characterizing intact TAG species.

Here, we propose a high-throughput assay to analyze algal crude oils using orbitrap HRMS without LC separation. Our approach is not intended to be comprehensive for all TAG or other lipid species, but rather focused on highthroughput comparison of major lipid compounds for various processing conditions. In this approach, each sample could be analyzed every one and half minute with an autosampler-LC pump set-up for various classes of non- or partially polar lipids. As the first step of demonstrating its utility, the proposed approach is applied to algal crude oils, much less complex than an algal extract. This assay was successfully applied for a semi-quantitative comparison of algal crude oils after treatment with MSN materials for the sequestration of certain classes of lipids.

\section{Materials and Methods}

\section{Chemicals}

A simulated algal oil and crude algal oil were used for the current study. The simulated algal oil was composed of octadecanoic acid, palmitic acid, glyceryl tripalmitate, squalene and ergosterol ( $400 \mu \mathrm{M}$ for each) dissolved in $50 \%$ methanol in toluene. The crude algal oil originated from Nannochloropsis salina and supplied by Solix Biofuels, Inc. (Fort Collins, CO, USA). All other chemicals were of the highest grade commercially available. 
Synthesis of Mesoporous Silica Nanoparticles

The synthesis of 10-nm pore sized MSNs with and without aminopropyl functionalization (MSN-10 and AP-MSN-10) is described elsewhere [16]. The synthesis of the other MSN is as follows.

\section{Synthesis of AP-MSN-3}

Cetyltrimethylammonium bromide (CTAB, $1.0 \mathrm{~g})$ was dissolved in nanopure water (480 g), followed by the addition of $\mathrm{NaOH}$ solution $(2.0 \mathrm{M}, 3.5 \mathrm{~mL})$. The mixture was heated to $80{ }^{\circ} \mathrm{C}$ for $1 \mathrm{~h}$. To this clear solution, tetraethylorthosilicate (TEOS) (4.7 g) was added drop wise at the rate of $1 \mathrm{~mL} \mathrm{~min}^{-1}$ followed by drop-wise addition of 3-aminopropyltrimethoxysilane $\left(2.56 \times 10^{-3} \mathrm{~mol}, 0.46 \mathrm{~g}\right)$. The reaction mixture was stirred vigorously at $80{ }^{\circ} \mathrm{C}$ for $2 \mathrm{~h}$ and then filtered to yield a white solid. The as-synthesized material was washed with copious amounts of water and methanol, and then dried under a vacuum. The CTAB surfactant was removed via Soxhlet extraction with methanol for $24 \mathrm{~h}$, followed by drying overnight under a vacuum.

\section{Synthesis of AP-MSN-5}

The AP-MSN-5 was prepared following the same procedure as described above, except for the initial step which involved adding the pore expander agent mesitylene $(7.0 \mathrm{~mL})$ to the original CTAB solution.

Sequestration of Algal Oils by MSN Treatment

Four MSN materials (AP-MSN-3, AP-MSN-5, AP-MSN10, MSN-10) were used to adsorb lipids from crude microalgal oil. Each MSN material (100 mg) was added to $200 \mathrm{mg}$ of microalgal oil in $10 \mathrm{~mL}$ of hexanes and mixed for $2 \mathrm{~h}$. After $2 \mathrm{~h}$, the suspension was centrifuged and the supernatant was used for analyses.

\section{Mass Spectrometric Data Acquisition}

The crude algal oil samples were analyzed with a linear ion trap-orbitrap hybrid mass spectrometer (LTQ-Orbitrap Discovery, Thermo Scientific, San Jose, CA, USA) using various ionization methods; including ESI, APCI, atmospheric pressure photoionization (APPI), and MALDI. The orbitrap analyzer was used for the mass spectral analysis with a mass resolution of 30,000 at $\mathrm{m} / \mathrm{z}$ 400. Mass accuracy was $5 \mathrm{ppm}$ or below with external calibration for all the spectra. Internal calibration was made for APCI and APPI data with the most abundant peak (i.e., DAG fragment of $\left[\mathrm{C}_{34} \mathrm{H}_{64} \mathrm{O}_{4}+\mathrm{H}\right]^{+}$at $\mathrm{m} / z$ 549.4877), which resulted in a mass error of 3 ppm or below.
The crude algal oil was diluted to $0.01 \%$ (v/v) with $50 \%$ methanol in toluene for mass spectrometry analysis and injected into the mass spectrometer either manually or via an autosampler. The manual injection of crude algal oil was performed using a sample loop $(10 \mu \mathrm{L})$ and a divert valve at a solvent flow of $25 \mu \mathrm{L} \min ^{-1}$ (ESI) or $75 \mu \mathrm{L}$ $\min ^{-1}$ (APCI/APPI) with a syringe pump. An autosamplerLC set-up (HTC PAL, CTC Analytics, Switzerland; Paradigm MS4, Michron BioResources, Auburn, CA, USA) was also used for the automatic injection of crude algal oils.

In ESI analysis, the capillary voltage and temperature were set at $33 \mathrm{~V}$ and $200{ }^{\circ} \mathrm{C}$, respectively. The sheath gas flow rate was 1 (arb) and the tube lens voltage was $83 \mathrm{~V}$. In APCI and APPI, the vaporizer heater was operated at 300-350 ${ }^{\circ} \mathrm{C}$ and the heated capillary set to $175^{\circ} \mathrm{C}$. Sheath and auxiliary gases were set to 50 (arb) and 5 (arb), respectively, and tube lens voltage was set to $29 \mathrm{~V}$. The corona current was set to $5 \mu \mathrm{A}$ in APCI. A vacuum ultraviolet lamp (PhotoMate, 10.0/10.2 eV, Syagen, Tustin, CA, USA) was used for APPI. In a quantitative study for cholesterol, cholesterol standard was added to $0.01 \%$ crude algal oil at a final concentration of 50-200 $\mu \mathrm{M}$. For MALDI-MS analysis, the instrument was re-configured with a MALDI source. A custom-installed Nd:YAG laser (UVFQ, Elforlight, UK) was used at a laser power of 1-3 $\mu \mathrm{J}$ per pulse. All algae oil samples were analyzed in the positive ion mode. Collision-induced dissociation (CID) was performed in the LTQ with an isolation window of $1.8 \mathrm{Da}$ and collision energy of $35-45 \%$.

GC-MS was performed on an Agilent 7890A gas chromatograph equipped with a $6975 \mathrm{C}$ MS in electron ionization mode. The crude algal oil was diluted to $2 \%$ (v/v) in $50 \% \mathrm{MeOH}$ in toluene. Twenty microliters was dried and silylated with $\mathrm{N}, \mathrm{O}$-bis (trimethylsilyl) trifluoroacetamide with trimethylchlorosilane (100 $\mu \mathrm{L}$; Sigma, St. Louis, MO, USA) by heating at $65^{\circ} \mathrm{C}$ for $30 \mathrm{~min}$, then evaporated with nitrogen. After dilution in $1 \mathrm{~mL}$ of toluene, one microliter of the sample was injected into the GC-MS with a HP-5 ms $5 \%$ Phenyl Methyl Silox column $(250 \mu \mathrm{m}$ i.d., $30 \mathrm{~m}, 0.25 \mu \mathrm{m}$ film thickness) at an injector temperature of $325^{\circ} \mathrm{C}$ in splitless mode and a helium flow rate of

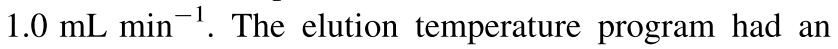
initial temperature of $80{ }^{\circ} \mathrm{C}$ and then linearly ramped to $280{ }^{\circ} \mathrm{C}$ at $20^{\circ} \mathrm{C} \min ^{-1}$ and finally to $320^{\circ} \mathrm{C}$ at $10{ }^{\circ} \mathrm{C}$ $\min ^{-1}$. NIST 08 Mass Spectral Library was used to identify the compounds.

\section{Results and Discussion}

Our initial focus was to find the most effective ionization method that can characterize various classes of lipid 
species in algal oils. We were particularly interested in five major lipids: TAG, sterols, terpenes, chlorophyll $a$, and FFA. We found difficulty in analyzing FFA with atmospheric pressure ionization (i.e., APCI, APPI, and ESI) or MALDI, particularly due to the common contamination from pump oils or evaporation inside the MALDI vacuum chamber. In addition, their analysis is most effective in negative ion mode while the rest can be ionized almost exclusively in positive ion mode. Accordingly, subsequent efforts were made for the other lipids in positive ion mode because FFA can be routinely and effectively analyzed in the current GC-MS method.

Figure 1 compares orbitrap MS spectra obtained with four different ionization methods for algal crude oils. Each ionization method has its own pros and cons. MALDI provides the highest throughput, taking only a few seconds per sample for the data acquisition, but the presence of chlorophyll $a$ completely suppresses TAG and DAG signals [10], the most important lipid classes, and also significantly suppresses other compounds. A few matrixes were attempted, but we could not find a good matrix that can provide sufficient ion signals for TAG in the presence of chlorophyll. Danielewicz and coworkers [10] successfully analyzed TAG after the removal of chlorophyll with solid phase extraction; however, it required additional steps and led to the loss of other lipids along with chlorophyll.

Electrospray ionization is the best method for the analysis of TAG because there is no apparent ion suppression by chlorophyll (vs. MALDI) and no fragmentation (vs. APPI or APCI). With potassium acetate as an additive in the solution, TAG and DAG could be detected as a potassium ion adduct without any fragmentation. However, because of ion suppression, ESI cannot efficiently ionize non-polar lipids, such as terpene (e.g., squalene) or sterols (e.g., cholesterol), when they are co-present with other algal lipids. Hence, ESI was not considered to be a good ionization method for the monitoring of those lipids.

Both APPI and APCI can ionize sterols equally well, but APPI ionization efficiency is low for terpene. As shown in Fig. 1, we could barely detect squalene ion signals with APPI. Furthermore, ion signals are subjected to contamination in APPI, especially by plasticizers. One limitation in both APCI and APPI is the significant fragmentation of TAG species. The base peak of the spectra $(\mathrm{m} / \mathrm{z} 549.4886)$ is assigned to the chemical composition of $\mathrm{C}_{35} \mathrm{H}_{65} \mathrm{O}_{4}$ $(\Delta m=1.6 \mathrm{ppm})$ and corresponds to a fragment ion of TAG containing C16:0 and C16:1 fatty acids. However, we could still observe intact TAG and DAG species in significant intensities, along with cholesterol, ergosterol, stigmasterol, squalene, $\alpha$-tocopherol, and chlorophyll $a$. Overall, we decided APCI is best suited for the rapid monitoring of lipid species in algal oils.
A similar trend was found for a simulated solution composed of octadecanoic acid, palmitic acid, glyceryl tripalmitate, ergosterol, and squalene (Supplementary Figure 1). A few minor differences are noteworthy. The TAG was composed of fully saturated fatty acids in all three locations (C16:0/C16:0/C16:0) and easily dissociated insource to DAG fragments in the APCI or APPI condition. We did not observe any intact C16 TAG in APPI or APCI, while intact C16 TAG was apparent in ESI as a potassium ion adduct. However, as expected, no peak for ergosterol or squalene could be seen in ESI unlike in APCI or APPI. Squalene was now observed in APPI, mostly as a molecular radical ion. With no chlorophyll in the solution, C16 TAG was also seen in the MALDI-MS spectrum.

High-resolution mass spectrometry is a key to the high throughput assay we are developing. With no chromatographic separation, we rely on high-resolution mass spectrometric separation to assign lipid species. It is particularly important in the low $\mathrm{m} / \mathrm{z}$ range where nonlipid-origin contamination might be present in the sample that cannot be distinguished in low-resolution MS. For example, squalene at $\mathrm{m} / \mathrm{z} 411.3991\left(\left[\mathrm{C}_{30} \mathrm{H}_{50}+\mathrm{H}\right]^{+}\right)$ has an unknown peak nearby at $\mathrm{m} / \mathrm{z} \quad 411.3255$ $\left(\left[\mathrm{C}_{28} \mathrm{H}_{42} \mathrm{O}_{2}+\mathrm{H}\right]^{+}\right)$. Even though the ion signal is typically less than $10 \%$ of squalene, it might be incorrectly included in the squalene ion signal in low-resolution MS. In addition, HRMS allows direct chemical composition analysis of each compound, which is essential in the current approach. It should be noted our method is not comprehensive compared to some other approaches available. We cannot distinguish structural isomers with HRMS alone, which can be performed with chromatographic separations and/or tandem mass spectrometric analysis. However, HRMS provides sufficient selectivity for major lipids and we can supplement the structural information using MS/MS and/or MS3 when necessary.

Table 1 shows a list of algal lipids identified in an APCI-orbitrap MS spectrum shown in Fig. 1. A total of 48 lipid species were assigned based on accurate masses (mass error of $4 \mathrm{ppm}$ or less). In addition to the speciation of 32 TAG and 10 DAG, we characterized 3 sterols, squalene, $\alpha$ tocopherol, and chlorophyll $a$ using this approach. The presence of sterols, squalene, and $\boldsymbol{\alpha}$-tocopherol was also confirmed in GC-MS analysis. High-resolution MS provides a major role in differentiating intact DAG and DAG fragments from TAG. The intact DAG molecules have five oxygens and can be easily differentiated from DAG fragments produced by in-source fragmentation of TAG that have four oxygens.

One should note the relative ion abundance between each TAG species does not quantitatively represent their actual relative abundance. It is well known that TAG with highly unsaturated fatty acyl chains have higher APCI 
Fig. 1 Orbitrap MS spectra of algal crude oils ionized with ESI, APCI, APPI, and MALDI. Most ions are detected as protonated, except potassium adduct of TAG and DAG in ESI. Chlorophyll $a$ is detected as [chlorophyll $\left.a-\mathrm{Mg}^{2+}+2 \mathrm{H}\right]^{+}$ (ChlA) or as an oxidation product, [chlorophyll $a+\mathrm{O}-$ $\left.\mathrm{Mg}^{2+}+2 \mathrm{H}\right]^{+}($ChlA $*$ ). Sterols are detected with a water-loss, $\left[\mathrm{M}-\mathrm{H}_{2} \mathrm{O}+\mathrm{H}\right]^{+}$, labeled as $\mathrm{C}, \mathrm{E}$, and St for cholesterol, ergosterol, and stigmasterol, respectively. Squalene and $\alpha$-tocopherol are labeled as Sq and T, respectively, and chlorophyll $a$ fragments are labeled as ChlAF. $\alpha$-tocopherol is detected as a protonated ion in APCI, $[\mathrm{M}+\mathrm{H}]^{+}$, and as a radical ion in APPI, $\mathrm{M}^{+}$

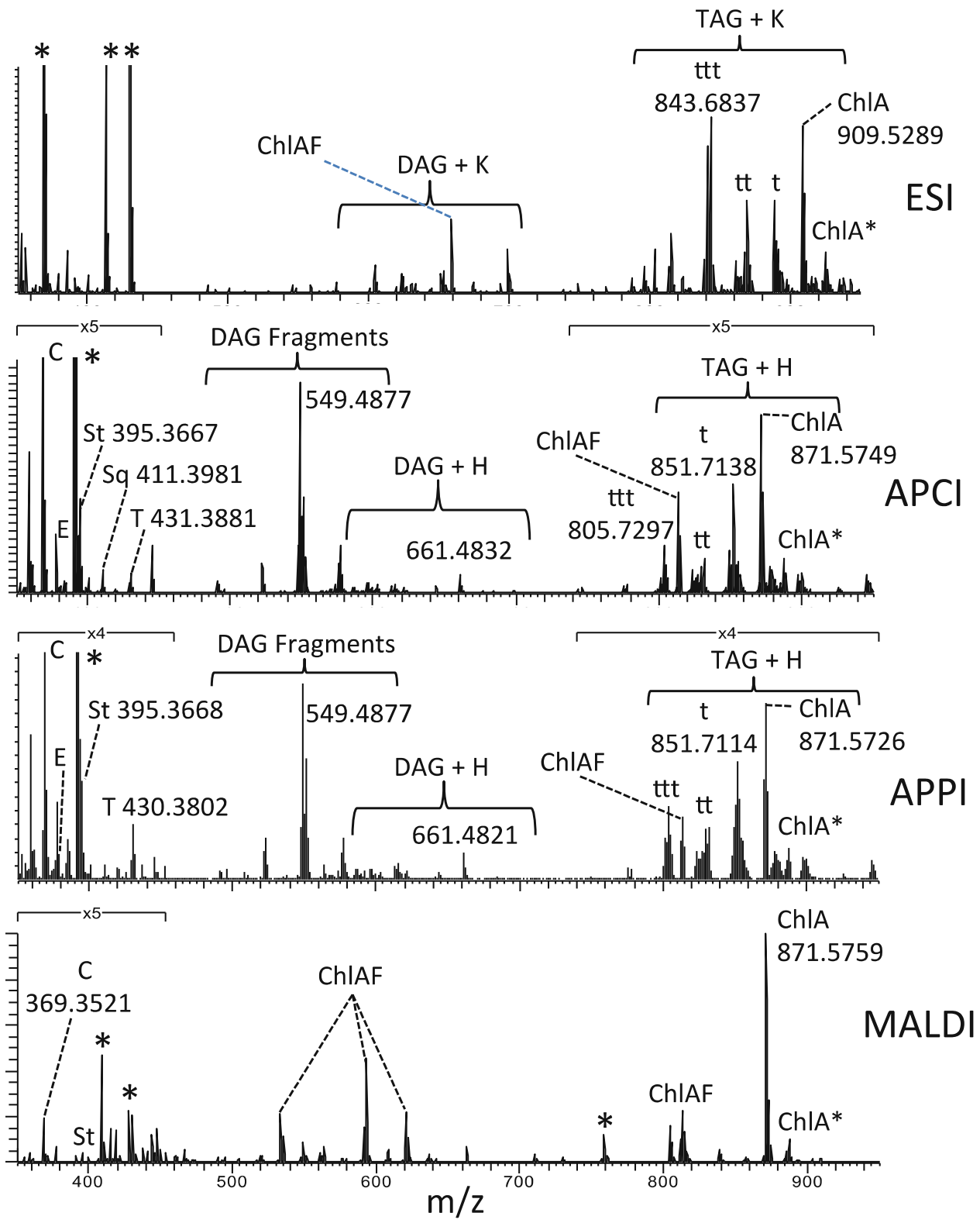

ionization efficiency [17]. For example, TAG species labeled as $t, t t$, and $t t t$ in Fig. 1, corresponding to C16:0/ C16:1/C20:5, C16:0/C16:1/C18:1, and C16:0/C16:0/C16:1 in fatty acyl compositions, show different relative ion abundances between ESI and APCI. The least unsaturated TAG ( $t t t)$ has the highest abundance in ESI, while the most unsaturated TAG $(t)$ has the highest abundance in APCI.

Further MS/MS and MS3 analyses were made for some compounds, mostly TAG and DAG, to further support their structural assignments. Supplementary Table 1 shows the summary of MS/MS and MS3 of TAG species obtained with the linear ion trap of the instrument to assign the fatty acid moiety. The major fragmentations in MS/MS of TAG are the loss of water and RCOOH. The MS3 was made for the loss of RCOOH in a few major TAG and resulted in the products of $\left[\mathrm{R}^{\prime} \mathrm{CO}\right]^{+},\left[\mathrm{R}^{\prime} \mathrm{CO}-\mathrm{H}_{2} \mathrm{O}\right]^{+},\left[\mathrm{R}^{\prime} \mathrm{COO}+58\right]^{+}$, and $\left[\mathrm{R}^{\prime} \mathrm{COO}+58-\mathrm{H}_{2} \mathrm{O}\right]^{+}$as assigned by Byrdwell and Neff [18]. The majority of TAG and DAG contain highly polyunsaturated fatty acids (PUFA), tentatively assigned as eicosapentaenoic acid (EPA; C20:5), linolenic acid (C18:3) and arachidonic acid (ARA; C20:4). The other major fatty acid present in the algal oil was C16 with up to three unsaturated sites. Khozin-Goldberg and Boussiba [19] reported that palmitic (16:0), palmitoleic (16:1) and EPA (20:5) are the three main fatty acids observed in the genus $N$. salina, in good accordance with our findings.

As the plausibility of characterizing various lipid species using APCI-HRMS had been verified, the next analytical parameters to be tested were throughput and quantification. To introduce samples into a MS system, we used both 
Table 1 Summary of identified lipids in algae crude oil by APCI-HRMS

\begin{tabular}{|c|c|c|c|c|c|}
\hline & $m / z$ & Assignment & Mass error (ppm) & Relative intensity & Supporting information \\
\hline \multirow[t]{32}{*}{ Triacylglycerols } & 775.6822 & $\mathrm{C} 16: 1 / \mathrm{C} 16: 1 / \mathrm{C} 14: 0$ & 1.6 & 4.7 & MS2 \\
\hline & 777.6982 & $\mathrm{C} 16: 0 / \mathrm{C} 16: 1 / \mathrm{C} 14: 0$ & 2.1 & 5.8 & MS2 \\
\hline & 795.6473 & $\mathrm{C} 16: 2 / \mathrm{C} 16: 2 / \mathrm{C} 16: 2$ & -3.0 & 1.0 & \\
\hline & 797.6674 & $\mathrm{C} 16: 2 / \mathrm{C} 16: 2 / \mathrm{C} 16: 1$ & 2.6 & 2.2 & \\
\hline & 799.6816 & $\mathrm{C} 16: 2 / \mathrm{C} 16: 1 / \mathrm{C} 16: 1$ & 0.8 & 3.1 & MS2 \\
\hline & 801.6980 & $\mathrm{C} 14: 0 / \mathrm{C} 16: 1 / \mathrm{C} 18: 2$ & 1.7 & 11.0 & MS2 \\
\hline & 803.7140 & $\mathrm{C} 14: 0 / \mathrm{C} 18: 2 / \mathrm{C} 16: 0$ & 2.1 & 31.7 & MS2 \\
\hline & 805.7297 & $\mathrm{C} 16: 0 / \mathrm{C} 16: 0 / \mathrm{C} 16: 1$ & 2.2 & 18.7 & MS2 \\
\hline & 823.6826 & $\mathrm{C} 14: 0 / \mathrm{C} 16: 1 / \mathrm{C} 20: 5$ & 2.0 & 21.5 & $\mathrm{MS} 2 / \mathrm{MS} 3$ \\
\hline & 825.6973 & $\mathrm{C} 16: 1 / \mathrm{C} 16: 1 / \mathrm{C} 18: 3$ & 0.9 & 16.1 & \\
\hline & 827.7134 & $\mathrm{C} 16: 0 / \mathrm{C} 16: 1 / \mathrm{C} 18: 3$ & 1.4 & 15.7 & MS2 \\
\hline & 829.7295 & $\mathrm{C} 16: 0 / \mathrm{C} 16: 0 / \mathrm{C} 18: 3$ & 1.9 & 20.4 & MS2 \\
\hline & 831.7451 & $\mathrm{C} 16: 0 / \mathrm{C} 16: 1 / \mathrm{C} 18: 1$ & 1.9 & 23.3 & $\mathrm{MS} 2 / \mathrm{MS} 3$ \\
\hline & 849.6983 & $\mathrm{C} 16: 1 / \mathrm{C} 16: 1 / \mathrm{C} 20: 5$ & 2.0 & 39.2 & MS2/MS3 \\
\hline & 851.7138 & $\mathrm{C} 16: 0 / \mathrm{C} 16: 1 / \mathrm{C} 20: 5$ & 1.8 & 100.0 & MS2/MS3 \\
\hline & 853.7286 & $\mathrm{C} 16: 0 / \mathrm{C} 16: 1 / \mathrm{C} 20: 4$ & 0.8 & 50.0 & MS2 \\
\hline & 855.7449 & $\mathrm{C} 16: 0 / \mathrm{C} 16: 0 / \mathrm{C} 20: 4$ & 1.5 & 26.3 & \\
\hline & 875.7140 & $\mathrm{C} 16: 1 / \mathrm{C} 18: 3 / \mathrm{C} 20: 4$ & 1.9 & 9.1 & MS2 \\
\hline & 877.7298 & $\mathrm{C} 16: 1 / \mathrm{C} 18: 1 / \mathrm{C} 20: 5$ & 2.1 & 21.8 & $\mathrm{MS} 2 / \mathrm{MS} 3$ \\
\hline & 879.7450 & $\mathrm{C} 16: 0 / \mathrm{C} 18: 1 / \mathrm{C} 20: 5$ & 1.6 & 22.7 & MS2 \\
\hline & 881.7603 & $\mathrm{C} 16: 0 / \mathrm{C} 18: 0 / \mathrm{C} 20: 5$ & 1.2 & 12.7 & \\
\hline & 897.6988 & C16:1/C20:5/C20:5 & 2.4 & 17.9 & MS2 \\
\hline & 899.7138 & $\mathrm{C} 16: 0 / \mathrm{C} 20: 5 / \mathrm{C} 20: 5$ & 1.7 & 19.1 & MS2/MS3 \\
\hline & 901.7285 & $\mathrm{C} 16: 0 / \mathrm{C} 20: 5 / \mathrm{C} 20: 4$ & 0.7 & 10.3 & \\
\hline & 903.7442 & $\mathrm{C} 16: 0 / \mathrm{C} 20: 4 / \mathrm{C} 20: 4$ & 0.7 & 5.4 & \\
\hline & 905.7603 & $\mathrm{C} 16: 0 / \mathrm{C} 20: 4 / \mathrm{C} 20: 3$ & 1.2 & 3.3 & \\
\hline & 923.7136 & $\mathrm{C} 18: 2 / \mathrm{C} 20: 5 / \mathrm{C} 20: 5$ & 1.4 & 2.3 & \\
\hline & 925.7302 & $\mathrm{C} 18: 1 / \mathrm{C} 20: 5 / \mathrm{C} 20: 5$ & 2.4 & 7.7 & \\
\hline & 927.7439 & $\mathrm{C} 18: 0 / \mathrm{C} 20: 5 / \mathrm{C} 20: 5$ & 0.3 & 3.9 & \\
\hline & 929.7570 & $\mathrm{C} 18: 0 / \mathrm{C} 20: 5 / \mathrm{C} 20: 4$ & -2.4 & 0.9 & \\
\hline & 945.6986 & $\mathrm{C} 20: 5 / \mathrm{C} 20: 5 / \mathrm{C} 20: 5$ & 2.1 & 18.6 & MS2 \\
\hline & 947.7129 & $\mathrm{C} 20: 5 / \mathrm{C} 20: 5 / \mathrm{C} 20: 3$ & 0.7 & 11.5 & \\
\hline \multirow[t]{10}{*}{ Diacylglycerols } & 585.4511 & $\mathrm{C} 16: 3 / \mathrm{C} 18: 3 / \mathrm{OH}$ & -0.3 & 32.7 & \\
\hline & 587.4674 & $\mathrm{C} 16: 2 / \mathrm{C} 18: 3 / \mathrm{OH}$ & 0.8 & 34.6 & MS2 \\
\hline & 589.4839 & $\mathrm{C} 16: 1 / \mathrm{C} 18: 3 / \mathrm{OH}$ & 2.2 & 10.3 & \\
\hline & 591.4989 & $\mathrm{C} 16: 0 / \mathrm{C} 18: 3 / \mathrm{OH}$ & 1.1 & 12.2 & \\
\hline & 613.4830 & C16:1/C20:5/OH & 0.6 & 46.2 & MS2 \\
\hline & 615.4990 & $\mathrm{C} 16: 0 / \mathrm{C} 20: 5 / \mathrm{OH}$ & 1.2 & 59.5 & MS2 \\
\hline & 639.4991 & $\mathrm{C} 18: 2 / \mathrm{C} 20: 5 / \mathrm{OH}$ & 1.2 & 3.7 & \\
\hline & 641.5144 & C18:1/C20:5/OH & 0.8 & 13.9 & MS2 \\
\hline & 661.4832 & $\mathrm{C} 20: 5 / \mathrm{C} 20: 5 / \mathrm{OH}$ & 0.9 & 103.9 & MS2 \\
\hline & 665.5152 & $\mathrm{C} 20: 4 / \mathrm{C} 20: 4 / \mathrm{OH}$ & 2.0 & 4.8 & MS2 \\
\hline
\end{tabular}


Table 1 continued

\begin{tabular}{llllcr}
\hline & $m / z$ & Assignment & Mass error $(\mathrm{ppm})$ & Relative intensity & Supporting information \\
\hline Non-fatty acids & 369.3512 & Cholesterol $-\mathrm{H}_{2} \mathrm{O}$ & -1.0 & 245.9 & \\
& 379.3355 & Ergosterol- $\mathrm{H}_{2} \mathrm{O}$ & -1.1 & 44.0 & \\
& 395.3667 & Stigmasterol- $\mathrm{H}_{2} \mathrm{O}$ & -1.4 & 76.2 & \\
& 411.3981 & Squalene & -0.9 & 23.0 & \\
& 431.3881 & $\alpha$-Tocopherol $($ vitamin E) & -0.6 & 15.4 & MS2 \\
& 871.5749 & Chlorophyll $a\left[-\mathrm{Mg}^{2+}+2 \mathrm{H}\right]$ & 2.0 & 173.2 & MS2 \\
\hline
\end{tabular}

All the compounds are detected as protonated; i.e., $[\mathrm{M}+\mathrm{H}]^{+}$

Relative intensity is in normalization to the most intense TAG ion $(\mathrm{m} / \mathrm{z} 851.7138)$ as 100

Structural assignments were confirmed with MS/MS (and MS3) for high abundance TAG and DAG (see Supplementary Table 1). Other TAG and DAG are best estimates

manual sample-loop injection with a syringe pump and an autosampler with LC pump (without LC column). With high solvent flow $\left(75 \mu \mathrm{L} \mathrm{min}{ }^{-1}\right)$ and low sample volume in the loop $(10 \mu \mathrm{L})$, only a few seconds were required to deliver the sample to the mass spectrometer and lasted about $20 \mathrm{~s}$ including tails. So, each analysis could take just $30 \mathrm{~s}$. Using the autosampler-LC pump set-up, it took another minute for the autosampler injection and instrument communication, and we were able to run a batch of samples injecting each sample every one and half minutes. To avoid contamination, we typically added a washing run between each sample but sample carry-over was negligible. High-throughput MS/MS analysis was tested by including data-dependent MS/MS for the $\mathrm{m} / \mathrm{z}$ range of TAG and DAG. This method automatically selected the most abundant three ions for subsequent MS/MS in each MS cycle with dynamic exclusion of previously selected ions. Typically, about forty MS/MS spectra could be obtained in $20 \mathrm{~s}$ of sample elution time, covering most of the abundant TAG and DAG species. For low abundance TAG and DAG ions and MS3 of a few TAG species, each independent run was made to perform MS/MS or MS3 of specific ions. Once all the MS/MS and MS3 information was acquired, it was not necessary to perform the same experiments for other samples that originated from the same algae species.

We performed two experiments to verify the quantification ability of our approach. First, we confirmed the linearity of ion signals in regards to sample concentration. At various algal oil concentrations (0.0005-0.002\%), we found a good linear relation between sample concentration and ion signals for each lipid species $\left(r^{2}>0.95\right.$; Supplementary Figure 2). We also performed absolute quantification of cholesterol using the standard-addition method. Different amounts of standard cholesterol (final concentration to $50-200 \mu \mathrm{M}$ ) were added to $0.01 \%$ algal oils. With $x$-axis extrapolation and considering the dilution factor, we determined the original cholesterol concentration as $100 \mathrm{mM}$, which is in good agreement with the result from a separate GC-MS experiment $(130 \mathrm{mM}$; see supplementary data).

Finally, to demonstrate the usefulness of the developed protocol for high-throughput process monitoring of algal oils, a set of algal oils was analyzed after the treatment with various MSN. Previous research found that nanopores in MSN materials have the capability of sequestering FFA [16]. Specifically, FFA were selectively sequestered over TAG, ergosterol, or squalene from a simulated solution by aminopropyl functional MSN. The analysis protocols adapted in the previous study require extensive procedures and analysis time, such as transesterification of TAG and silylation of sterols followed by GC-MS analysis. In addition, the analysis of TAG is through the indirect analysis of fatty acid components after the transesterification to fatty acid methyl esters, not the intact TAG species. The current protocol has several advantages over the conventional GC-MS method used previously. Most of all, it can directly analyze TAG and sterols without any chemical modifications, and with a much faster analysis time.

Table 2 shows the percentages of major algal oil components that remained after algal oil was treated with various MSN materials. Aminopropyl functionalized MSN with three different nanopore sizes $(3,5$, and $10 \mathrm{~nm})$ were tested along with 10-nm pore sized non-functionalized MSN, AP-MSN-3, AP-MSN-5, AP-MSN-10, and MSN-10, respectively. When both $10-\mathrm{nm}$ pore sized MSN (APMSN-10 and MSN-10) are compared, the aminopropyl surface functionalized MSN sequestered fewer overall lipids compared to non-functionalized MSN. Especially for chlorophyll $a$, only $15 \%$ is sequestered by AP-MSN-10 while $78 \%$ was sequestered by MSN-10. Regarding the pore size, sequestration was the greatest with the $3-\mathrm{nm}$ pore sized MSN in spite of aminopropyl surface functionalization, capturing $72 \%$ of the DAG, $25 \%$ of cholesterol, $42 \%$ of ergosterol and $82 \%$ of chlorophyll $a$. We 
Table 2 Semi-quantitative comparison of each algal lipid after various nanoparticle treatment

\begin{tabular}{|c|c|c|c|c|c|}
\hline & Control & AP-MSN-3 & AP-MSN-5 & AP-MSN-10 & MSN-10 \\
\hline TAG & $100(8.2)$ & $103(13.5)$ & $106(12.7)$ & $117(14.3)$ & 122 (11.9) \\
\hline DAG & $100(8.6)$ & $28(12.0)$ & $79(14.2)$ & $59(13.7)$ & $35(14.3)$ \\
\hline Cholesterol & $100(8.0)$ & $75(10.0)$ & $96(11.8)$ & $100(16.5)$ & 94 (11.7) \\
\hline Ergosterol & $100(3.3)$ & $58(14.5)$ & $88(14.7)$ & $85(15.6)$ & $74(13.5)$ \\
\hline Stigmasterol & $100(2.9)$ & $84(4.4)$ & $102(13.1)$ & $101(3.1)$ & $93(4.8)$ \\
\hline Squalene & $100(72.6)$ & $56(55.8)$ & 79 (35.6) & $70(43.1)$ & $74(33.4)$ \\
\hline$\alpha$-Tocopherol & $100(4.0)$ & 89 (13.2) & $94(12.9)$ & $106(11.7)$ & $111(22.5)$ \\
\hline Chlorophyll $a$ & $100(2.5)$ & $18(27.3)$ & $85(11.2)$ & $85(8.0)$ & $22(22.5)$ \\
\hline
\end{tabular}

TAG and DAG are the sums of ion intensities of all TAG and DAG compounds

Relative quantification was made in normalization to the corresponding ion signals in the control

Standard deviation is given in parenthesis

hypothesize that size effect is dominant for very small pore size. Namely, when the molecular size is slightly smaller than the pore size, it is difficult to escape once the molecules are trapped inside the pore network. This can explain why chlorophyll $a$ with the porphyrin ring size of $1.5 \mathrm{~nm}$ can be efficiently sequestered by AP-MSN-3. In contrast, with larger pore sizes, which allow for more freedom of movement of lipids within the pores, surface adsorption plays a major role for sequestration and aminopropyl functionalization effectively repels non-specific adsorption of most molecules while non-functionalized MSN sequester various classes of molecules non-selectively through non-specific interactions.

\section{Conclusion}

We demonstrated the ability of high resolution mass spectrometry combined with APCI to analyze a wide range of lipid compounds from crude algal oil in a highthroughput manner without further purification or separation, such as HPLC or SPE. Using this approach, a total of 32 TAG species were identified from algal crude oils based on accurate mass and further structural conformation was obtained for nineteen of them by MS/MS and MS3. Several other lipid species such as DAG, cholesterol, sterols, squalene and chlorophyll $a$ could be characterized at the same time in one and half minutes of total run time using an autosampler-LC.

This approach has pros and cons compared to other analytical approaches. Compared to GC-MS, it cannot analyze FFA but can analyze intact TAG species in molecular detail. Molecular speciation of TAG is inferior to HPLC-ESI-MS(/MS); however, it can effectively analyze non-polar or partially-polar compounds and provide much faster sample analysis. MALDI-MS allows a much higher throughput, but it suffers from significant ion suppression of TAG by chlorophyll and is inefficient for small lipid analysis. Encompassing this entire approach, it is very well suited for the high throughput monitoring of major lipids in algal crude oils. This assay successfully demonstrated semi-quantitative comparisons between algal oil components after treatment with various MSN materials. The algal crude oil used in the current study, however, is much less complex than raw microalgal extracts, lacking most of the polar compounds such as phospholipids. Hence, further study is necessary with microalgal extracts to make this approach more useful for the next generation of bio-fuel.

Acknowledgments We thank Adam Klein for his help in setting up the autosampler-LC configuration. This work is supported by U.S. Department of Energy, Office of Energy Efficiency and Renewable Energy (DE-FG26-0NT08854). The Ames Laboratory is operated by Iowa State University under DOE Contract DE-AC02-07CH11358.

\section{References}

1. Singh NK, Dhar DW (2011) Microalgae as second generation biofuel. Rev Agron Sustain Dev 31:605-629

2. Williams PJL, Laurens LML (2010) Microalgae as biodiesel \& biomass feedstocks: review \& analysis of the biochemistry, energetics \& economics. Energy Environ Sci 3:554-590

3. Gong Y, Jiang M (2011) Biodiesel production with microalgae as feedstock: from strains to biodiesel. Biotechnol Lett 33:12691284

4. Francisco EC, Neves DB, Jacob-Lopes E, Franco TT (2010) Microalgae as feedstock for biodiesel production: carbon dioxide sequestration, lipid production and biofuel quality. J Chem Technol Biotechnol 85:395-403

5. Krohn BJ, McNeff CV, Yan B, Nowlan D (2011) Production of algae-based biodiesel using the continuous catalytic Mcgyan (R) process. Bioresour Technol 102:94-100

6. Bigelow NW, Hardin WR, Barker JP, Ryken SA, MacRae AC, Cattolico RA (2011) A comprehensive GC-MS sub-microscale assay for fatty acids and its applications. J Am Oil Chem Soc 88:1329-1338 
7. Forjan E, Garbayo I, Henriques M, Rocha J, Vega JM, Vilchez C (2011) UV-A mediated modulation of photosynthetic efficiency, xanthophyll cycle and fatty acid production of nannochloropsis. Mar Biotechnol 13:366-375

8. Yu ET, Zendejas FJ, Lane PD, Gaucher S, Simmons BA, Lane TW (2009) Triacylglycerol accumulation and profiling in the model diatoms Thalassiosira pseudonana and Phaeodactylum tricornutum (Bacillariophyceae) during starvation. J Appl Phycol 21:669-681

9. MacDougall KM, McNichol J, McGinn PJ, O'Leary SJB, Melanson JE (2011) Triacylglycerol profiling of microalgae strains for biofuel feedstock by liquid chromatography-high-resolution mass spectrometry. Anal Bioanal Chem 401:2609-2616

10. Danielewicz MA, Anderson LA, Franz AK (2011) Triacylglycerol profiling of marine microalgae by mass spectrometry. J Lipid Res 52:2101-2108

11. He H, Rodgers RP, Marshall AG, Hsu CS (2011) Algae polar lipids characterized by online liquid chromatography coupled with hybrid linear quadrupole ion trap/Fourier transform ion cyclotron resonance mass spectrometry. Energy Fuels 25:47704775

12. Rezanka T, Lukavsky J, Nedbalova L, Sigler K (2011) Effect of nitrogen and phosphorus starvation on the polyunsaturated triacylglycerol composition, including positional isomer distribution, in the alga Trachydiscus minutus. Phytochemistry 72: 2342-2351

13. Chen H-T, Huh S, Wiench JW, Pruski M, Lin VSY (2005) Dialkylaminopyridine-functionalized mesoporous silica nanosphere as an efficient and highly stable heterogeneous nucleophilic catalyst. J Am Chem Soc 127:13305-13311

14. Zhao Y, Trewyn BG, Slowing II, Lin VSY (2009) Mesoporous silica nanoparticle-based double drug delivery system for glucose-responsive controlled release of insulin and cyclic AMP. J Am Chem Soc 131:8398-8400

15. Giri S, Trewyn BG, Stellmaker MP, Lin VSY (2005) Stimuliresponsive controlled-release delivery system based on mesoporous silica nanorods capped with magnetic nanoparticles. Angew Chem Int Ed 44:5038-5044

16. Valenstein JS, Kandel K, Melcher F, Slowing II, Lin VSY, Trewyn BG (2012) Functional mesoporous silica nanoparticles for the selective sequestration of free fatty acids from microalgal oil. Acs Appl Mat Interf 4:1003-1009

17. Byrdwell WC, Neff WE (2002) Dual parallel electrospray ionization and atmospheric pressure chemical ionization mass spectrometry (MS), MS/MS and MS/MS/MS for the analysis of triacylglycerols and triacylglycerol oxidation products. Rapid Commun Mass Spectrom 16:300-319

18. Byrdwell WC (2001) Atmospheric pressure chemical ionization mass spectrometry for analysis of lipids. Lipids 36:327-346

19. Khozin-Goldberg I, Boussiba S (2011) Concerns over the reporting of inconsistent data on fatty acid composition for microalgae of the genus Nannochloropsis (Eustigmatophyceae). J Appl Phycol 23:933-934 


\title{
APPENDIX 4
}

\section{THESIS CHAPTER \\ CHAPTER 4. ORGANIC FUNCTIONALIZED MESOPOROUS SILICA NANOPARTICLES WITH LARGE PORE DIAMETERS AS EFFICIENT HETEROGENEOUS CATALYSTS}

Wei Huang, Chih-Hsiang Tsai, Takeshi Kobayashi, Marek Pruski, Hung-Ting Chen, * Brain G. Trewyn, ${ }^{*}$ and Victor S.-Y. Lin

\begin{abstract}
A series of organic-functionalized large-pore mesoporous silica nanoparticles (LPMSNs) were synthesized via the co-condensation method using a Pluronic 104 triblock copolymer as the pore forming surfactant in acidic conditions. We systematically investigated synthetic parameters, such as the pre-hydrolysis time for tetraethyl orthosilicate and aging temperature, to obtain rice-shaped individual functionalized LPMSNs with large pore diameters ranging from 5 $\mathrm{nm}$ to $10 \mathrm{~nm}$. We discovered that the pre-hydrolysis time was crucial to control particle shape and morphology. Whereas the temperature of hydrothermal process did not influence morphology but affected the chemical accessibility of surface functional groups. The thiolfunctionalized LPMSN was oxidized by hydrogen peroxide to convert thiols into sulfonic acids. The catalytic activity of the resulting acid-functionalized LPMSN was examined and it demonstrated higher esterification activity than acid-functionalized SBA-15. This result demonstrated the importance in the size control of mesoporous silica supports in terms of the catalytic reactivity.
\end{abstract}

\section{Introduction}

Since the discovery of M41S family, ${ }^{1,2}$ mesoporous silica materials have attracted extensive interest due to their important structural properties such as large surface area, tunable pore diameter, and feasible surface modification. It was soon after the discovery of mesoporous silicas that the interest in this field has focused on pore expansion and morphology control, which are two extremely important physical parameters for industrial applications. ${ }^{3-10}$ Many research groups, including our own, have successfully controlled particle size and morphology of MCM-41 type materials to produce mono-dispersed mesoporous silica nanoparticles (MSNs) with pore diameter in the range of $2 \sim 3 \mathrm{~nm}$ and applied as a carrier in controlled-release drug delivery $^{11-16}$ and as supports in heterogeneous catalysis. ${ }^{17-21}$ To further expand the application area, much effort has been devoted to enlarging the pore size of this material. ${ }^{22-24}$ The two strategies for pore expansion in MCM-41 that are most commonly utilized are long alkyl chain surfactants or a hydrophobic organic additive swelling agent used in addition to surfactant pore formers. However both these strategies also have several disadvantages. For example, the room for pore size enlargement is small when applying long alkyl chain surfactants such as hexadecyltrimethylammonium salts. ${ }^{1}$ Also, surfactants with longer carbon chains $\left(>\mathrm{C}_{22}\right)$ need to be synthesized from expensive precursors. Adding a swelling agent such as 1,3,5trimethylbenzene (TMB) significantly expands the mesopore diameter up to $10 \mathrm{~nm} ;{ }^{1,25}$ however, the long-range pore structural ordering is lost with pores larger than $5 \mathrm{~nm} .^{26}$ In addition, the particle size and shape are not well-controlled as the pore size increases.

SBA-15 is a notable mesoporous silica material with a much large pore diameter, was 
discovered by Stucky and co-workers, who reported a synthetic procedure using an amphiphilic triblock copolymer Pluronic 123, poly(ethyleneoxide) $20^{2}$-block-poly(propyleneoxide) 70 -blockpoly(ethyleneoxide) $)_{20}$, as the structure-directing agent in acidic condition. ${ }^{27}$ The SBA-15 materials exhibited a 2D hexagonal arrangement of highly ordered cylindrical mesopores with tunable pore size varied from $5 \mathrm{~nm}$ to $30 \mathrm{~nm}$. Nevertheless, given that the wheat-like macrostructure was observed in the conventional SBA-15, the morphology control of the SBA15 type materials has been extensively studied. Many synthetic methods, providing individual isolated particles, were reported including adding inorganic salts (such as $\mathrm{KCl}^{28}{ }^{28} \mathrm{NaCl}^{29}$ ), using co-solvent (like $\mathrm{EtOH}^{30}$ ), and introducing co-surfactant (for example cationic surfactant together with nonionic surfactant ${ }^{31}$ ) in the reaction mixture. Despite some success that has been made in the synthesis of diverse shaped SBA-15 material, ${ }^{32-36}$ control of SBA-15 particle size with channel depth of sub-micrometer is still a challenge. Moreover, most morphology studies were focused on pure silica materials; little attention was paid on functionalized ones. Although one can argued that surface functionalization of these materials could be achieved through a postgrafting technique, loading of surface functional groups obtained by this approach was restricted to the number of available silanols, which were especially limited for calcined samples. Thus, it is of great interest to develop a new method to fabricate mesoporous silica nanoparticles with regular particle size, large pore diameter, and also containing highly loaded surface organic functionality.

Herein, we report a synthetic procedure combining a co-condensation method with small particle preparation to provide organic functionalized large-pore mesoporous silica nanoparticles (LPMSNs). These LPMSNs are well-isolated particles of regular, uniform sizes (500 800 nm) by systematically refining two synthetic steps: hydrothermal temperature and the sequence of reagent addition. Five organic silanes, including 3-mercaptopropyl trimethoxysilane (MPTMS), 3-cyanopropyl trimethoxysilane (CPTMS), 3-aminopropyl trimethoxysilane (APTMS), triethoxysilylbutyraldehyde (ADTES), and 3-cyclopentadienylpropyltriethoxysilane (CPDTES), were specifically used in this study because of their potential as linkers for further conjugation with reactive homogeneous catalysts or biological macromolecules for immobilization.

\section{Experimental Section}

Chemicals. All chemicals were used as received without further purification. Tetramethyl orthosilicate (TMOS) and palmitic acid were purchased from Sigma-Aldrich. All organic silanes, including 3-mercaptopropyltrimethoxysilane (MPTMS), 3aminopropyltrimethoxysiliane (APTMS), 3-cyanopropyltrimethoxysilane (CPTMS), triethoxysilylbutyraldehyde (ADTES), and 3-cyclopentadienylpropyltriethoxysilane (CPDTES), were purchased from Gelest. The Pluronic 104 triblock copolymer, $\mathrm{PEO}_{27} \mathrm{PPO}_{61} \mathrm{PEO}_{27}$, was obtained from BASF.

Synthesis of thiol functionalized large-pore mesoporous silica nanoparticle material with different MPTMS addition procedure: A P104 triblock copolymer ( $7.0 \mathrm{~g})$ was dissolved in $1.6 \mathrm{M} \mathrm{HCl}_{(a q)}$ solution $(273.4 \mathrm{~g})$ and stirred vigorously at $56{ }^{\circ} \mathrm{C}$ for $30 \mathrm{~min}$. To the clear solution, TMOS (10.6 g, $69.9 \mathrm{mmol})$ was added quickly and pre-hydrolyzed for $x$ min, where $x=$ 0,15 or 30 , followed by a quick injection of MPTMS (1.6 g, $8.4 \mathrm{mmol}, 12 \mathrm{~mol} \%$ of total TMOS). The reaction mixture was kept at $56{ }^{\circ} \mathrm{C}$ for $24 \mathrm{~h}$ and transferred to a Teflon-lined highpressure autoclave for aging hydrothermally at $100{ }^{\circ} \mathrm{C}$. The as-synthesized material was isolated by filtration, washed with copious amounts of water and methanol, and dried under vacuum.

Synthesis of thiol functionalized large-pore mesoporous silica nanoparticle material 
with varying aging temperatures: The synthetic procedure was similar to the aforementioned method, except that MPTMS was added 15 min after injection of TMOS, and the sample was hydrothermally treated at either 100 or $150{ }^{\circ} \mathrm{C}$.

General synthetic procedure of preparing organic functionalized large-pore mesoporous silica materials: The functionalized mesoporous silica material was synthesized in the optimized procedure as described before by pre-hydrolyzed TMOS for $30 \mathrm{~min}$, followed by the addition of organic silane, and the material was aged at $100{ }^{\circ} \mathrm{C}$ for $24 \mathrm{~h}$. The introduced amount of organic silane kept at $12 \mathrm{~mol} \%$ with respect to the TMOS, for all silanes, except for APTMS, which was used at $5 \mathrm{~mol} \%$.

General extraction procedure for surfactant removal: The aforementioned material $(1.0 \mathrm{~g})$ was refluxed in ethanol $(250 \mathrm{ml})$ for $24 \mathrm{~h}$ for removal of the P104 surfactant. The material was filtered, washed with ethanol, and dried under vacuum to yield surfactant-extracted mesoporous silica sample.

Material Characterization: Powder X-ray diffraction (PXRD) was performed on a 2Theta type $\mathrm{X}$-ray diffractometer by Rigaku Corporation using $\mathrm{Cu}-\mathrm{K} \alpha$ radiation. The nitrogen gas sorption analysis was measured on a Micromeritics ASAP 2020 analyzer. A thermogravimetric analyzer 2950 manufactured by TA Instruments was used for thermogravimetric analysis (TGA) measurement of silica samples. The samples were heated from room temperature to $800{ }^{\circ} \mathrm{C}$ at ramping rate of $2{ }^{\circ} \mathrm{C} / \mathrm{min}$ in air. Scanning electron microscopy (SEM) images were taken on a Quanta 250 FEG Scanning Electron Microscope made by the FEI Company. Transmission electron microscopy (TEM) micrographs were carried out on a FEI Tecnai G ${ }^{2}$ F20 STEM working at $200 \mathrm{kV}$. A acid-base titration was applied to determine the quantity of sulfonic acid groups in the mesoporous silica material according to the reported method. ${ }^{37}$ Solid-state ${ }^{13} \mathrm{C}$ NMR spectra were taken under the following conditions: $14.1 \mathrm{~T}$ Varian NMR system with a 3.2 $\mathrm{mm}$ probe operated at $599.6 \mathrm{MHz}$ for ${ }^{1} \mathrm{H}$ and $150.8 \mathrm{MHz}$ for ${ }^{13} \mathrm{C}$, respectively. MAS rate $\mathrm{n}_{\mathrm{R}}=$ $20 \mathrm{kHz}, \mathrm{n}_{\mathrm{RF}}^{\mathrm{H}}=80 \mathrm{kHz}$ during short pulse, $\mathrm{CP}$ (tangent) and SPINAL64 ${ }^{1} \mathrm{H}$ decoupling, $\mathrm{n}_{\mathrm{RF}}{ }^{\mathrm{C}}=$ $60 \mathrm{KHz}, \tau_{\mathrm{CP}}=1.5 \mathrm{~ms}, \tau_{\mathrm{RD}}=1.5 \mathrm{~s}$ and $\mathrm{NS}=20000$. The spectra are normalized for constant height for visual clarity. ${ }^{29} \mathrm{Si}$ DPMAS spectra were obtained under the following conditions: 9.4 T Chemagnetics CMX Infinity system with a $5 \mathrm{~mm}$ probe operated at $400.0 \mathrm{MHz}$ for ${ }^{1} \mathrm{H}$ and $79.5 \mathrm{MHz}$ for ${ }^{29} \mathrm{Si}$, respectively. MAS rate $\mathrm{n}_{\mathrm{R}}=10 \mathrm{kHz}, \mathrm{n}_{\mathrm{RF}}{ }^{\mathrm{Si}}=50 \mathrm{KHz}, \mathrm{n}_{\mathrm{RF}}{ }^{\mathrm{H}}=40 \mathrm{KHz}$ during short pulse and TPPM decoupling, $\mathrm{N}_{\mathrm{CPMG}}=10, \tau_{\mathrm{CPMG}}=10 \mathrm{~ms}, \tau_{\mathrm{RD}}=300 \mathrm{~s}$ and $\mathrm{NS}=296$. The spectra are normalized for constant height for visual clarity.

Synthesis of sulfonic acid-functionalized large-pore mesoporous silica nanoparticles by oxidation: Mercaptopropyl-functionalized material $(300.0 \mathrm{mg})$ was treated with $30 \%$ $\mathrm{H}_{2} \mathrm{O}_{2(a q)}$ solution for $24 \mathrm{~h}$ for oxidation under Ar. The material was filtered, washed with water and ethanol, and re-suspended in $30 \mathrm{ml}$ of $1 \mathrm{M} \mathrm{H}_{2} \mathrm{SO}_{4(a q)}$ for protonation to yield an acid functionalized silica material.

Kinetic study of esterification reaction catalyzed by sulfonic acid-functionalized mesoporous silica material: A mixture of palmitic acid $(245.5 \mathrm{mg})$, methanol $(120 \mu \mathrm{l})$, and catalyst ( $1 \mathrm{~mol} \%$ of sulfonic acid group with respect to palmitic acid) in $5 \mathrm{ml}$ of heptanes was stirred continuously at $60{ }^{\circ} \mathrm{C}$. A small portion of reaction mixture was withdrawn at desired times, and the conversion of palmitic acid to methyl palmitate was determined by ${ }^{1} \mathrm{H}-\mathrm{NMR}$ using the integrated area of methyl protons $(\mathrm{s}, \delta=3.66)$ and $\alpha$-methylene protons $(\mathrm{t}, \delta=2.28-2.37)$.

\section{Results and Discussion}

Several studies, include our own, reported that introduction of organic silanes in materials 
influence not only the structure ordering but also the morphology of the final mesoporous silica materials. To avoid the perturbation of final particle morphology, we have systematically investigated synthetic parameters to find a way that to incorporate organic functionality and also maintain individually isolated particles. The MPTMS was used as the model silane for optimization of functionalized LPMSN synthesis due to its potential precursor as an acid catalyst.

\section{Prehydrolysis of TMOS}

First, we studied the influence of TMOS prehydrolysis time in terms of final particle morphology. The TMOS was introduced to a surfactant solution and allowed to pre-hydrolyze for $x$ min before injection of the functional silane, MPTMS, where $x=0,15$, and 30, respectively. The resulting MP-LPMSN was denoted as MP-LPMSN- $x$ for simplification. The SEM micrographs of MP-LPMSNs showed that the resulting material had very different morphology depend on the addition time of MPTMS as depicted in Figure 4-1. As shown in Figure 4-1(a), the unfunctionalized LPMSN synthesized based on the literature procedure exhibited mono-dispersed individual hexagonal-shaped particles. However, introducing TMOS and MPTMS simultaneously to the system altered these individual hexagonal particles to aggregations of irregular elongated curved tubes. If the TMOS was allowed to be prehydrolyzed for $15 \mathrm{~min}$ before addition of MPTMS, the aggregation of primary MP-LPMSN particles diminished, which resulted in poorly separated oval-shaped particle as shown in SEM image of MP-LPMSN-15. Further extension of the prehydrolysis time to 30 min prevented the fusion of particles. The SEM images of MP-LPMSN-30 in Figure 4-1(d) exhibited individual hexagonal particles similar to unfunctionalized LPMSN. In addition, the prehydrolysis of TMOS for longer time also increased the surface area and narrowed pore size distribution as shown in Table 4-S1. The result indicated that that the pre-hydrolysis of TMOS was critical to control particle morphology of the functionalized LPMSN materials. By adding MPTMS at 30 min after injection of TMOS, the uniform well-isolated MP-LPMSNs could be obtained under the reaction conditions. 

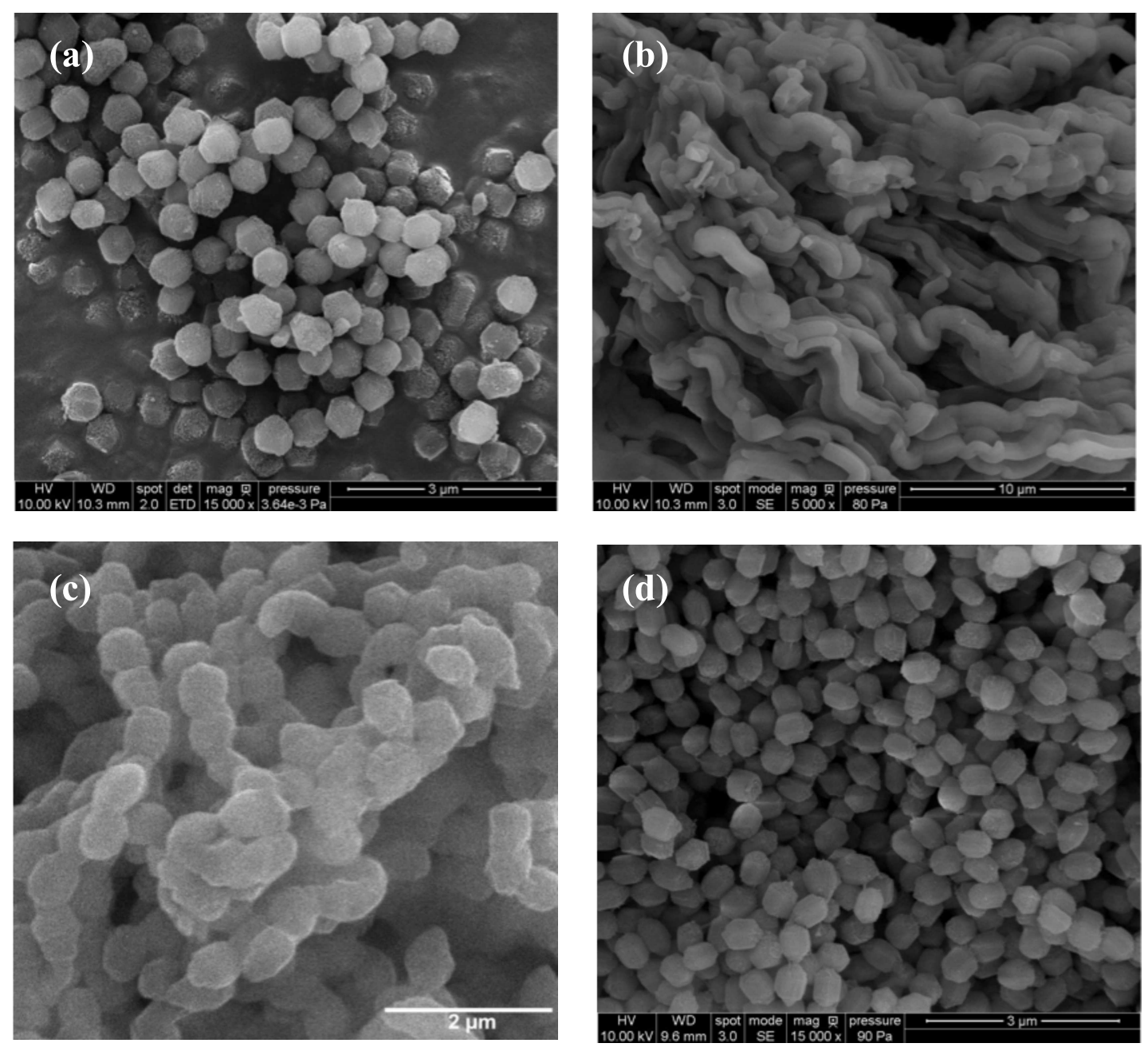

Figure 4-1. SEM images of (a) unfunctionalized LPMSN (scale bar $=3 \mu \mathrm{m}$ ), (b) MPLPMSN-0 (scale bar $=10 \mu \mathrm{m})$, $(\mathrm{c})$ MP-LPMSN-15 $($ scale bar $=2 \mu \mathrm{m})$, and $(\mathrm{d}) \mathrm{MP}-$ LPMSN-30 (scale bar $=3 \mu \mathrm{m})$.

\section{Aging temperature}

To investigate how the aging temperature affected the resulting MP-LPMSN material, the MP-LPMSNs were synthesized based on an aforementioned procedure and hydrothermally aged for $24 \mathrm{~h}$ at two different temperatures: 100 and $150{ }^{\circ} \mathrm{C}$. The resulting materials were labeled MP-LPMSN- $y$, where $y=100{ }^{\circ} \mathrm{C}$ or $150{ }^{\circ} \mathrm{C}$ depending on the aging temperature. The textural properties of the two MP-LPMSNs measured by nitrogen sorption analysis were summarized in Table 4-S2. The mean pore diameter of MP-LPMSN-150C exhibited a larger pore diameter of $6.1 \mathrm{~nm}$, calculated by BJH method, than MP-LPMSN-100C with a pore diameter of $4.6 \mathrm{~nm}$. The specific surface area of MP-LPMSN-150C $\left(576 \mathrm{~m}^{2} / \mathrm{g}\right)$, measured by the BET method, was less than that of MP-LPMSN-100C $\left(875 \mathrm{~m}^{2} / \mathrm{g}\right)$. This means that increasing aging temperature enlarged the pore diameter but decreased the surface area of the resulting materials. The ${ }^{29} \mathrm{Si}$ solid-state NMR studies were used as tools to investigate the nature of the incorporation of organic thiol functionality quantitatively and qualitatively in the mesoporous silica framework. As shown in the Figure 4-2, the existence of $\mathrm{T}$ sites in the ${ }^{29} \mathrm{Si}$ solid-state DPMAS spectra of MP-LPMSNs demonstrated that the mercaptopropyl group was covalently bonded on the silica 
surface via co-condensation. The amount of surface mercaptopropyl group was estimated by ${ }^{29} \mathrm{Si}$ DPMAS to be $1.2 \mathrm{mmol} / \mathrm{g}$ and $1.1 \mathrm{mmol} / \mathrm{g}$ for MP-LPMSN-100C and MP-LPMSN-150C, respectively. This result implies that the hydrothermal aging temperature had no effect on the quantity of incorporated organic functional group. The TGA analyses were also used to further confirm the inclusion of organic thiol group in the MP-LPMSN materials as depicted in Figure 4-3. The weight loss of MP-LPMSNs before $150{ }^{\circ} \mathrm{C}$ was assigned to the adsorbed solvent on the silica surface as shown in Figure 4-3 (a) and (b). A sharp drop in weight centered at around 300 ${ }^{\circ} \mathrm{C}$ indicated that the decomposition of mercaptopropyl groups in the two materials was close to the temperature found in the mercaptopropyl functionalized SBA-15.

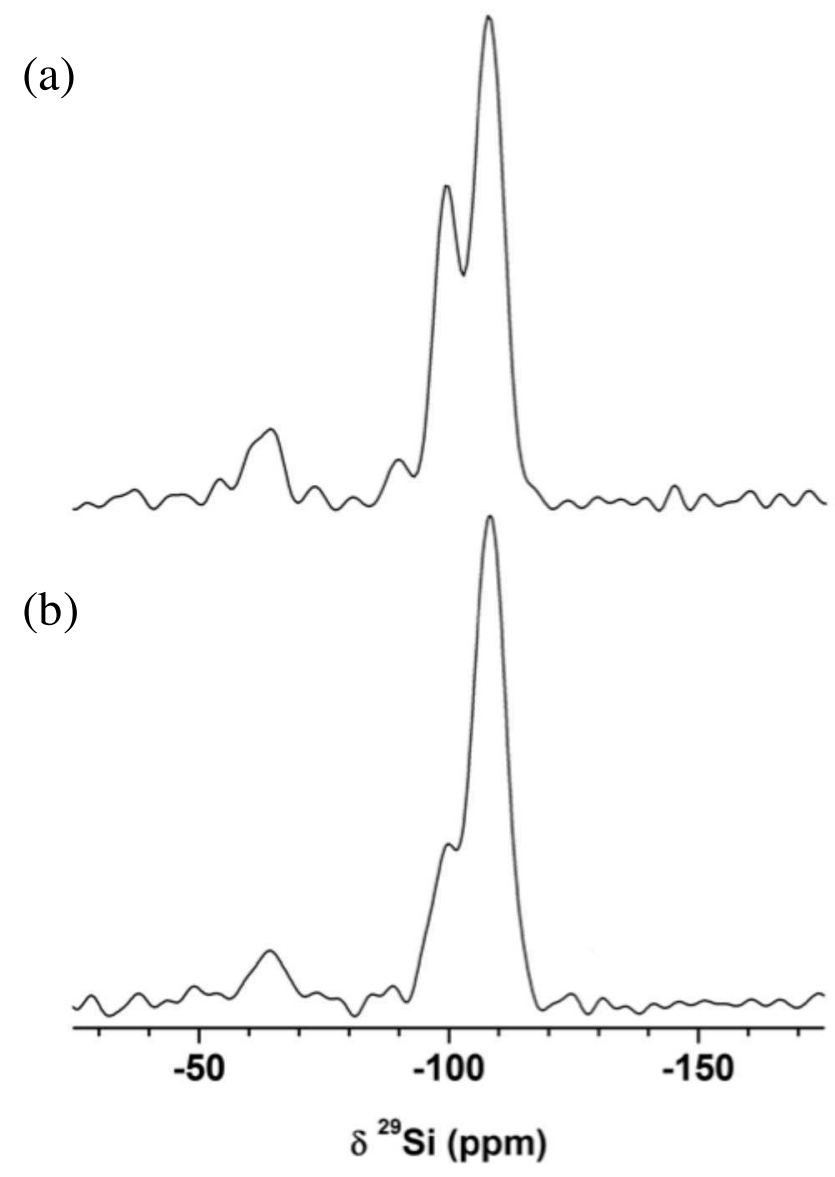

Figure 4-2. ${ }^{29} \mathrm{Si}$ DPMAS spectra of MP-LPMSN-100C (a) and MP-LPMSN-150C (b). 

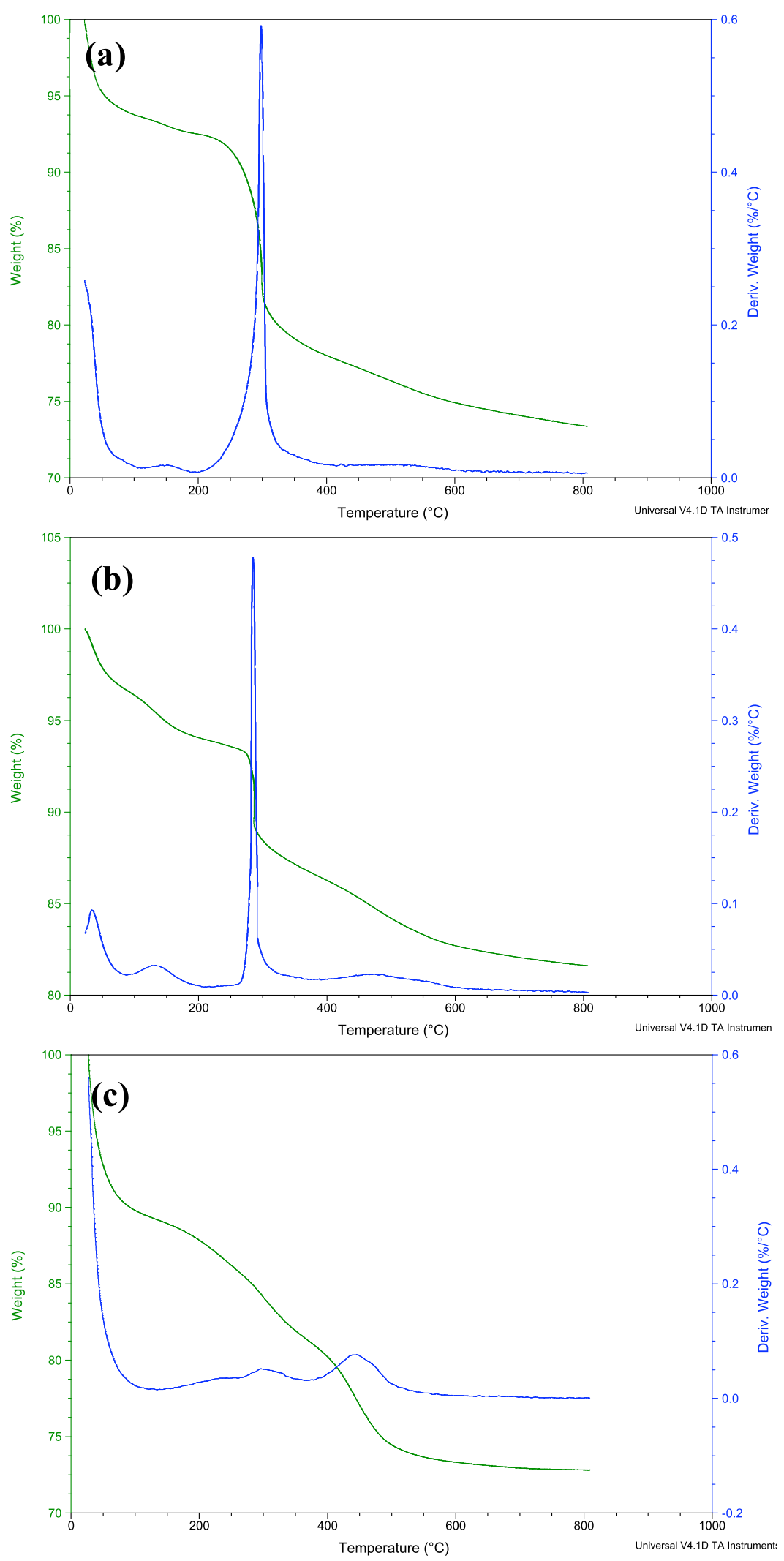

Final Progress Report 


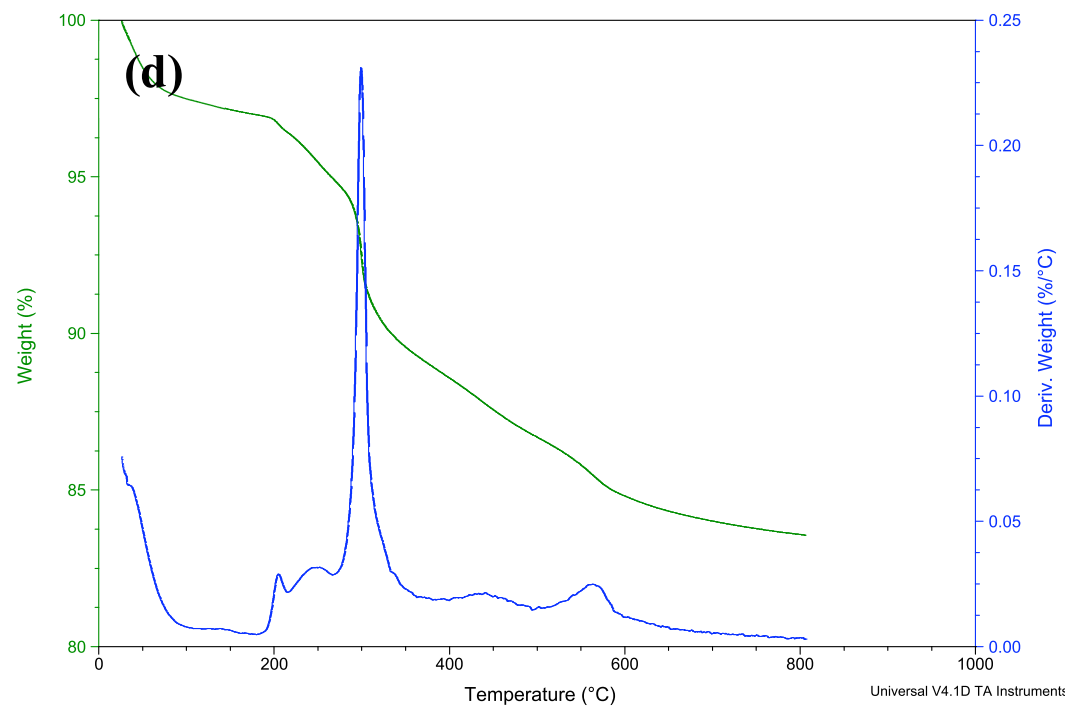

Figure 4-3. TGA analyses of (a) MP-LPMSN-100C, (b) MP-LPMSN-150C, (c) SA-LPMSN100C, and (d) SA-LPMSN-150C. The mass changes of MP-LPMSN samples were monitored from room temperature to $800{ }^{\circ} \mathrm{C}$ at a ramping rate of $2{ }^{\circ} \mathrm{C} / \mathrm{min}$ in air.

To further study the chemical accessibility of surface functional groups, the MP-LPMSN$100 \mathrm{C}$ and MP-LPMSN-150C were subjected to post-synthesis oxidation in $30 w t \% \mathrm{H}_{2} \mathrm{O}_{2(a q)}$ solution for $24 \mathrm{~h}$ to convert thiols into sulfonic acids, where the resulting materials were labeled as SA-LPMSN-100C and SA-LPMSN-150C, respectively. TGA measurement was performed on the sulfonic acid functionalized LPMSNs to qualitatively examine the conversion of sulfonic acid through oxidation as shown in Figure 4-3 (c) and (d). For SA-LPMSN-100C, a weight loss related to the decomposition of the thiol group was considerably decreased, but a dramatic weight change occurred when the temperature reached around $450{ }^{\circ} \mathrm{C}$ indicating the presence of sulfonic acid groups resulted from the oxidation of thiol functionalities. However, there was no observation of weight loss at $450{ }^{\circ} \mathrm{C}$, but at $300^{\circ} \mathrm{C}$, in the SA-LPMSN-150C that suggested that surface thiol groups remained intact after oxidation. The amount of sulfonic acid groups in both SA-LPMSNS were determined by acid-base titration based on a known procedure. ${ }^{37}$ Typically, the acid sample is treated with excess amount of sodium salt, where the chemically accessible acidic protons in the material could be ion-exchanged by sodium cations. The solid material is filtered and the filtrate is titrated with $0.01 \mathrm{M} \mathrm{NaOH}_{(a q)}$. Surprisingly, the sulfonic acid amount of SA-LPMSN-100C $(475 \mu \mathrm{mol} / \mathrm{g})$, calculated from this method, was found to be five times greater than that of SA-LPMSN-150C $(91 \mu \mathrm{mol} / \mathrm{g})$ as depicted in Table 4-S2. In other words, $41 \%$ of the thiol groups on MP-LPMSN-100C, but only $8 \%$ on MP-LPMSN-150C, were accessible to the hydrogen peroxide and successfully oxidized. This result could be attributed to the reorganization of silica framework during process of hydrothermal treatment. At higher aging temperature the mesoporous silica structure rearranged in a way that most of the thiol groups were embedded deeply inside the matrix, which became inaccessible and remained intact even in high concentration of the $\mathrm{H}_{2} \mathrm{O}_{2}$ solution. Aging functionalized LPMSNs at high temperature resulted in a significant reduction in the accessible surface functional groups although it offered larger pore size and similar surface concentration of organic groups.

\section{Synthesis of organic functionalized large-pore mesoporous silica nanoparticles:}

A series of organic functionalized LPMSNs were prepared from the reaction mixture containing organosilane/TMOS with molar ratio of $12 \%$, except for APTMS (5\%), by using a 
TMOS prehydrolysis step of $30 \mathrm{~min}$ and aging at $100{ }^{\circ} \mathrm{C}$ for $24 \mathrm{~h}$. During this investigation, we discovered that the basic APTMS could influence the $\mathrm{pH}$ value of the reaction mixture dramatically changing the porous structure of the final LPMSNs. Therefore, the initial amount of APTMS added was reduced from $12 \mathrm{~mol} \%$ to $5 \mathrm{~mol} \%$ with respect to TMOS. The as-made sample was extracted in refluxed ethanol to remove surfactant rather than calcination to preserve the organic functionality at low temperature. The SEM micrographs of all organic functionalized LPMSNs are displayed in Figure 4-4. All of LPMSN materials prepared under optimized synthetic conditions exhibited well-separated rice-shaped particles with particle size of about 700 $\times 380 \mathrm{~nm}$. The small-angle XRD patterns of CP-, AP-, and AD-LPMSNs, showed three intense diffraction peaks indexed as (100) (110) (200), these are associated with 2-D hexagonal symmetry as depicted in Figure 4-5. The MP- and CPD-MSN display only one (100) peak, indicative of less ordered structure when 3-mercaptopropyl and 3-cyclopentadienylpropyl groups were incorporated into the silica framework. 

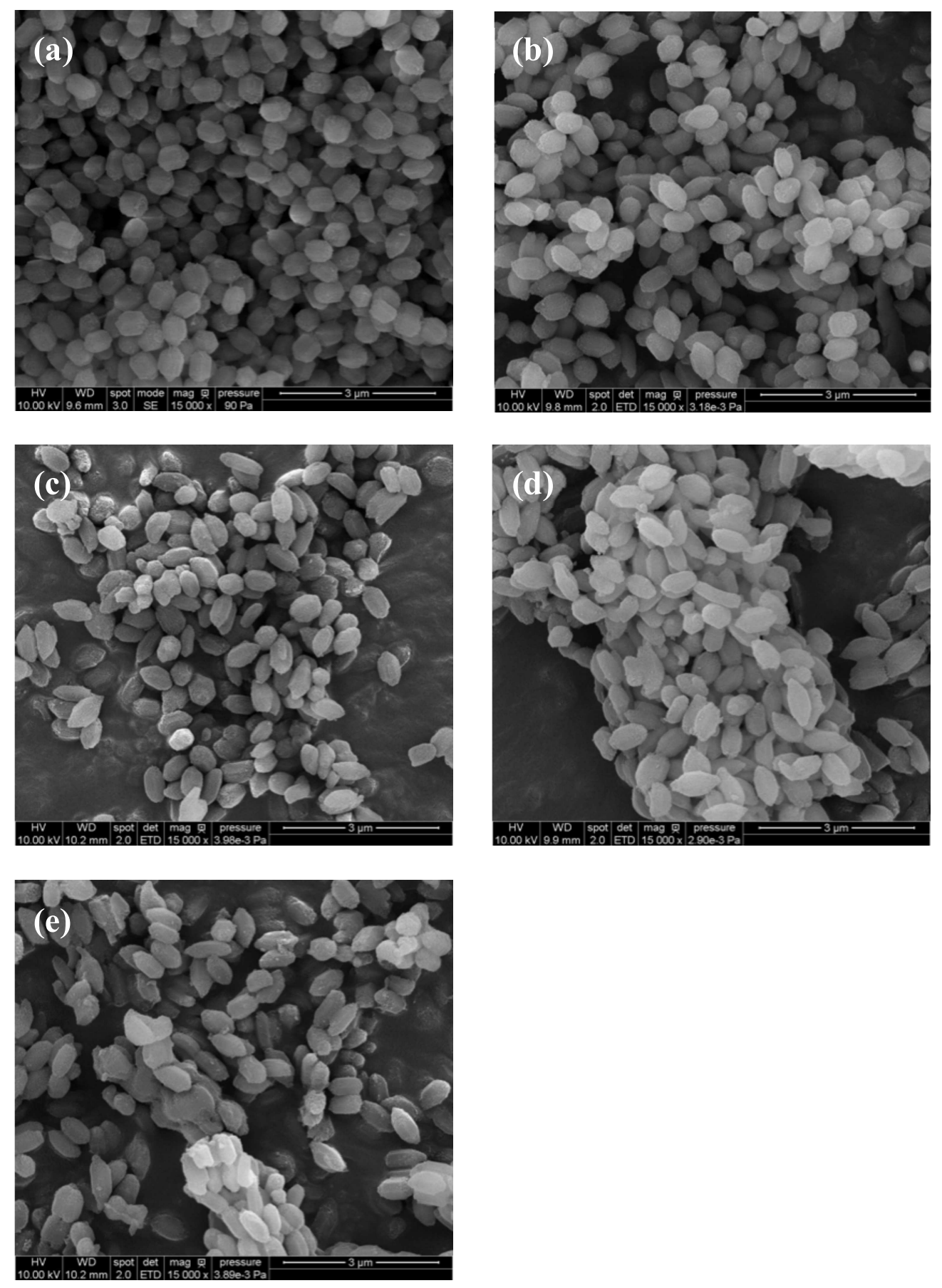

Figure 4-4. SEM images of MP-LPMSN (a), CP-LPMSN (b), AP-LPMSN (c), ADLPMSN (d), and CPD-LPMSN (e). All scale bar $=3.0 \mu \mathrm{m}$. 


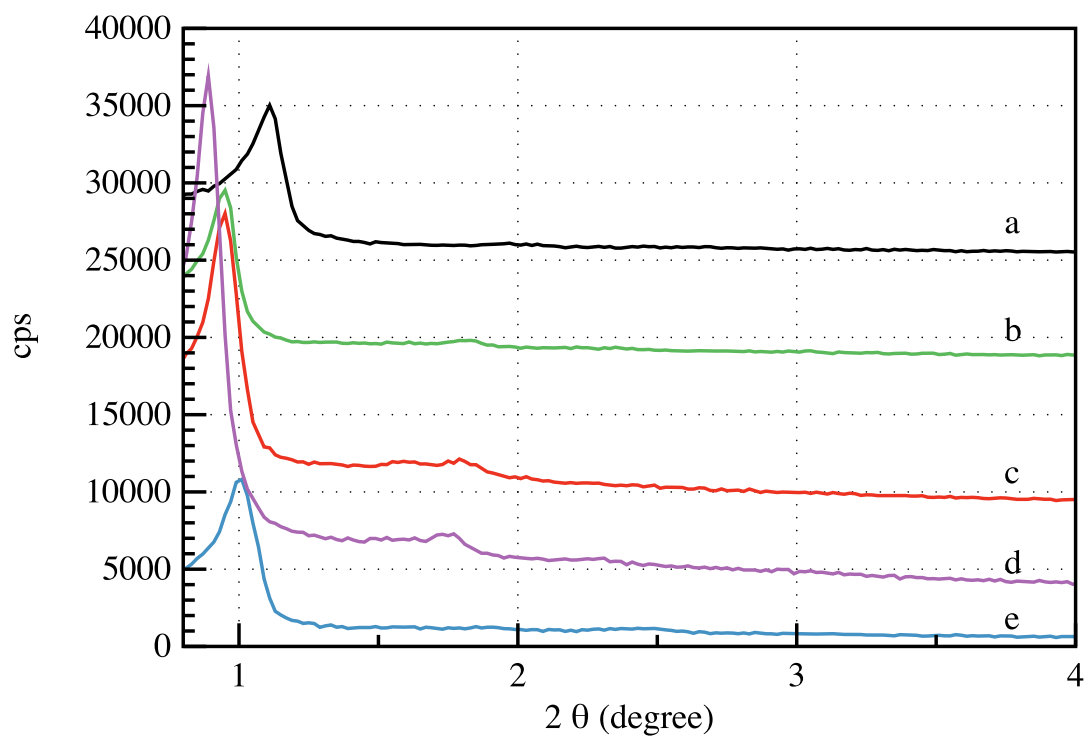

Figure 4-5. Power XRD patterns of MP-LPMSN (a), CP-LPMSN (b), AP-LPMSN (c), ADLPMSN (d), and CPD-LPMSN (e).

The nitrogen isotherms and pore size distribution of organic functionalized LPMSNs obtained by nitrogen sorption analysis were presented in Figure 4-S1. The type IV isotherms with $\mathrm{H}_{1}$-type hysteresis were observed in all functionalized LPMSN materials, indicative of hexagonally ordered, cylindrical-channeled mesoporous material. That was confirmed by powder XRD patterns mentioned in Figure 4-5. The LPMSNs showed a very sharp pore size distribution with pore diameters ranging from $5.3 \mathrm{~nm}$ to $7.4 \mathrm{~nm}$ depending on the types of organic functionality.

Carbon-13 solid-state CP-MAS NMR was employed to demonstrate the presence of organic functionalities in the mesopores and confirm the integrity of the structures as depicted in Figure 4-6. Each resonance peak was assigned based on the observed chemical shifts. The additional peak observed at around $60 \mathrm{ppm}$ most likely represents ethanol adsorbed on the surface during the extraction. The peaks associated with surfactant P104, shown at 18, 70, and 76 ppm, were also detected in the ${ }^{13} \mathrm{C}$ CPMAS NMR spectra of all functionalized LPMSNs, indicating some surfactant remained in the mesopores after ethanol extraction. As the case of SBA-15, it was reported that ethanol extraction did not completely remove surfactant, leaving about $6 \mathrm{wt} \%$ of total sample inside the mesopores. ${ }^{38}$ The functional LPMSN materials washed with additional hydrochloric acid in the ethanol or the extended time did not improve the extraction efficiency. Even so, the remained surfactant in the mesopores had little influence on the catalytic activity described here. The amount of organic functionality in the porous materials was estimated by ${ }^{29} \mathrm{Si}$ solid-state DPMAS spectra, giving similar loadings on the silica surface $(1.0 \sim 1.3 \mathrm{mmol} / \mathrm{g})$ except for the AP group $(0.7 \mathrm{mmol} / \mathrm{g})$. Elemental analysis was also performed on MP-, CP-, and AP-LPMSN samples to confirm the loading of functional groups. The CPD- and AD-LPMSNs did not take into account since these two samples contain only C, $\mathrm{H}$, and $\mathrm{O}$ atoms, which gave unreliable data due to adsorbed solvents or remained surfactants. The quantities of functional groups in the MP-, CP-, and AP-LPMSNs determined by elemental analysis agreed very well with number obtained by solid-state NMR studies as shown in Table 43.

Table 4-3. Structural properties of organic functionalized LPMSNs. 


\begin{tabular}{|c|c|c|c|c|c|}
\hline Material & $d_{100}(\mathrm{~nm})^{a}$ & $S_{B E T}\left(\mathrm{~m}^{2} \mathrm{~g}^{-1}\right)^{b}$ & $V_{p}\left(\mathrm{~cm}^{3} \mathrm{~g}^{-1}\right)^{b}$ & $\begin{array}{l}\mathrm{W}_{B J H} \\
(\mathbf{n m})^{b}\end{array}$ & $\begin{array}{l}\text { Amount of } \\
\text { organic groups } \\
\left(\mathrm{mmol} \mathrm{g}^{-1}\right)^{c}\end{array}$ \\
\hline MP-LPMSN & 8.0 & 782 & 0.69 & 5.3 & $1.2(1.4)$ \\
\hline CP-LPMSN & 9.3 & 855 & 0.90 & 6.5 & $1.3(1.1)$ \\
\hline AP-LPMSN & 9.3 & 654 & 0.86 & 7.4 & $0.7(0.6)$ \\
\hline AD-LPMSN & 9.9 & 864 & 0.93 & 6.8 & $1.0(-)$ \\
\hline CPD-LPMSN & 8.7 & 898 & 0.74 & 5.3 & $1.0(-)$ \\
\hline
\end{tabular}

${ }_{a}^{a}$ The $\mathrm{d}_{100}$ number represented d-spacing calculated from the PXRD (100) peak. ${ }^{b}$ Specific surface area $\left(\mathrm{S}_{B E T}\right)$, pore volume $\left(\mathrm{V}_{p}\right)$, and mean pore diameter $\left(\mathrm{W}_{B J H}\right)$ were obtained from nitrogen sorption analysis. The specific surface area was measured by BET method. The mean pore diameter was masured by BJH method using adsorption data branch. ${ }^{c .}$ The organic loading was estimated from ${ }^{29} \mathrm{Si}$ solid-state NMR spectra. The number in the parenthesis was calculated from the elemental analysis. 
(a)

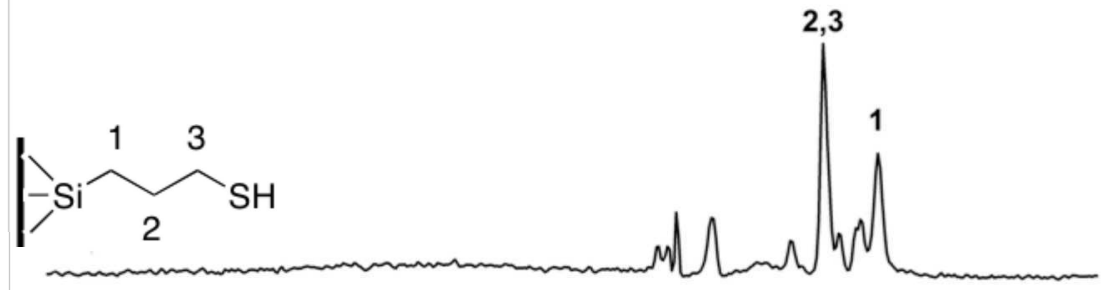

(b)

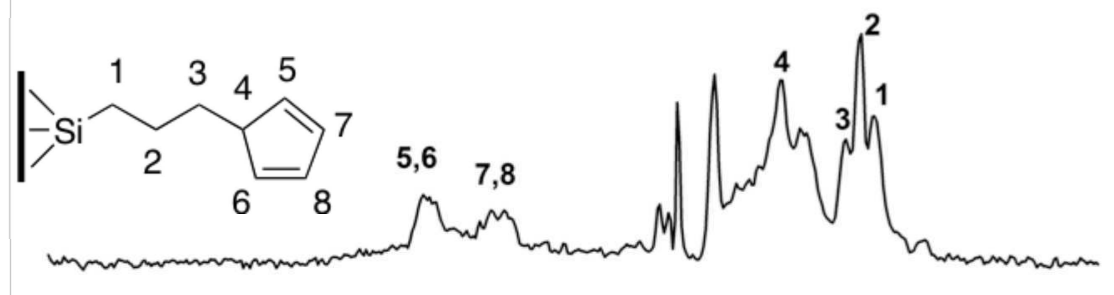

(c)

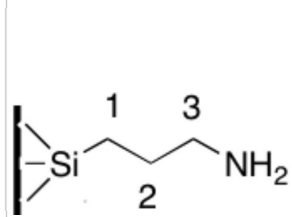

(d)

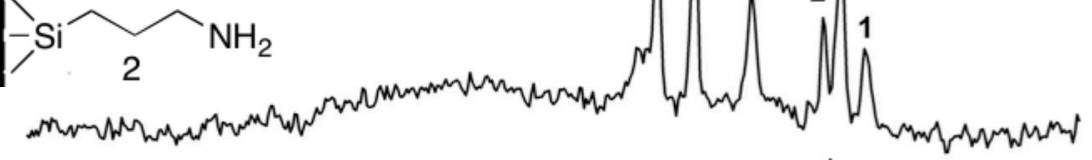

(e)
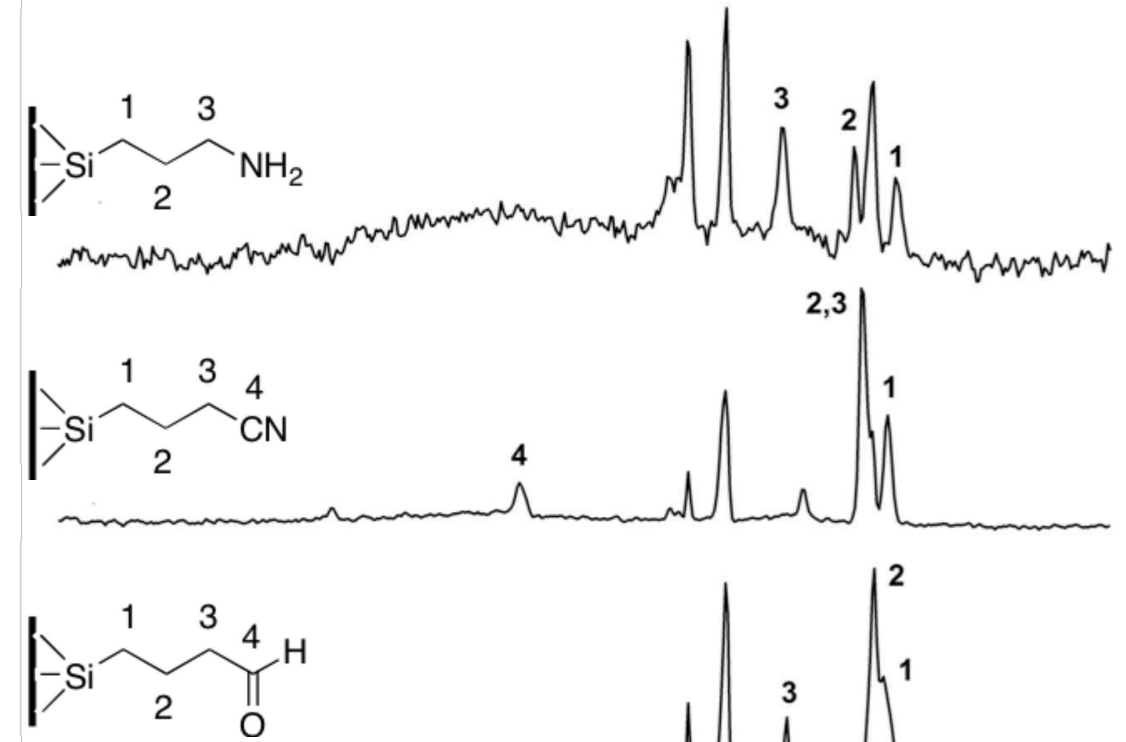

e)

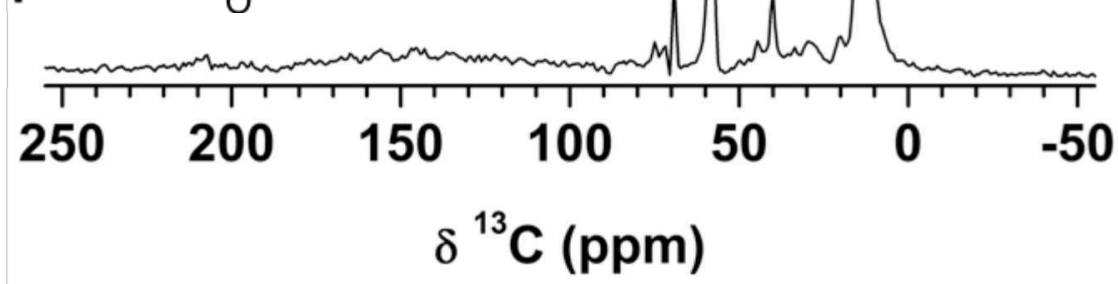

Figure 4-6. ${ }^{13} \mathrm{C}$ solid-state CPMAS NMR spectra of MPLPMSN (a), CPD-LPMSN (b), AP-LPMSN (c), CP-LPMSN (d), and AD-LPMSN (e). 
(a)

(b)

(c)

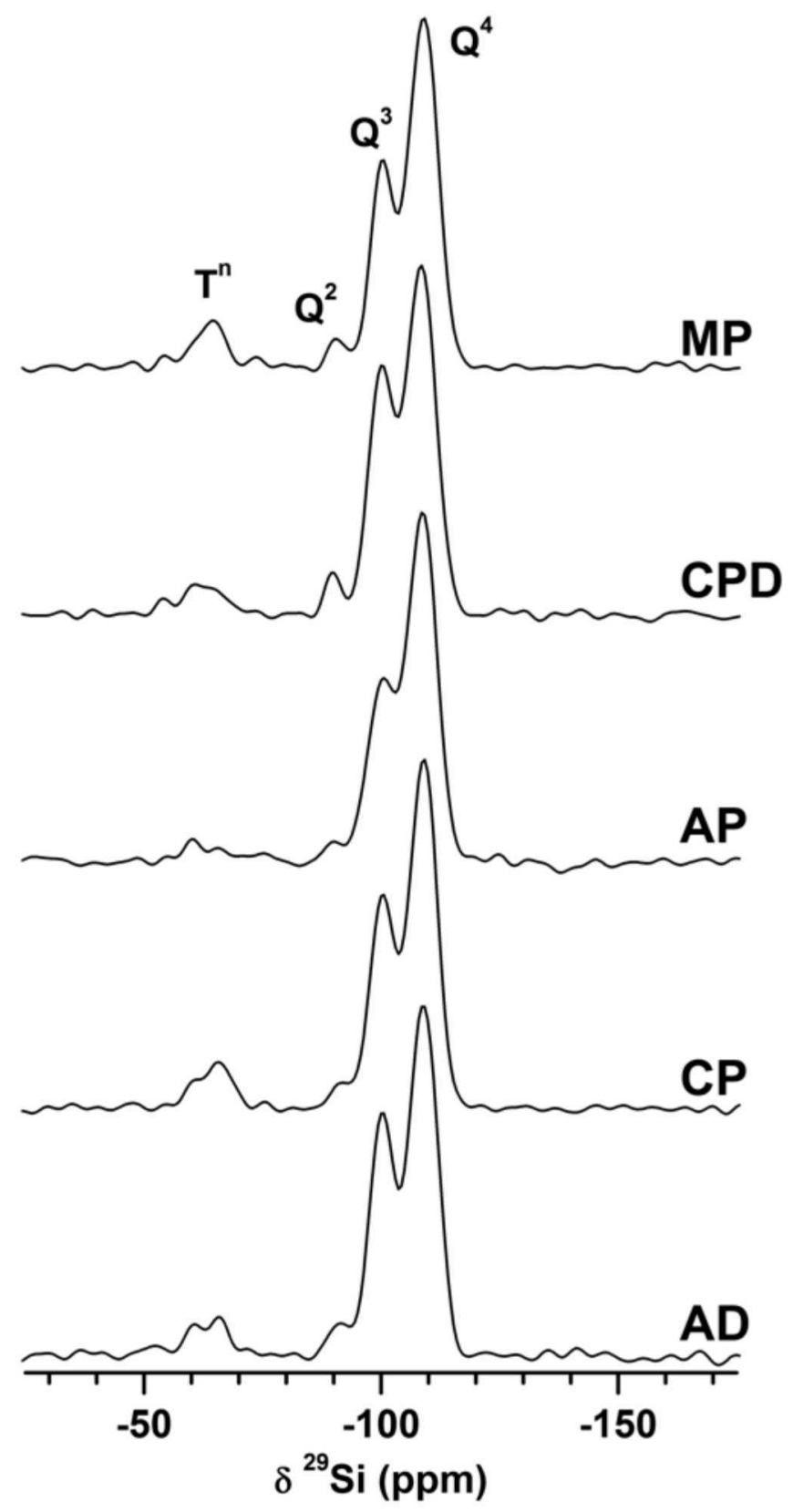

Figure 4-7. ${ }^{29}$ Si DPMAS spectra of MP-LPMSN (a), CPD-LPMSN (b), AP-LPMSN (c), CPLPSMN (d), and AD-LPMSN (e).

Kinetics study of esterification catalyzed by sulfonic acid functionalized mesoporous silica.

To evaluate how the particle size of mesoporous silica supports affect the catalytic activity, the esterification of palmitic acid and methanol was conducted using the aforementioned SALPMSN-100C as the catalyst and compared the reaction result with one catalyzed by sulfonic acid functionalized SBA-15 (SA-SBA15). ${ }^{38}$ The SA-SBA15 material, synthesized according to literature procedures, was composed of a similar mesoporous structure, i.e. 2-D hexagonal channels, and slightly larger pore diameter of 5.6; however, it was determined that these two materials had very different particle sizes and morphology. The SEM image of SA-LPMSN100C displayed individual hexagonal-shaped particles with a particle size similar as its 
precursor, MP-LPMSN, which was around $600 \mathrm{~nm} \times 400 \mathrm{~nm}$ as shown in Figure 4-8. On the other hand, SA-SBA15 exhibited elongated tubular aggregates from primary particles. The length of the mesopores in SA-SBA15 was longer than $1 \mu \mathrm{m}$ with some U-shaped channels.
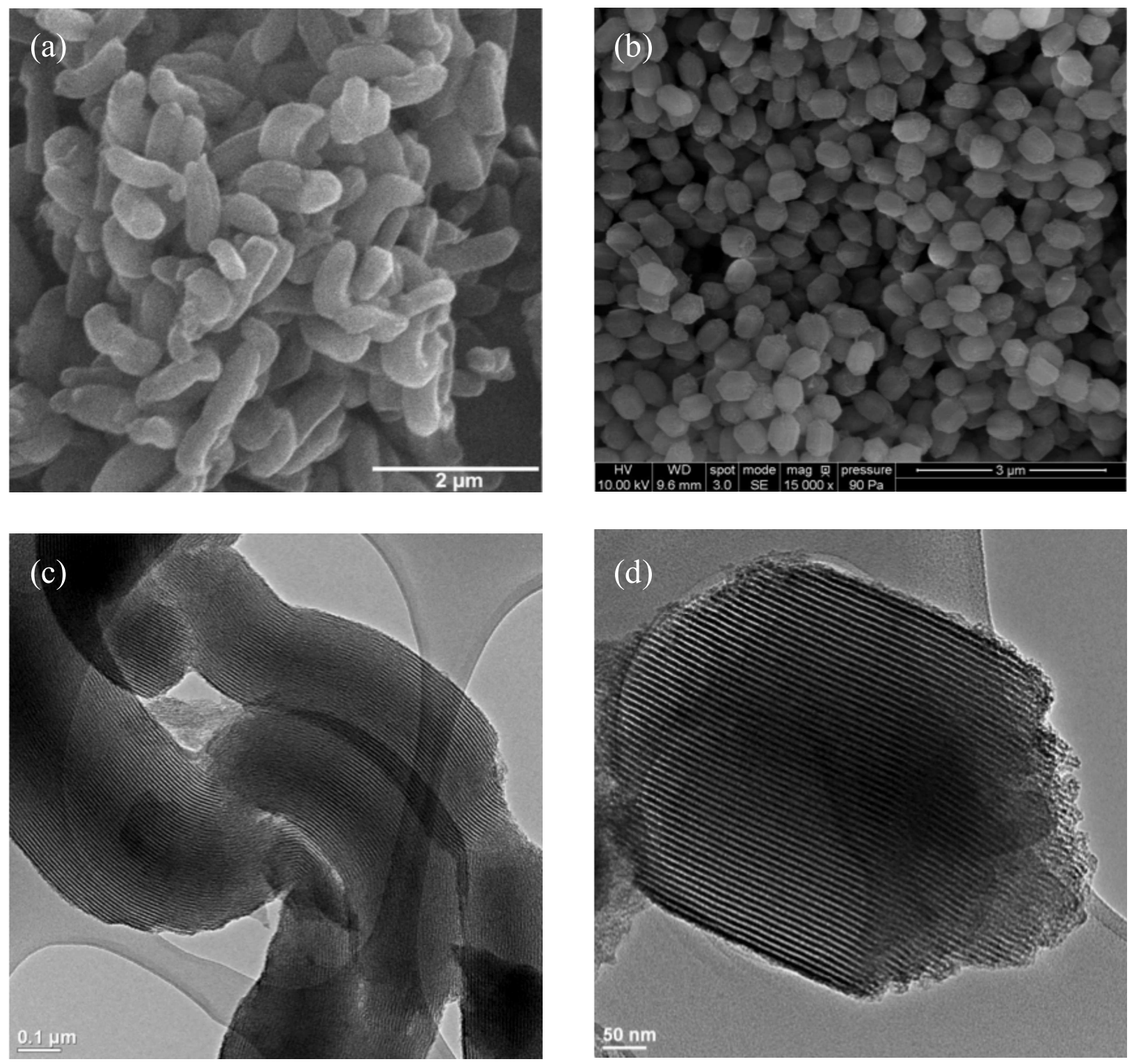

Figure 4-8. SEM images of SA-SBA-15 (a) and SA-LPMSN-100C (b). Scale bar $=2 \mu \mathrm{m}$ and $3 \mu \mathrm{m}$, respectively. TEM images of SA-SBA15 (c) and SA-LPMSN-100C (d). Scale bar $=100 \mathrm{~nm}$ and $50 \mathrm{~nm}$, respectively.

TEM images of two acid functionalized mesoporous silicas unveiled the fine structure of channels with an average depth of $600 \mathrm{~nm}$ for the SA-LPMSN-100C and several micrometers long for the SA-SBA15. The difference in length in the mesopore channels will impact on the mass transport of substrates in and out of channels resulting in dissimilar reaction kinetics. In other words, the reactants, palmitic acid and methanol, have to travel longer distances before reaching the catalytic sites located inside the channels, and the product would also need more time to diffuse from the pores when SA-SBA15 was used as catalyst rather than SA-LPMSN- 
100C. Indeed, as shown in Figure 4-9, the kinetic study showed that the esterification reaction of palmitic acid and methanol catalyzed by SA-LPMSN-100C was much faster $(97 \%$ conversion in $20 \mathrm{~h}$ ) than SA-SBA15. While SA-SBA15 was used as a catalyst, the reaction expressed slow kinetics, which only $89 \%$ of palmitic acid was converted to methyl palmitate at $30 \mathrm{~h}$, even when the reaction was extended to 2 days, the conversion only increased by $0.5 \%$. Such a difference in reaction rate could be attributed to different depths and curvatures of the mesochannels. The short path of pore channels as in the SA-LPMSN-100C offered less diffusion barriers, which results in faster kinetics of reaction and higher reactivity. This kinetic study demonstrated the merits of using these isolated LPMSNs as catalytic supports
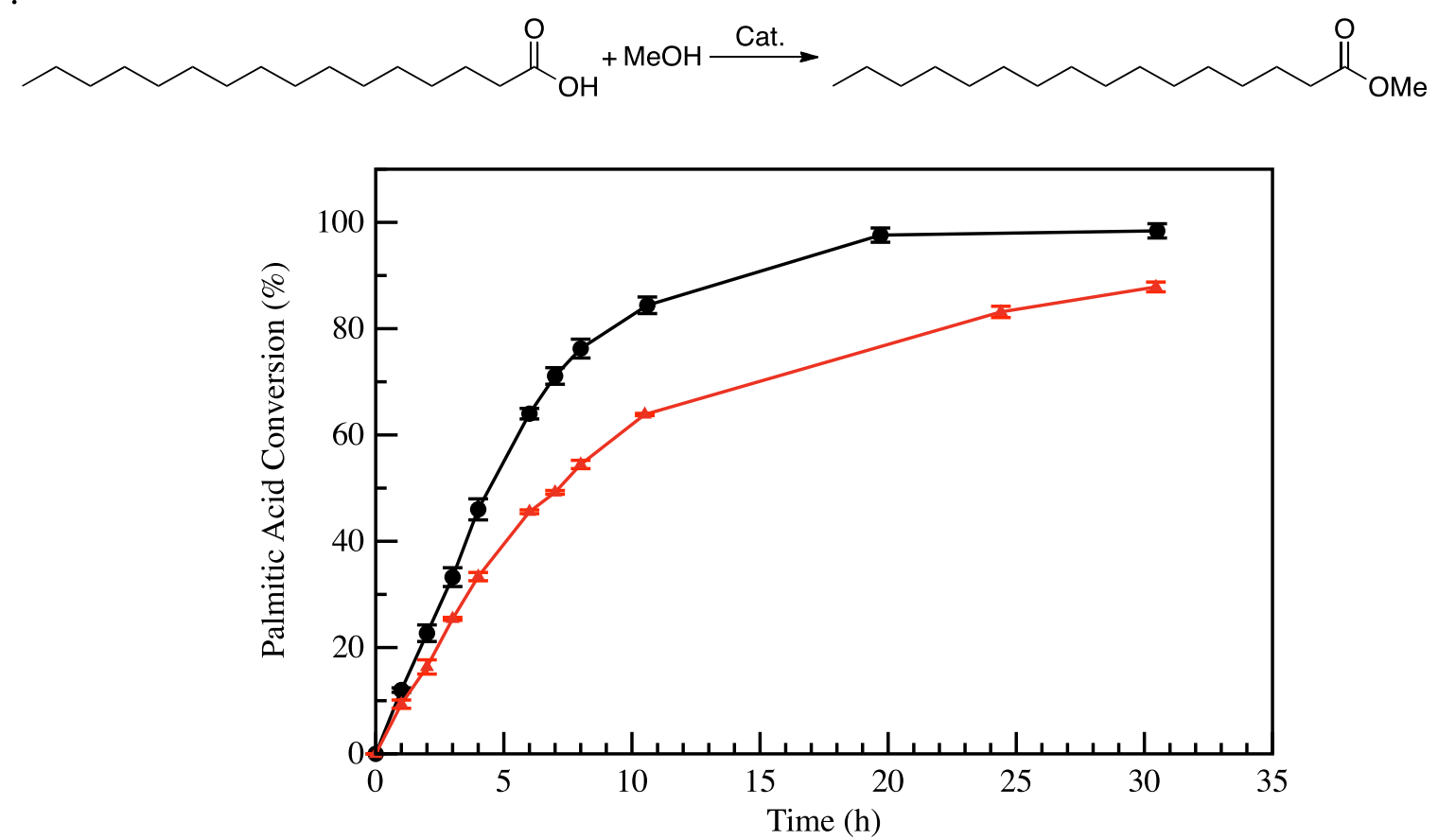

Figure 4-9. Kinetic studies of the esterification reaction catalyzed by SA-LPMSN-100C (black) and SA-SBA15 (red).

\section{Conclusions}

In summary, a synthetic procedure, integrating the synthesis of large-pore mesoporous silica with a co-condensation method, was developed to yield a series of organic functionalized LPMSNs with mono-dispersed particle size, large pore diameter, and chemically accessible surface functional groups. The control of particle size in mesoporous supports has also been demonstrated to be beneficial to catalytic reactivity. We envision that these regular sized wellseparated organic functionalized LPMSNs could serve by its own as a heterogeneous single-site organic catalyst or a superior material support for immobilization of bulky reactive organometallic catalysts.

\section{References:}

1. Kresge, C. T.; Leonowicz, M. E.; Roth, W. J.; Vartuli, J. C.; Beck, J. S., Nature 1992, 359 (6397), 710-712. 
2. $\quad$ Beck, J. S.; Vartuli, J. C.; Roth, W. J.; Leonowicz, M. E.; Kresge, C. T.; Schmitt, K. D.; Chu, C. T. W.; Olson, D. H.; Sheppard, E. W.; Mccullen, S. B.; Higgins, J. B.; Schlenker, J. L., J. Am. Chem. Soc. 1992, 114 (27), 10834-10843.

3. Schacht, S.; Huo, Q.; VoigtMartin, I. G.; Stucky, G. D.; Schuth, F., Science 1996, 273 (5276), 768-771.

4. Huo, Q. S.; Zhao, D. Y.; Feng, J. L.; Weston, K.; Buratto, S. K.; Stucky, G. D.; Schacht, S.; Schuth, F., Adv. Mater. 1997, 9 (12), 974-978.

5. Shio, S.; Kimura, A.; Yamaguchi, M.; Yoshida, K.; Kuroda, K., Chem. Commun. 1998, (22), 2461-2462.

6. Tanev, P. T.; Liang, Y.; Pinnavaia, T. J., J. Am. Chem. Soc. 1997, 119 (37), 8616-8624.

7. Bruinsma, P. J.; Kim, A. Y.; Liu, J.; Baskaran, S., Chem. Mater. 1997, 9 (11), 2507-2512.

8. Yang, S. M.; Yang, H.; Coombs, N.; Sokolov, I.; Kresge, C. T.; Ozin, G. A., Adv. Mater. 1999, $11(1), 52-55$.

9. Trewyn, B. G.; Whitman, C. M.; Lin, V. S. Y., Nano Lett. 2004, 4 (11), 2139-2143.

10. Radu, D. R.; Lai, C. Y.; Wiench, J. W.; Pruski, M.; Lin, V. S. Y., J. Am. Chem. Soc. 2004, $126(6), 1640-1641$.

11. Lai, C. Y.; Trewyn, B. G.; Jeftinija, D. M.; Jeftinija, K.; Xu, S.; Jeftinija, S.; Lin, V. S. Y., J. Am. Chem. Soc. 2003, 125 (15), 4451-4459.

12. Lu, J.; Choi, E.; Tamanoi, F.; Zink, J. I., Small 2008, 4 (4), 421-426.

13. Zhao, Y.; Trewyn, B. G.; Slowing, I. I.; Lin, V. S. Y., J. Am. Chem. Soc. 2009, 131 (24), 8398-8400.

14. Patel, K.; Angelos, S.; Dichtel, W. R.; Coskun, A.; Yang, Y. W.; Zink, J. I.; Stoddart, J. F., J. Am. Chem. Soc. 2008, 130 (8), 2382-2383.

15. Liu, R.; Zhang, Y.; Zhao, X.; Agarwal, A.; Mueller, L. J.; Feng, P. Y., J. Am. Chem. Soc. 2010, 132 (5), 1500-+1501.

16. Patil, Y. B.; Swaminathan, S. K.; Sadhukha, T.; Ma, L. A.; Panyam, J., Biomaterials 2010, 31 (2), 358-365.

17. Chen, H. T.; Huh, S.; Wiench, J. W.; Pruski, M.; Lin, V. S. Y., J. Am. Chem. Soc. 2005, 127 (38), 13305-13311.

18. Huh, S.; Chen, H. T.; Wiench, J. W.; Pruski, M.; Lin, V. S. Y., Angew Chem Int Edit 2005, 44 (12), 1826-1830.

19. Mihalcik, D. J.; Lin, W. B., Angew Chem Int Edit 2008, 47 (33), 6229-6232.

20. Demel, J.; Cejka, J.; Stepnicka, P., J Mol Catal a-Chem 2010, 329 (1-2), 13-20.

21. Melero, J. A.; van Grieken, R.; Morales, G., Chem. Rev. 2006, 106 (9), 3790-3812.

22. Corma, A.; Kan, Q. B.; Navarro, M. T.; PerezPariente, J.; Rey, F., Chem. Mater. 1997, 9 (10), 2123-2126.

23. Sayari, A.; Liu, P.; Kruk, M.; Jaroniec, M., Chem. Mater. 1997, 9 (11), 2499-2506.

24. Slowing, I. I.; Trewyn, B. G.; Lin, V. S. Y., J. Am. Chem. Soc. 2007, 129 (28), 8845-8849.

25. Blin, J. L.; Su, B. L., Langmuir 2002, 18 (13), 5303-5308.

26. Huo, Q. S.; Margolese, D. I.; Stucky, G. D., Chem. Mater. 1996, 8 (5), 1147-1160.

27. Zhao, D.; Feng, J.; Huo, Q.; Melosh, N.; Fredrickson, G. H.; Chmelka, B. F.; Stucky, G. D., Science 1998, 279 (5350), 548-52.

28. Yu, C. Z.; Fan, J.; Tian, B. Z.; Zhao, D. Y.; Stucky, G. D., Adv. Mater. 2002, 14 (23), 1742-1745.

29. Linton, P.; Wennerstrom, H.; Alfredsson, V., PCCP 2010, 12 (15), 3852-3858. 
30. Cauda, V.; Onida, B.; Platschek, B.; Muhlstein, L.; Bein, T., J. Mater. Chem. 2008, 18 (48), 5888-5899.

31. Zhao, D. Y.; Sun, J. Y.; Li, Q. Z.; Stucky, G. D., Chem. Mater. 2000, 12 (2), 275-279.

32. Liu, Y. M.; Cao, Y.; Yi, N.; Feng, W. L.; Dai, W. L.; Yan, S. R.; He, H. Y.; Fan, K. N., J. Catal. 2004, 224 (2), 417-428.

33. Sayari, A.; Yang, Y., Chem. Mater. 2005, 17 (24), 6108-6113.

34. De Witte, K.; Meynen, V.; Mertens, M.; Lebedev, O. I.; Van Tendeloo, G.; SepulvedaEscribano, A.; Rodriguez-Reinoso, F.; Vansant, E. F.; Cool, P., Appl Catal B-Environ 2008, 84 (1-2), 125-132.

35. Meynen, V.; Cool, P.; Vansant, E. F., Microporous Mesoporous Mater. 2009, 125 (3), 170-223.

36. Basaldella, E. I.; Legnoverde, M. S., J. Sol-Gel Sci. Technol. 2010, 56 (2), 191-196.

37. Badley, R. D.; Ford, W. T., J. Org. Chem. 1989, 54 (23), 5437-5443.

38. Margolese, D.; Melero, J. A.; Christiansen, S. C.; Chmelka, B. F.; Stucky, G. D., Chem. Mater. 2000, 12 (8), 2448-2459. 


\section{SUPPORTING INFORMATION OF CHAPTER 4}

Table 4-S1. Tetural properties of MP-LPMSNs prepared in different prehydrolysis time.

\begin{tabular}{|c|c|c|c|c|}
\hline Material $^{a}$ & $d_{100}(n m)$ & $\begin{array}{l}\text { Surface area } \\
\quad\left(\mathrm{m}^{2} / \mathrm{g}\right)^{b}\end{array}$ & $\begin{array}{l}\text { Pore volume } \\
\left(\mathrm{cm}^{3} / \mathrm{g}\right)\end{array}$ & $\begin{array}{l}\text { Mean pore } \\
\text { diameter } \\
(\mathrm{nm})^{c}\end{array}$ \\
\hline MP-LPMSN-0 & 7.8 & 694 & 0.55 & $2 \sim 5.2$ \\
\hline MP-LPMSN-15 & 8.2 & 857 & 0.79 & 4.6 \\
\hline Mp-LPMSN-30 & 8.0 & 782 & 0.69 & 5.3 \\
\hline
\end{tabular}

a. The $x$ in the notation of MP-LPMSN- $x$ indicated the prehydrosis time in min of TMOS before addition of MPTMS. ${ }^{b}$. Specific surface area was calculated from BET method. ${ }^{c}$. Mean pore diameter was calculated based on BJH method.

Table 4-S2. Physical and chemical properties of MP-LPMSN prepared at different aging temperature.

\begin{tabular}{|c|c|c|c|c|}
\hline Material $^{a}$ & $\begin{array}{l}\text { Surface area } \\
\qquad\left(\mathrm{m}^{2} / \mathrm{g}\right)^{b}\end{array}$ & $\begin{array}{l}\text { Mean Pore } \\
\text { diameter }^{(n m)^{c}}\end{array}$ & $\begin{array}{l}\text {-SH loading } \\
(\mathrm{mmol} / \mathrm{g})^{d}\end{array}$ & $\begin{array}{c}\mathrm{H}+\text { amount } \\
(\mathrm{mmol} / \mathrm{g})^{e}\end{array}$ \\
\hline MP-LPMSN-100C & 875 & 4.6 & 1.2 & 0.475 \\
\hline MP-LPMSN-150C & 576 & 6.1 & 1.1 & 0.091 \\
\hline
\end{tabular}

${ }^{a}$. The $y$ in the notation of MP-LPMSN- $y$ indicated the temperature of hydrothermal treatment. ${ }^{b .}$ Specific surface area was calculated from BET method. ${ }^{c}$. Mean pore diameter was calculated based on BJH method. ${ }^{d .}$ Loading of surface thiol group in the MP-LPSMN was estimated from ${ }^{29} \mathrm{Si}$ solid-state NMR spectra. ${ }^{f}$. The acid amount in the sulfonic acid-functionalized LPMSN obtained through oxidation of corresponded MP-LPMSN was measured by acid-base titration using reported method.

Table 4-S3. Structural and chemical properties of SA-LPMSN-100C and SA-SBA15.

\begin{tabular}{|c|c|c|c|c|}
\hline Material & $\begin{array}{l}\text { Surface area } \\
\qquad\left(\mathrm{m}^{2} / \mathrm{g}\right)^{a}\end{array}$ & $\begin{array}{c}\text { Mean Pore } \\
\text { diameter }(\mathrm{nm})\end{array}$ & $\begin{array}{l}\text { Pore channel } \\
\text { length }(\mu \mathrm{m})\end{array}$ & $\begin{array}{l}\text { Loading of sulfonic } \\
\text { acid }(\mu \mathrm{mol} / \mathrm{g})^{c}\end{array}$ \\
\hline $\begin{array}{c}\text { SA-LPMSN- } \\
100 \mathrm{C}\end{array}$ & 838 & 4.8 & $\sim 0.6$ & 375 \\
\hline SA-SBA15 & 669 & 5.6 & $>1$ & 733 \\
\hline
\end{tabular}

${ }^{a}$ Specific surface area was calculated from BET method. ${ }^{b .}$ Mean pore diameter was calculated based on BJH method. ${ }^{c}$. The acid amount in the sulfonic acid-functionalized LPMSN obtained through oxidation of corresponded MP-LPMSN was measured by acid-base titration using reported method. 
(a)

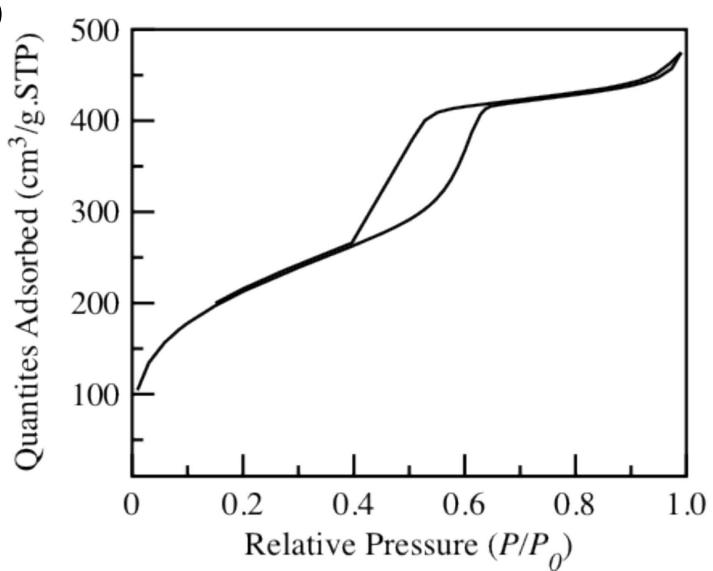

(b)

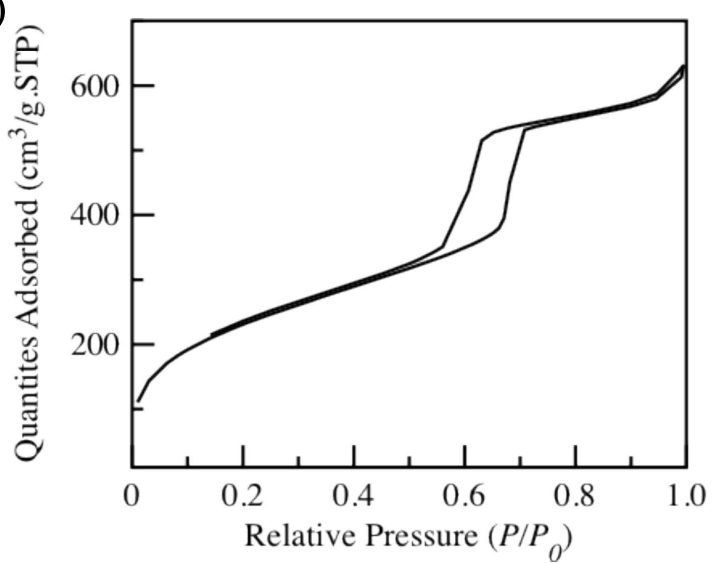

(c)

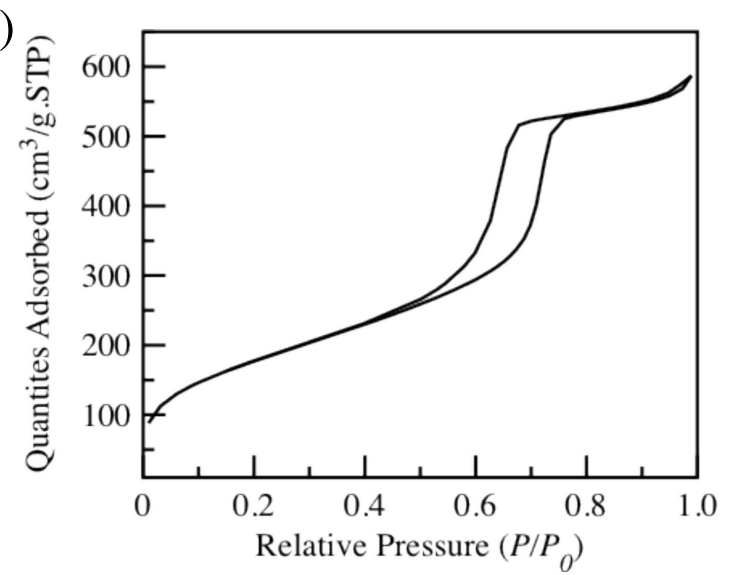

(d)

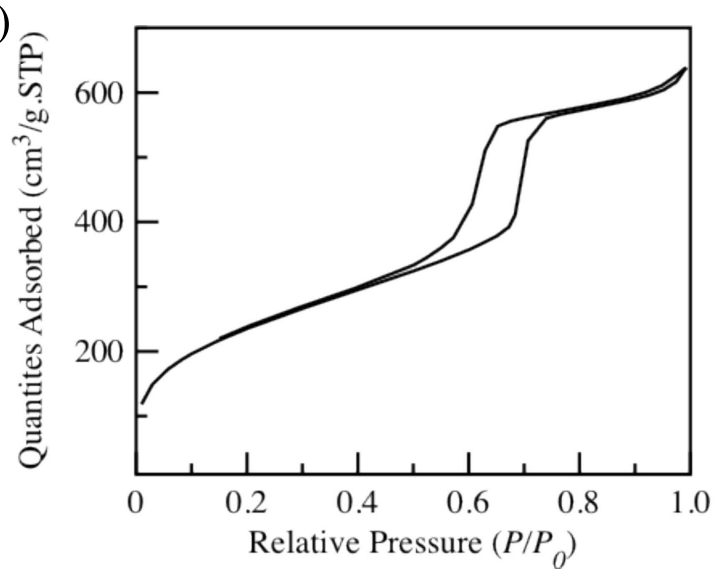

(f)

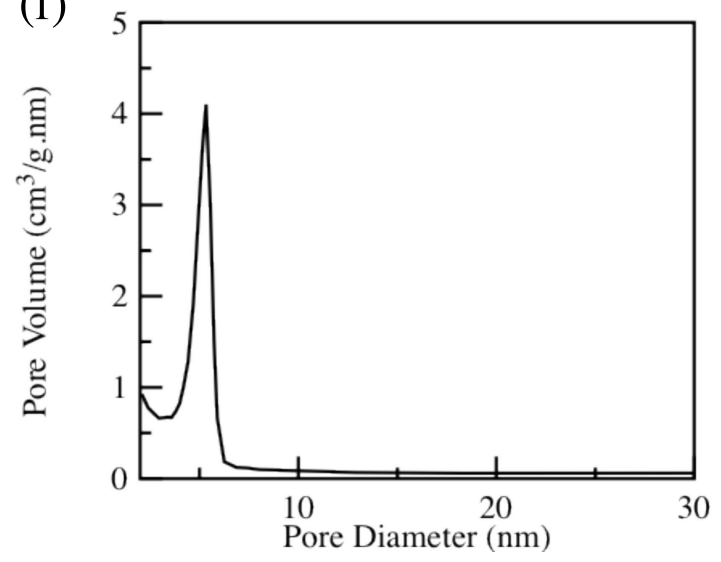

(g)

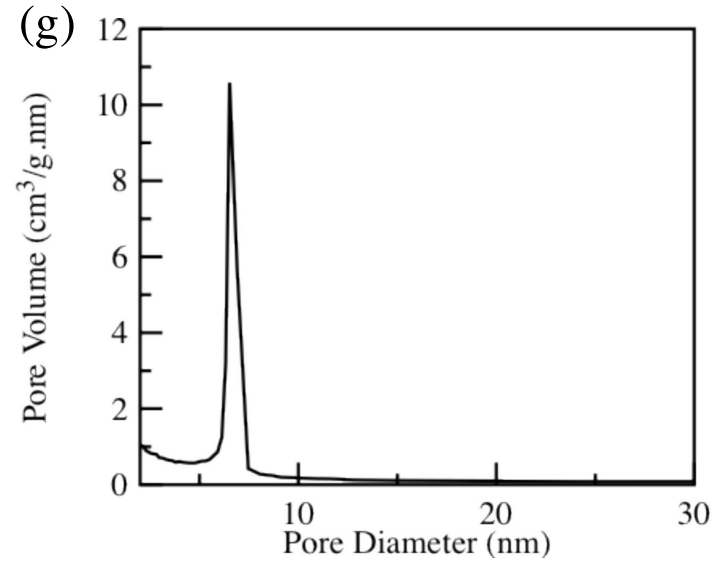

(h)

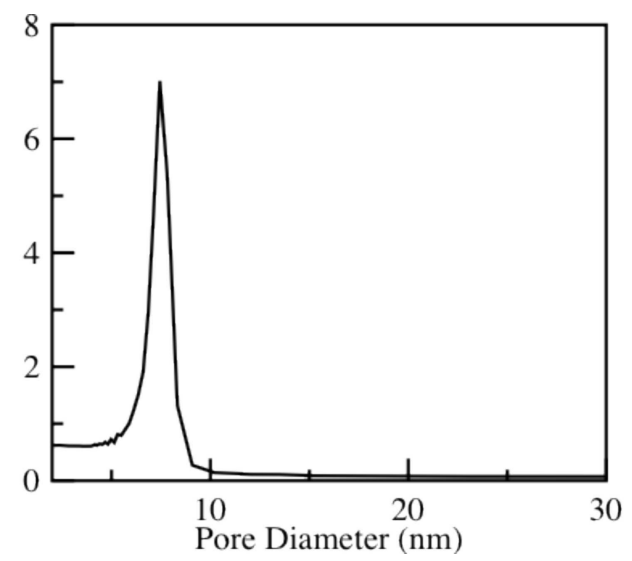

(i)

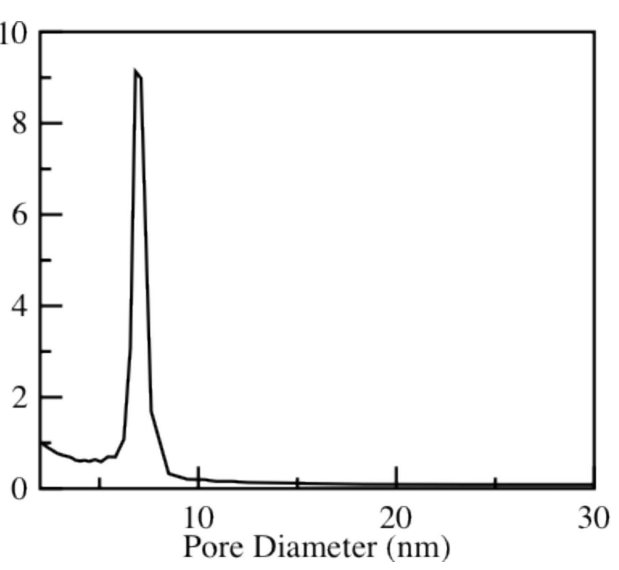


(e)

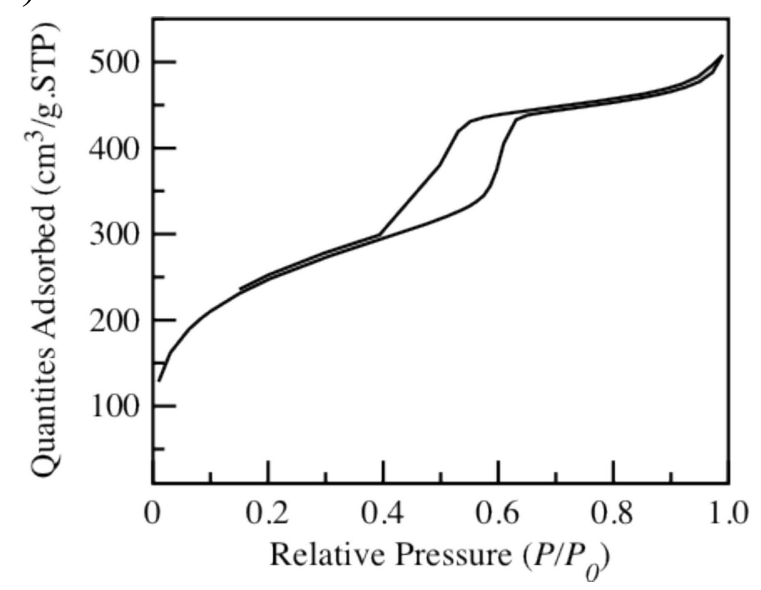

(j)

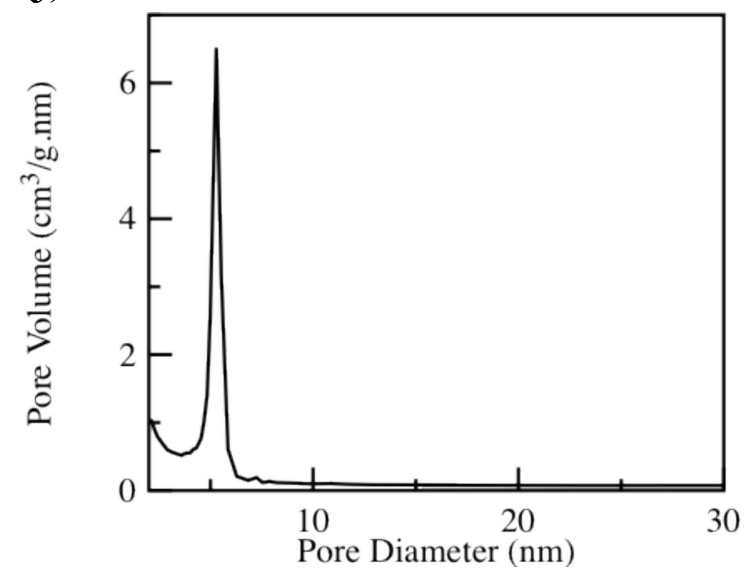

Figure 4-S1. Nitrogen sorption isotherms of MP-LPMSN (a), CP-LPMSN (b), AP-LPMSN (c), AD-LPMSN (d), and CPD-LPMSN (e). BJH pore size distribution of MP-LPMSN (f), CPLPMSN (g), AP-LPMSN (h), AD-LPMSN(i), and CPD-LPMSN (j). 